\title{
A MOdified DETECTOR CONCEPT FOR SUPERCDMS: The HiZIP AND ITS CHARGE PERFORMANCE
}

\author{
by
}

\section{Kedar Mohan Page}

\author{
A thesis submitted to the \\ Department of Physics
}

in conformity with the requirements for

the degree of Master of Science

Queen's University

Kingston, Ontario, Canada

September 2013

Copyright (C) Kedar Mohan Page, 2013 


\section{Statement of Originality}

The projects in this thesis are of collaborative nature. In order to provide a context for the results, and for the sake of completeness, some of the work carried out by collaborators is presented in brief as part of this thesis.

The development of the radioactive $210 \mathrm{~Pb}$ source discussed in chapter 4 and the noise studies described in chapter 5 were carried out by me.

In the G48 data analysis described in chapter 6, I contributed towards development of data quality cuts (section 6.2.1), radial cut efficiency calculations (section 6.2.3) and surface event leakage calculations (section 6.2.4). The data quality cuts that I worked on are: pileup events cut, a cut on standard deviation phonon pre-pulse baseline and a cut to remove saturated phonon pulses. Much of the infrastructure, data quality cuts based on charge information and the scripts used to do the leakage and efficiency calculations already existed. 
In the work discussed in chapter 7, I contributed the most in terms of data taking, development of quality cuts (section 7.2.1), charge calibration (see section 7.2.2) and calculations of radial cut efficiency (see section 7.2.4) and leakage calculation (see section 7.2.7). The data quality cuts that I developed include: pileup event cut, replica events cut, a cut to remove noisy periods and a cut on the standard deviation of pre-pulse phonon baseline as well as the charge threshold cut.

(Kedar Mohan Page )

(September, 2013) 


\section{Abstract}

SuperCDMS (Super Cryogenic Dark Matter Search) is a leading direct dark matter search experiment which uses solid state detectors (Ge crystals) at milliKelvin temperatures to look for nuclear recoils caused by dark matter interactions in the detector. 'Weakly Interacting Massive Particles' (WIMPs) are the most favoured dark matter candidate particles. SuperCDMS, like many other direct dark matter search experiments, primarily looks for WIMPs. The measurement of both the ionization and the lattice vibration (phonon) signals from an interaction in the detector allow it to discriminate against electron recoils which are the main source of background for WIMP detection.

SuperCDMS currently operates about $9 \mathrm{~kg}$ of Ge detectors at the Soudan underground lab in northern Minnesota. In its next phase, SuperCDMS SNOLAB plans to use 100-200 $\mathrm{kg}$ of target mass (Ge) which would allow it to probe more of the interesting and and as of yet unexplored parameter space for WIMPs predicted by theoretical models. The SuperCDMS Queen's Test Facility is a detector test facility which is intended to serve as detector testing and detector research and development purposes for the SuperCDMS experiment.

A modified detector called the 'HiZIP' (Half-iZIP), which is reduced in complex-

ity in comparison to the currently used iZIP (interleaved Z-sensitive Ionization and iii 
Phonon mediated) detectors, is studied in this thesis. The HiZIP detector design also serves to discriminate against background from multiple scatter events occurring close to the surfaces in a single detector. Studies carried out to compare the surface event leakage in the HiZIP detector using limited information from iZIP data taken at SuperCDMS test facility at UC Berkley produce a highly conservative upper limit of 5 out of 10,000 events at $90 \%$ confidence level. This upper limit is the best among many different HiZIP configurations that were investigated and is comparable to the upper limit calculated for an iZIP detector in the same way using the same data. A real HiZIP device operated at the Queen's Test Facility produced an exposure limited $90 \%$ upper limit of about 1 in 100 events for surface event leakage. The data used in these studies contain true nuclear recoil events from cosmogenic and ambient neutrons. This background was not subtracted in the calculation of the upper limits stated above and hence they are highly conservative.

A surface event source was produced by depositing ${ }^{210} \mathrm{~Pb}$ from radon exposure onto a copper plate. This source was then used to take data for a surface event discrimination study of the HiZIP detector operated at the Queen's Test Facility.

A study of the contribution of the noise from capacitive crosstalk between charge sensors in a HiZIP detector configuration was investigated, confirming the expectation that no significant drop in performance compared to an iZIP is to be expected due to this effect. 


\section{Acknowledgements}

I am extremely thankful to Dr.Wolfgang Rau for the opportunity to work on the SuperCDMS experiment under his guidance. It has been a very enriching experience. I am also thankful for his patience and support and for spending great amounts of time to make sure I understood things clearly. He has been a great source of inspiration. The HiZIP project was his idea and I am thankful for the opportunity to work on this project.

I am extremely thankful to the members of the SuperCDMS group at Queen's for a cooperative and conducive environment. Most of the my work discussed in this thesis is the result of a collaborative effort by members of our group. When I joined the group, Joey introduced me to the test facility and the equipment in the test facility and also made me feel at home. Carlos Martinez, Matt Pyle, Jordan Morelli and Arran Phipps helped me a great deal in the study of electronic noise in charge channels. I am also extremely thankful to Scott Hertel for calculating the HiZIP capacitances upon my request.

During the HiZIP analysis on G48, I had the opportunity to work with Yohan Ricci, Carlos Martinez, Oleg Kamev and Chase Crewdson. This was a great learning experience for me. I am also thankful to Bruno Serfass and Philippe De Stefano for their help during this analysis. I am particularly thankful to Yohan for teaching me 
to use CAP, for discussing many aspects of CDMS analysis and often working closely and helping me to troubleshoot problems.

During the G16K analysis I worked closely with Chase Crewdson and I am thankful to him for all his contributions towards making this analysis a success. I am also thankful to Oleg Kamaev for his all his help and his contributions to this project.

I am extremely thankful to Dr.Wolfgang Rau for all his help in the production of the beta source, and in all the projects that I have worked on. I am also thankful to Steve Gillen, Chuck Hearns and Gary Contant for their help on many projects. I am thankful to a number of people in the SuperCDMS collaboration for their support on numerous accounts.

Lastly, but also most importantly, I am thankful to my parents and my friends for their help and support; especially to Sunny and Nam without whose help all this would not have been possible. Special thanks to Alvine and Satoko for their support throughout my degree. 


\section{Glossary}

BLIP Berkley Large Ionization and Phonon mediated

CAP CDMS Analysis Package

CDMS Cryogenic Dark Matter Search

CTF Channel Transfer Function

CMB Cosmic Microwave Background

cQin Radial cut. Removes close to cylindrical wall

DAQ Data Acquisition system

DCRC Digital Control and Readout Card

DIB Detector Interface Board

ER Electron Recoil

FET Field Effect Transistor

HiZip Half iZIP

ICM Intraculster Medium 
iZIP Interleaved Z-sensitive Ionization Phonon mediated

LHC Large Hadron Collider

LSP Lightest Supersymmetric Particle

MISS Multiple Interaction Surface Scatter

MOND Modified Newtonian Dynamics

NR Nuclear Recoil

NSOF Non Stationary Optimal Filter

NTD Neutron Transmutation-Doped

OF Optimal Filter

oZIP Original Z-sensitive Ionization Phonon mediated detector

QET Quasiparticle-trap-assisted Electrothermal-feedback

QTF Queen's Test Facility

SNOLAB Sudbury Neutrino Observatory Lab

SQUET A combined module of SQUID and FET cards

SQUID Superconducting Quantum Interference Device

SSDS Sloan Digital Sky Survey

SUF Stanford Underground Facility

SUSY Supersymmetry 
TES Transition Edge Sensor

USEL Uncorrected Surface Event Leakage

WIMP Weakly Interacting Massive Particle

WMAP Wilkinson Microwave Anisotropy

XTF Crosstalk Transfer Function

ZIP Z-sensitive Ionization Phonon mediated 


\section{Table of Contents}

Statement of Originality

Abstract

\begin{tabular}{|ll}
\hline Acknowledgements & v
\end{tabular}

Glossary vii

Table of Contents $\quad \mathrm{x}$

List of Tables $\quad$ xii

List of Figures $\quad$ xiii

Chapter 1:

Introduction ............... 1

1.1 The case for dark matter in the universe . . . . . . . . . . . . . . 1

1.2 A few plausible solutions to the dark matter problem . . . . . . . . . 8

1.3 Search for particle dark matter . . . . . . . . . . . . . . . . . . . . 12

Chapter 2:

The CDMS Experiment . . . . . . . . . . . 21

2.1 Introduction . . . . . . . . . . . . . . . . . . . . 21

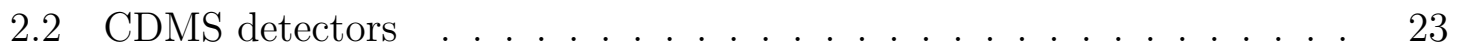

2.3 Backgrounds . . . . . . . . . . . . . . . . . . . . . . . . . . . . 34

\section{Chapter 3:}

Queen's Test Facility ............ 37

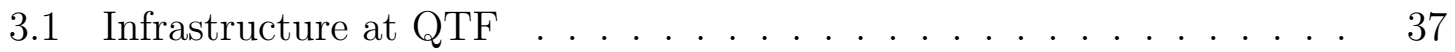

3.2 Data processing and Analysis tools . . . . . . . . . . . . . . . . . 45

3.3 Noise environment . . . . . . . . . . . . . . . . . . . . . . . . . . . . . . . . . . . . . . .

3.4 Detector de-neutralization . . . . . . . . . . . . . . . 46 
Chapter 4:

Fabrication of a ${ }^{210} \mathrm{~Pb}$ Beta Source $\ldots \ldots \ldots \ldots \ldots$

4.1 Motivation . . . . . . . . . . . . . . . . . . . . 50

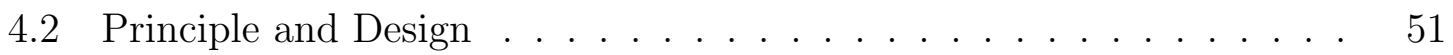

4.3 Experimental setup and Procedure $\ldots \ldots \ldots \ldots \ldots$

Chapter 5:

The HiZIP and Charge Noise Studies $\ldots \ldots \ldots$. . . . 59

5.1 The HiZIP: concept and motivation $\ldots \ldots \ldots \ldots \ldots$

5.2 Electronic noise in charge readout circuit . . . . . . . . . . . . . 64

5.3 HiZIP viability studies using G48 iZIP data. . . . . . . . . . . 77

5.4 The G16K HizIP. $\ldots \ldots \ldots \ldots \ldots \ldots$

Chapter 6:

G48 HiZIP Analysis . . . . . . . . . . . . . . . 82

$6.1 \quad$ Analysis using sum based optimal-filter quantities . . . . . . . . . 83

6.2 Full G48 HiZIP analysis with HiZIP data quality selection. . . . . . . 88

\section{Chapter 7:}

G16K HiZIP $\ldots \ldots \ldots \ldots \ldots \ldots$. . . . . . . . . . . . . .

7.1 Operating the G16K detector at SuperCDMS Queen's Test Facility . 111

$7.2 \quad$ G16K Data analysis . . . . . . . . . . . . . . . . . . . . . . . 115

Chapter 8:

Conclusion $\ldots \ldots \ldots \ldots \ldots \ldots \ldots$

Bibliography . . . . . . . . . . . . . . . . . . . . 142

\section{Appendix A:}

Noise Calculations . . . . . . . . . . . . 153

A.1 Function Definitions . . . . . . . . . . . . . . . . . . . 153

A.2 Derivation of $\mathrm{Q}_{i} \mathrm{CTF}$ and intrinsic channel noise $\ldots \ldots \ldots \ldots . . .154$

A.3 Crosstalk from $\mathrm{Q}_{o}$ noise sources in $\mathrm{Q}_{i} \ldots \ldots \ldots \ldots \ldots$

A.4 Component values and constants . . . . . . . . . . . . . . 162 


\section{List of Tables}

6.1 Uncorrected surface event leakage estimates for various HiZIP config-

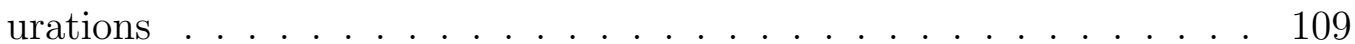

7.1 Compares uncorrected surface event leakage estimates for the G16K and the G48 HiZIP detectors . . . . . . . . . . . . . . . . . . . . . . . 135 


\section{List of Figures}

1.1 Rotation curve for Andromeda galaxy . . . . . . . . . . . . . . . . . 5

1.2 Composite image of the Bullet Cluster . . . . . . . . . . . . . . . . . 9

1.3 Results and projected limits for spin-independent WIMP-nucleon interactions from direct dark matter searches . . . . . . . . . . . . . . . 19

2.1 Ionization versus recoil energy in a ZIP detector . . . . . . . . . . . . 24

2.2 Yield versus recoil energy in a ZIP detector . . . . . . . . . . . . . . . 25

2.3 CDMS ZIP detector $\ldots \ldots \ldots \ldots$. . . . . . . . . . . . . . . . 26

2.4 The iZIP detector . . . . . . . . . . . . . . . . . . . . . . . . 28

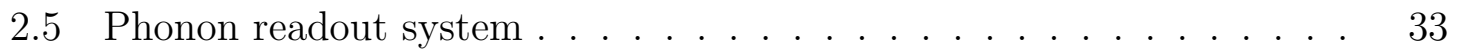

2.6 CDMS II active and passive shielding . . . . . . . . . . . . . . . . . 35

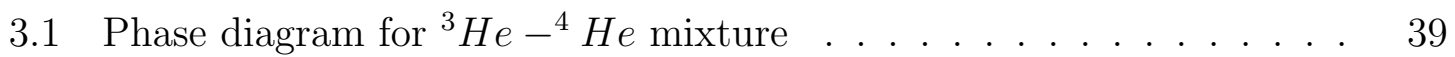

3.2 Cryostat temperature stages . . . . . . . . . . . . . . . . . . . 40

3.3 Components of the tower . . . . . . . . . . . . . . . . . . . . . . 42

3.4 DCRC board . . . . . . . . . . . . . . . . . . . . . . . 44

3.5 Noise spectral density . . . . . . . . . . . . . . . . . . 46

$3.6 \quad$ Black aluminium sheet applied on the inside of the $600 \mathrm{mK}$ can . . . 48

4.1 Radon decay chain . . . . . . . . . . . . . . . . . . . . . . . . 51

4.2 Radon source from Pylon (Model RN-1025) . . . . . . . . . . . . . . 52

4.3 Exposure chamber schematic . . . . . . . . . . . . . . . 55

4.4 Schematic of radon exposure of copper plate . . . . . . . . . . . . . . 56

4.5 Exposure chamber connected to HV supply . . . . . . . . . . . . . . . 57

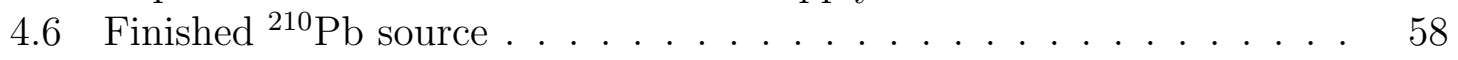

$5.1 \quad$ A MISS event in an iZIP detector caused by a double scatter event . 61

5.2 Sensor layout for HiZIP and iZIP detectors . . . . . . . . . . . . . . 62

$5.3 \quad$ HiZIP wiring configurations $\ldots \ldots \ldots$. . . . . . . . . . 63

5.4 Charge readout circuit for SuperCDMS detectors . . . . . . . . . . 65

5.5 Charge readout circuits of two capacitively coupled charge sensors. . . 66

5.6 Coupled $\mathrm{Q}_{\text {inner }}-\mathrm{Q}_{\text {outer }}$ charge readout circuit . . . . . . . . . . . . 68

5.7 Equivalent circuit used to calculate $\mathrm{Z}_{Q \circ} . \ldots \ldots$. . . . . . . . 68 
$5.8 \mathrm{Q}_{i}$ channel and crosstalk transfer functions for iZIP. . . . . . . . . . . 70

$\begin{array}{llll}5.9 & Q_{p} \text { channel and crosstalk transfer functions for HiZIP configuration } 1 . & 71\end{array}$

$\begin{array}{lll}5.10 & \mathrm{Q}_{p} \text { channel and crosstalk transfer functions for HiZIP configuration } 2 . & 72\end{array}$

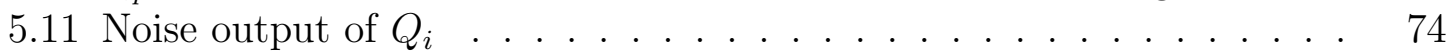

5.12 Crosstalk noise at the output of $Q_{i} \ldots \ldots$. . . . . . . . . . . . 75

5.13 Total noise at the output of $Q_{i} \ldots \ldots$. . . . . . . . . . . . . 75

5.14 Noise at the output of $Q_{p}$ in HiZIP configuration $1 \ldots$. . . . . . . . . 76

5.15 Noise at the output of $Q_{p}$ in HiZIP configuration 2 . . . . . . . . . . 76

5.16 Comparison on total noise for the output of the charge amplifier . . . 77

5.17 The G16K HiZIP charge sensor wiring scheme in the G16K naming

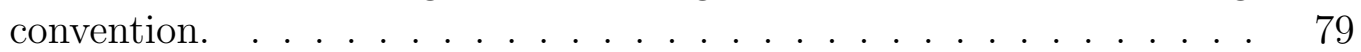

5.18 Performance of a new charge channel $\left(Q_{p}\right)$. . . . . . . . . . . . . 80

5.19 A plot of $Q_{p}$ vs. $Q_{1}$ coloured by $Q_{2} \ldots \ldots$. . . . . . . . . . 81

6.1 Yield plot for G48 iZIP detector . . . . . . . . . . . . . . . . . . . . . 85

6.2 Yield vs. recoil energy for phonon sensors on Side 1 . . . . . . . . . . 87

6.3 Yield vs. recoil energy for phonon sensors on Side 2 . . . . . . . . . . 87

6.4 Example of phonon $\chi^{2}$ cut: . . . . . . . . . . . . . . . . 90

6.5 Examples of pileup events . . . . . . . . . . . . . . . . . . 91

6.6 Mean of the end phonon trace vs. recoil energy and slope of distribution subtracted to simplify cut definition . . . . . . . . . . . . . . 92

6.7 Summed phonon pulses of events at the cut boundary . . . . . . . . . 93

6.8 Phonon traces of a 'Glitch' event . . . . . . . . . . . . . . . . . . . . 95

6.9 Glitch event cut . . . . . . . . . . . . . . . . . . . 96

6.10 Charge symmetry cut for G48 analysis . . . . . . . . . . . . . . . . . 97

6.11 Example of side 1 radial cut in G48 analysis . . . . . . . . . . . . . . 98

6.12 Yield vs. recoil energy for the HiZIP with phonon sensor on side 2 . . 99

6.13 Fiducial volume of iZIP detector . . . . . . . . . . . . . . . . . . . . . 101

6.14 Efficiency losses for radial cut on side 1 for HiZIP compared to iZIP in the NR band . . . . . . . . . . . . . . . . . . . . 103

6.15 Efficiency losses for radial cut on side 2 for HiZIP compared to iZIP in the NR band . . . . . . . . . . . . . . . . . . . . . . . . . . 104

6.16 Efficiency losses for radial cut on side 1 for HiZIP compared to iZIP in the ER band . . . . . . . . . . . . . . . . . . . . . . . . . . 105

6.17 Efficiency losses for radial cut on side 2 for HiZIP compared to iZIP in the ER band . . . . . . . . . . . . . . . . . . . . . . 106

6.18 Leakage of beta events passing the charge symmetry cut . . . . . . 110

7.1 Schematic of the G16K detector . . . . . . . . . . . . . . . . . . . . 112

7.2 Data acquisition setup . . . . . . . . . . . . . . . . . . . 114

7.3 Charge $\chi^{2}$ cut on the $\mathrm{Q}_{2}$ charge channel . . . . . . . . . . . . . 117 
7.4 Algorithm to identify and discard pileup events . . . . . . . . . . . . 118

7.5 Passage fraction of pileup cut . . . . . . . . . . . . . . . . . . 119

7.6 Example of two consecutive events being replicas . . . . . . . . . . . 120

7.7 Histogram of phonon pre-pulse baseline standard deviation for channel

$\mathrm{PA} \ldots \ldots \ldots \ldots \ldots 122$

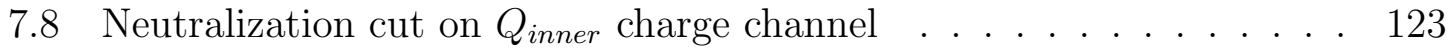

7.9 Example of bad periods for uncalibrated charge OF quantity of charge channel $Q_{2}$. . . . . . . . . . . . . . . . . . . . . . . . . . . . . . . . . 124

$7.10 Q_{\text {inner }}$ and $Q_{\text {outer }}$ calibration . . . . . . . . . . . . . . 125

7.11 Uncalibrated and calibrated charge spectra of channel $Q_{2}$. . . . . . . 126

7.12 Late integral distributions of channel PA vs PB for a sample of good

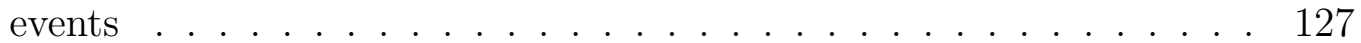

7.13 Phonon traces of an event before and after relative calibration. . . . . 128

7.14 G16K charge symmetry cut . . . . . . . . . . . . . . . . . 130

7.15 G16K radial cut . . . . . . . . . . . . . . . . . . . . . 131

7.16 Radial cut efficiencies for bulk ER and NR events . . . . . . . . . . . 132

7.17 G16K yield bands . . . . . . . . . . . . . . . . . . . . 133

7.18 G16K charge threshold cut . . . . . . . . . . . . . . . . . . . . . . . . 134

7.19 G16K surface event discrimination . . . . . . . . . . . . . . . . . . . 137

7.20 G48 surface event discrimination with hardware and bias configuration of $\mathrm{G16K} \ldots \ldots \ldots \ldots \ldots 138$ 


\section{Chapter 1}

\section{Introduction}

\subsection{The case for dark matter in the universe}

Astronomical data over several decades point to the existence of a new kind of matter which is not part of the standard model of particle physics; a kind of matter that does not shine when light falls on it, termed "Dark Matter". Dark matter seems to hold the components of galaxies and galaxy clusters together and makes up about $23 \%$ of the total energy content of the universe [1] 3]. While many instances of the gravitational effects of dark matter have been observed so far, efforts to either directly detect it in terrestrial detectors or indirectly detect it by looking for signals from dark matter annihilation have not yielded any confirmed signal. Thus, dark matter of the universe continues to remain elusive and enigmatic. SuperCDMS is a direct dark matter search experiment which uses cryogenic detectors to look for WIMP interactions. Due to a dominant background from particles in cosmic rays at the surface of the earth, these experiments are performed deep underground. They also try to minimize radioactivity in and around the detectors. The next generation of the SuperCDMS experiment will 
be at SNOLAB in Sudbury (Ontario) which is much deeper than its present site at the Soudan underground lab (Minnesota) and will further reduce the cosmogenic background. The bulk of this thesis describes R\&D work carried out towards a simplified detector with additional background suppression. In this chapter, we will briefly review some key evidence for the existence of dark matter, some candidates for what dark matter could be and finally discuss some prominent experimental efforts to find dark matter with a focus on direct searches. In chapter 2 we will discuss the SuperCDMS experiment in some detail and provide a context for the work carried out here. In chapter 3 we will discuss the SuperCDMS detector test facility at Queen's and explain some general aspects of the experimental setup used in this work which form the infrastructure of this facility. Chapter 4 will discuss the production of a radioactive source which is used for studying detector performance. Chapter 5 introduces a modified detector design called the 'HiZIP' and makes an assessment of the effect of electronic noise in charge signal readout of a HiZIP detector. Chapter 6 discusses studies carried out to assess the performance expected from a HiZIP detector. In the end, chapter 7 discusses an experiment using a real HiZIP detector which was operated at the Queen's Test Facility and compares its performance to that expected from the studies described in chapter 6 .

\subsubsection{Galaxy clusters}

\section{Virial measurements}

The first evidence for the existence of dark matter dates back to observations of the coma cluster by Fritz Zwicky in the 1930s [4] [5]. These measurements and similar ones by Smith on the Virgo cluster [6] used the virial theorem to estimate the dynamical 
mass of the clusters and then made a comparison to the expected mass from the observed luminosity using typical mass to light ratios known at that time. They found that the mass required to account for the observed velocity dispersion of the galaxies in the cluster was about two orders of magnitude higher than estimated from the typical mass to light ratios [5] [6]. This could alternatively be explained without invoking exotic mechanisms by simply postulating that there could be extra material that is not visible and not accounted for in the mass to light ratios calculated for galaxies.

\section{X-ray emissions}

X-ray emissions of hot ionized gas in hydrostatic equilibrium in the gravitational potential of galaxy clusters can be used to deduce the cluster mass. The estimates of cluster mass from these observations agree with the virial measurements described above. However the measurements were oblivious of the existence of such gas, which in fact dominates the cluster mass, but even the amount of gas found leaves a factor of 10 to be explained [7] [8].

\subsubsection{Galaxy rotation curves}

\section{Optical measurements}

There was not much progress in dark matter research until the 1970s when it was reinvigorated by Vera Rubin and her colleagues' observations of rotation curves of spiral galaxies [9]. The rotation curves from Rubin and Ford's measurements are plots of rotational velocities of gaseous matter and stars around the galaxy as a function of radial distance from the galaxy centre. These measurements used specific optical 
lines from the hydrogen electronic transition spectrum from a wide range of spatially distributed gaseous regions in the galactic disc and beyond [9]. The rotational velocity in simple Newtonian circular motion with gravitation as the centripetal force is given as $v(r)=\sqrt{G M(r) / r}$ where $G$ is Newton's gravitational constant and $M(r)$ is the mass enclosed in a sphere of radius $r$. Hence, the rotational velocities would be expected to fall off as $1 / \sqrt{r}$ beyond all the visible matter. The rotational velocity profile with distance from the galactic centre for M31 (Andromeda galaxy) is shown in figure 1.1. It is clear that the rotational velocities do not follow the $1 / \sqrt{r}$ fall off with radial distance as expected from Newtonian or Keplerian dynamics but rather tend to level out slightly below the observed maximum. Such a rotational velocity profile can be explained assuming a dark matter halo which extends radially well beyond the observable matter in these galaxies. A dark matter halo that extends well beyond observable matter in which the mass enclosed in a sphere of radius $r$ is linearly proportional to $\mathrm{r}$ could make the $v(r)$ profile for observable matter stay flat at high radii. 


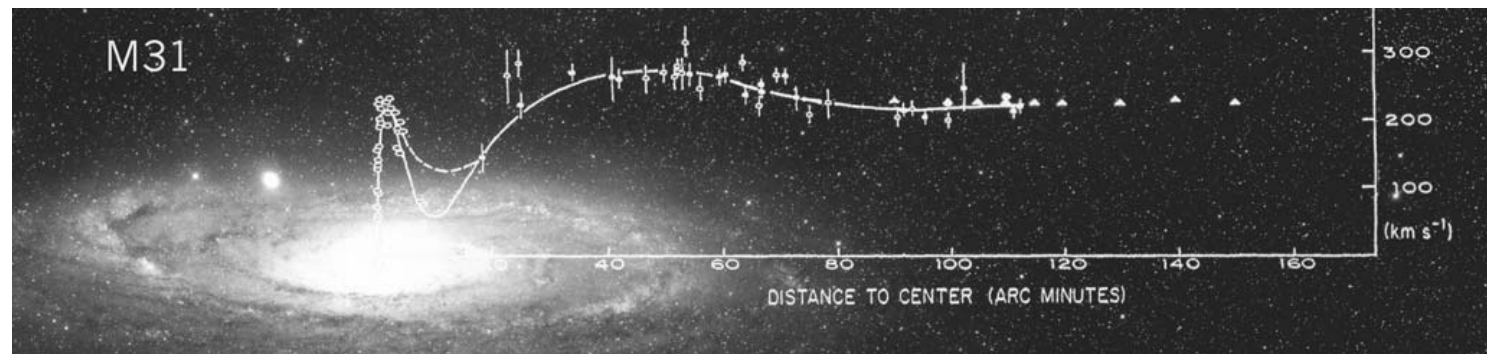

Figure 1.1: Rotation curve for M31 (Andromeda galaxy) superimposed on an M31 image from Palomar sky survey (Figure from [10]). One arc minute corresponds to about 0.74 light years. Circular markers are from optical measurements and triangular markers (90 to 150 arc minutes) are from radio astronomy measurements.

\section{Radio astronomy measurements}

The $21 \mathrm{~cm}$ emission in neutral hydrogen comes from the transition of spin aligned (higher energy) to spin non-aligned states of the hydrogen atom with the electron in its lowest orbital. Although this transition has low probability, it can be observed due to the abundance of hydrogen. Radio astronomy measurements of the $21 \mathrm{~cm}$ line from neutral hydrogen in spiral galaxies confirms the rotational velocity profile seen in optical measurements.

\subsubsection{Gravitational lensing}

Gravitational lensing is a natural consequence of Einstein's general relativity where light passing by massive objects is deflected due to the effect of the mass on the geometry of the surrounding space-time. Galaxy clusters can create significant distortion 
and multiple images of sources behind them. Galaxy clusters are the largest known collection of gravitationally bound entities in the universe. Strong distortion caused by them is usually referred to as strong gravitational lensing. Although strong lensing is a very useful technique to measure cluster mass and matter density profile, it is intricate and involved and is not suitable to be used to study large statistical samples from wide-field surveys.

Another technique called weak lensing refers to measurements using small distortions in ellipticity and orientation of a background galaxy's image when its light passes by a foreground cluster. Although weak lensing does not provide a significant amount of information from the measurement of an individual background galaxy, averaging over a large ensemble of sources (single galaxies) makes it possible to deduce the density profile of the foreground cluster. Weak lensing was applied to about 28 million galaxy images and 130,000 galaxy clusters from SDSS (Sloan Digital Sky Survey) to estimate cluster masses and then make a comparison to the light to mass ratios [11. These studies again reveal a 21-28 \% contribution to the universe's critical density from matter in the universe and point to the existence of significant amounts of dark matter in galaxy clusters [11] [12].

\subsubsection{The cosmic microwave background}

The cosmic microwave background (CMB) is among the most significant discoveries in cosmology in the last century. The $\Lambda$-CDM model has now come to be called standard cosmology, the most successful model of the universe and most of its history. It uses a combination of ordinary matter (standard model of particle physics), Cold Dark Matter (CDM) which is non-relativistic in the early universe, and dark energy which 
sets the value of the cosmological constant $\Lambda$ [13]. At a certain point in early history, the universe was full of hot plasma and radiation in thermal equilibrium. After the temperature dropped enough for atoms to form, the universe became transparent to electromagnetic radiation. This thermal radiation red shifted with the expansion of the universe and is now observed as a very nearly uniform glow in all directions. The spectrum of this radiation matches very well with a black body of $2.73 \mathrm{~K}$ and has a peak in the microwave range.

A detailed all-sky map of small anisotropies in the CMB temperature measured to $\mu \mathrm{K}$ accuracy was made using the data from WMAP (Wilkinson Microwave Anisotropy Probe). The angular power spectrum of these temperature differences tells us the power stored in small scale and large scale fluctuations in the CMB temperature. Large angular scales correspond to smaller multipole moments and vice versa. The relative and absolute amplitudes of the first few peaks in this power spectrum are highly sensitive to the baryonic and non-baryonic matter density in the early universe [13. The best fit cosmological model to explain the power spectrum is called the minimal $\Lambda$-CDM model which requires $\sim 23 \%$ of non relativistic non-baryonic matter in the early universe [3].

\subsubsection{Primordial light element abundances}

Low metallicity regions in the universe indicate lack of star formation in that region. Absorption of quasar light by ionized hydrogen gas in low metallicity regions provides a good way to measure the relative primordial abundances of light elements. In particular, Lyman- $\alpha$ absorption spectra provide a reliable estimate of the total deuterium to hydrogen $(\mathrm{D} / \mathrm{H})$ abundance in the universe. The relative primordial abundances 
of light elements significantly constrain the baryonic matter density to $4.5 \%$ of the critical density of the universe which is clearly too low to form the dominant matter component of the universe as established from other studies [14].

\subsubsection{Bullet Cluster}

A prime example of dark matter in galaxy clusters is the 'Bullet Cluster'. The Bullet Cluster (see figure 1.2) has two clusters whose galaxies and dark matter halos seem to have passed through each other relatively intact while intergalactic gas is lagging behind due to drag forces 15. The hot intergalactic gas emits X-rays and is shown in false colour pink in figure 1.2, while the matter density calculated using weak-lensing is shown in false colour blue. The Bullet Cluster makes a compelling case for the existence of dark matter.

\subsection{A few plausible solutions to the dark matter problem}

\subsubsection{MOND}

A different approach to solve the dark matter problem is to modify the theory of gravity to match the observations. MOND (Modified Newtonian Dynamics) is one such phenomenological approach which explains the rotation curves in spiral galaxies [16]. Efforts have been made to construct relativistic theories which reduce to MOND in the classical limit [17]. However, these theories do not explain effects seen on scales larger than galaxies, for example the results from X-ray emission measurements 
and Sunyaev-Zeldovich effect (inverse Compton scattering of CMB photons from free electrons in intra-cluster gas), the Bullet Cluster or even gravitational lensing [18].

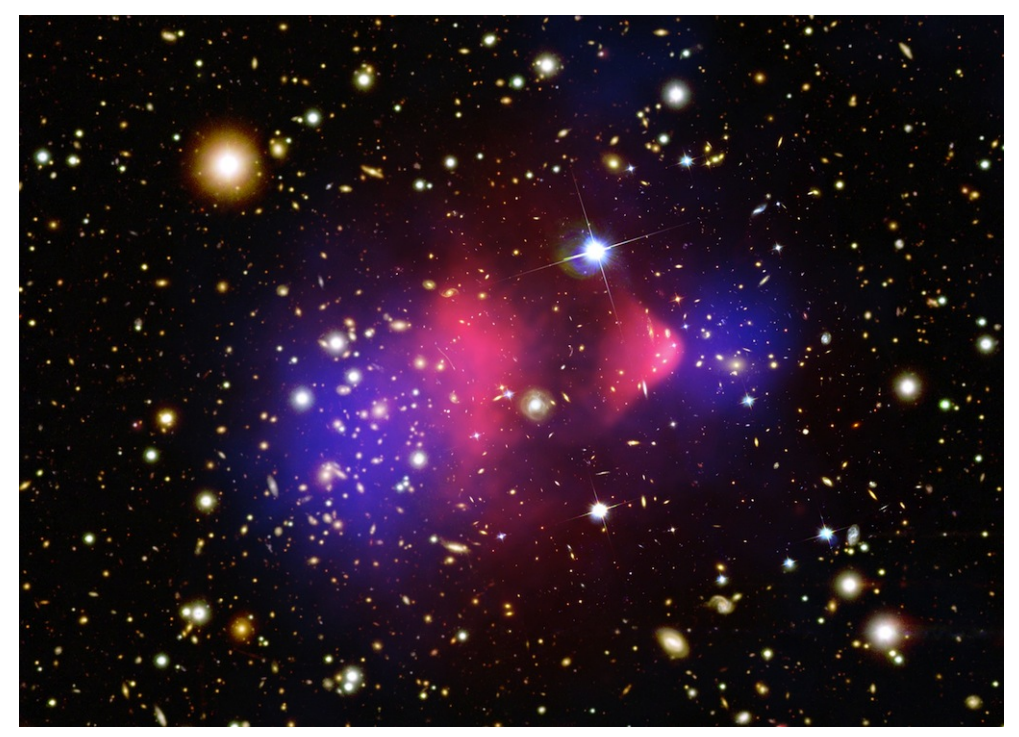

Figure 1.2: Composite image of the galaxy cluster 1E 0657-56 (aka "Bullet Cluster"). Optical image from Hubble space telescope and Magellan show galaxies in orange and white. X-ray emissions from hot intergalactic gas measured by the Chandra X-ray telescope is shown in false colour pink. Mass density inferred from weak gravitational lensing analysis is shown in false colour blue. Figure from [19].

\subsubsection{MACHOs}

MACHOS (Massive Compact Halo Objects) which may include black holes, stellar remnants and planets with insignificant contribution to the luminosity were proposed to explain the galaxy rotation curves. Searches for MACHOs are carried out using gravitational micro-lensing observations which look for a temporary increase in the 
observed intensity of background stars when MACHOs passing focus a greater amount of light into the telescope aperture. Such observations have set an upper limit of 25 $\%$ of the mass of dark halo that could be made up of MACHOs in the mass range of $2 \times 10^{-7}$ solar masses to 1 [20] and $40 \%$ on MACHOS of ten solar mass [21]. Limits on very massive halo objects (greater than 30 solar masses) do not exist and it is possible that they are made up of very massive black holes [22]. However given that MACHOs only explain a limited number of dark matter results, they are not among the favoured candidates.

\subsubsection{Neutrinos}

Standard model neutrinos in the early universe are expected to be relativistic and if relativistic particles formed the dominant matter component of the universe in the galaxy formation epoch, they would wash out any small scale structure and galaxies would not have formed [23].

\subsubsection{Axions}

Axions were originally proposed to solve the $\mathrm{CP}$ violation problem in strong interactions 24] 25]. They can potentially be the dark matter we are looking for. They would have been athermally produced in the early universe in a phase transition. Axions could be detected through conversion to photons in a strong magnetic field [26]. The ADMX experiment is looking for dark matter axions but has not found a signal so far [27]. 


\subsubsection{WIMPs}

An interesting and favoured candidate for cold dark matter is the WIMP (Weakly Interacting Massive Particle). WIMPs are a class of hypothetical particles which have an expected mass between a few $\mathrm{GeV}$ and a few $\mathrm{TeV}$, have an interaction cross section on the order of the weak scale, and are stable on cosmological time scales [28]. WIMPs could have been produced in the early universe along with other standard model particles but are massive enough to be frozen-out early (drop out of thermal equilibrium) and cooled down to non-relativistic speeds to aid structure formation. As a coincidence the required density to fit the $\Lambda$-CDM model arises when the interaction cross sections of WIMPs are of the weak scale 29].

Supersymmetry (SUSY), a new kind of symmetry which arises from unification of fundamental forces (electroweak and strong) gives WIMP like particles as a natural consequence. The lightest supersymmetric particle (LSP) is stable as a result of conservation of a new quantum number in SUSY which disallows conversion to standard model particles (except through annihilation with its antiparticle) [30] [31]. The neutralino is a highly favoured LSP WIMP candidate which occurs in a broad range of SUSY models and can produce the required dark matter density in the early universe. The parametric space for SUSY models is constrained from both experimental cosmology and accelerator particle physics 29. However, there are still regions of parameter space that are unexplored and it is possible that a dark matter particle could be lurking there. Various direct and indirect search experiments look for WIMPs; these are discussed in greater detail in section 1.3 . 


\subsubsection{Other exotic particles}

A number of other interesting candidates for dark matter have been proposed. Here we list a few for example. Under SUSY, the supersymmetric partner of the axion, 'axino' does not have a mass constraint and can be the LSP [32]. WIMPZILLA is another candidate; these are very massive particles that could be produced through different mechanisms at the end of the inflation period of the universe 33]. WIMPZILLAs have been proposed as solution to the ultra high energy cosmic ray problem. Although a number of other interesting and unique solutions to the dark matter problem have been proposed, they do not form the "natural", and hence favoured solutions at this point in time.

\subsection{Search for particle dark matter}

WIMPs are currently the most favoured candidate dark matter particle. Three main streams of experimental efforts are geared towards detecting WIMPs; they are 1) production of dark matter in high energy particle colliders, 2) indirect detection: looking for WIMP annihilation products 3) direct searches: looking for WIMP interaction in terrestrial detectors. The following subsections briefly review these three techniques.

\subsubsection{Production at high energy particle colliders}

Particle colliders attempt to create dark matter by colliding high energy particles (protons and their antiparticles). The total energy of the colliding particles in the centre of mass frame must be sufficiently high to access the dark matter particles' rest mass. This can happen in inelastic collisions where a fraction of the kinetic 
energy before the collision goes into making up the rest mass of a heavier dark matter particle. However, since dark matter interacts very rarely, collider experiments adopt a technique to detect "missing energy" rather than attempting to detect dark matter directly [34]. SUSY searches in particular have yielded strong constraints on the parameter space for WIMPs in preferred models, but no signal for dark matter has yet been observed. Although, discovery of a dark matter particle at a collider such as the LHC, would be highly significant for physics beyond the standard model, it need not make up all dark matter in the universe. Hence the three approaches discussed in this section are complementary to each other.

\subsubsection{Indirect searches}

If WIMPs are Majorana particles they would self annihilate and a signal could be observed in high WIMP density regions. If WIMPs are Dirac particles we could still observe their annihilation if their antiparticles are also abundant. Regions of high WIMP density which are close (greater solid angle) are the best places to look for annihilation products. Annihilation products could be SM fermions (electrons, protons, neutrinos and their antiparticles) or they could be photons. Some prominent indirect searches are mentioned below.

Looking for antimatter particles in cosmic radiation has been proposed as a technique for indirect detection due to minimal background and the universe's apparent matter excess over antimatter. PAMELA, a satellite borne experiment, and ATIC, a balloon borne experiment, have reported excesses in positron flux around $100 \mathrm{GeV}[35$ and electron/positron flux around a few hundred GeV [36] respectively, but other experiments (ground based HESS and LAT aboard FERMI satellite) have not observed 
a corresponding signal [37] [38].

Ice-cube and Super-K look for neutrinos from WIMP-annihilation in the centre of the sun, but no signal has yet been observed and only upper limits are set on such interactions [39] [40]. Ground based gamma ray telescopes like VERITAS also look for dark matter annihilation signals [41]. No incontrovertible evidence is observed as of yet. It must be noted however that signals observed in indirect searches cannot establish beyond doubt that the signal is from dark matter annihilation and not from yet unknown astrophysical phenomena.

\subsubsection{Direct searches}

Direct detection experiments look for WIMP interactions with terrestrial detectors. If WIMPs were produced in thermal equilibrium in the early universe then we expect them to interact inside our terrestrial detectors. WIMPs in our galaxy must be slow enough to be bound to the galaxy. From kinematics, the energy imparted by a WIMP to an electron is much smaller than that imparted to the nucleus, and hence direct search experiments look for nuclear recoils from WIMPs [42]. The WIMP direct detection rate depends on the local dark matter halo density, velocity distribution of WIMPs in the halo, WIMP mass, cross section of target nuclei with WIMPs and the target nuclei form factor [43]. The interaction cross section term in SUSY models is not constrained very well by theory and spans many orders of magnitude. Even in preferred models like the MSSM (Minimal Supersymmetric Standard Model), the interaction cross section spans many orders of magnitude [44]. Also, the cross sec-

tions predicted are very small (about $10^{-42}-10^{47} \mathrm{~cm}^{2}$ ) [44. The cross section of a WIMP with the nucleus can have a spin-independent and spin-dependent component. 
The spin-independent component is coherent across all nucleons for low energy transfers typically expected from WIMP interactions and scales as $A^{2}$ ( $A$ being the mass number), whereas the spin-dependent component results in cancellation of scattering amplitudes from nucleons of opposite spin and thus depends largely on the net spin of the nucleus. In order to compare results from different direct search experiments, the interaction cross section is normalized to a single nucleon and expressed in terms of spin-independent and spin-dependent components [29]. The search sensitivity for an experiment without background scales as $M \times T$ where $M$ is the target mass and $T$ is the exposure time, whereas for an experiment which requires subtraction of an estimated background, it scales as $\sqrt{M \times T}$ [29].

With the typical range of cross sections predicted by preferred SUSY models being very small, experiments need to have large target masses and need to run for long periods of time. The predicted event rates are considerably smaller than the background from environmental radioactivity and cosmic radiation. Hence, experiments go deep underground to reduce cosmogenic background (cosmic rays; especially cosmic ray muons) and shield the experiment well against environmental radioactivity. They also use highly radio-pure materials in the construction of the experiment and the target material itself. Despite this, the residual background can still dominate the expected signal rate; hence, background discrimination techniques become very important. The detection techniques used either measure ionization, lattice vibrations (phonons) or scintillation light or a combination of two of these.

Nuclear recoils and electron recoils produce different ionization and scintillation responses in detectors for a given total energy deposition. A term called the 'Quenching Factor' (QF) is used to describe the difference in the responses which is just a 
ratio of the signal magnitudes. This so called 'quenching' can be used to distinguish the two types of interactions. However, discrimination does not require this combination of signals. Other techniques including scintillation light pulse shape in noble liquids or the threshold effect generated by the different ionization density of particle tracks for nuclei compared to electrons can be used for discrimination. A very brief description of a select few direct dark matter search experiments of recent times are given in the following subsections while the results from recent searches are discussed in the last subsection of this chapter.

\section{DAMA/LIBRA}

The DAMA/LIBRA experiment uses $250 \mathrm{~kg}$ of NaI crystals at room temperature to measure scintillation light [45]. Since it is difficult to reject background events from electron recoil in this material on an event by event basis, they instead try to measure an annual modulation of count rate created by the annual modulation of the earth's relative velocity with respect to galactic coordinates due to its revolution around the sun. The DAMA/LIBRA collaboration published a positive signal for annual modulation for 2-6 keV from data over thirteen annual cycles [45] which some interpret as evidence for dark matter. However, multiple other experiments such as CDMS, EDELWEISS and XENON have ruled out this parameter space under standard assumptions regarding the astrophysical and particle properties of WIMPs. 


\section{SuperCDMS and EDELWEISS}

SuperCDMS and EDELWEISS operate semiconductor crystals (Ge) at cryogenic temperatures ( 20-50 $\mathrm{mK})$ and measure ionization and lattice vibrations. The key discrimination against background in SuperCDMS and EDELWEISS comes from the ratio of ionization over phonon signal. The CDMS experiment is discussed in greater detail in chapter 2. The low threshold and good energy resolution provide the basis for an excellent understanding of the residual background. This together with the efficient event-by-event discrimination is the origin for the great success of this technology [46] 47].

\section{XENON, LUX and DEAP}

Noble liquid detectors which are made of inert elements such as Xenon and Argon also provide unique advantages suited to dark matter detection. Apart from being inert, these materials are usually good scintillators and easily purifyable and the experiments are relatively easily scalable in size. The DEAP-3600 experiment is under construction at SNOLAB and will use a single phase liquid argon detector with a total mass of 3.6 tonnes (1 tonne fiducial) [48]. Discrimination against electron recoil background is achieved based on pulse shape. The LUX and XENON experiments use two phase xenon detectors with masses between 50 and $100 \mathrm{~kg}$ (fiducial) which provide good background discrimination 49,50$]$.

\section{PICASSO and COUPP}

The PICASSO experiment uses tiny droplets of $\mathrm{C}_{4} \mathrm{~F}_{10}$ embedded in a gel matrix.

Super heated liquid bubbles of $\mathrm{C}_{4} \mathrm{~F}_{10}$ are transformed into vapour phase by a small 
perturbation from a WIMP interaction depositing energy in the bubble [51]. This transformation generates a pressure wave which is detected by peizo-sensors. The COUPP project uses monolithic bubble chambers of superheated liquid $\left(\mathrm{CF}_{3} \mathrm{I}\right)$ rather than immersed droplets and also measures acoustic emission from interactions. Videocameras record the event allowing accurate 3D position reconstruction and re-compression [52].

\section{Results from direct searches}

Direct search results are expressed in terms of exclusion limits for no observed signal and as contours for an observed signal on a plot of WIMP-nucleon cross section versus WIMP-mass. Figure 1.3 shows such a plot for spin-independent WIMP-nucleon interaction for some prominent and recent direct dark matter search results. Two primary regions of interest are: WIMP masses $>50 \mathrm{GeV} / c^{2}$ and low mass WIMPs around $7 \mathrm{GeV} / c^{2}$ [53]; the former since it is favoured by WIMP models while the latter due to possible indications of a signal by multiple experiments (DAMA, CoGeNT, CRESST and CDMS II silicon) as well as predictions from some non-standard WIMP models such as asymmetric dark matter (with an asymmetry between dark matter particles and their antiparticles) [53]. 


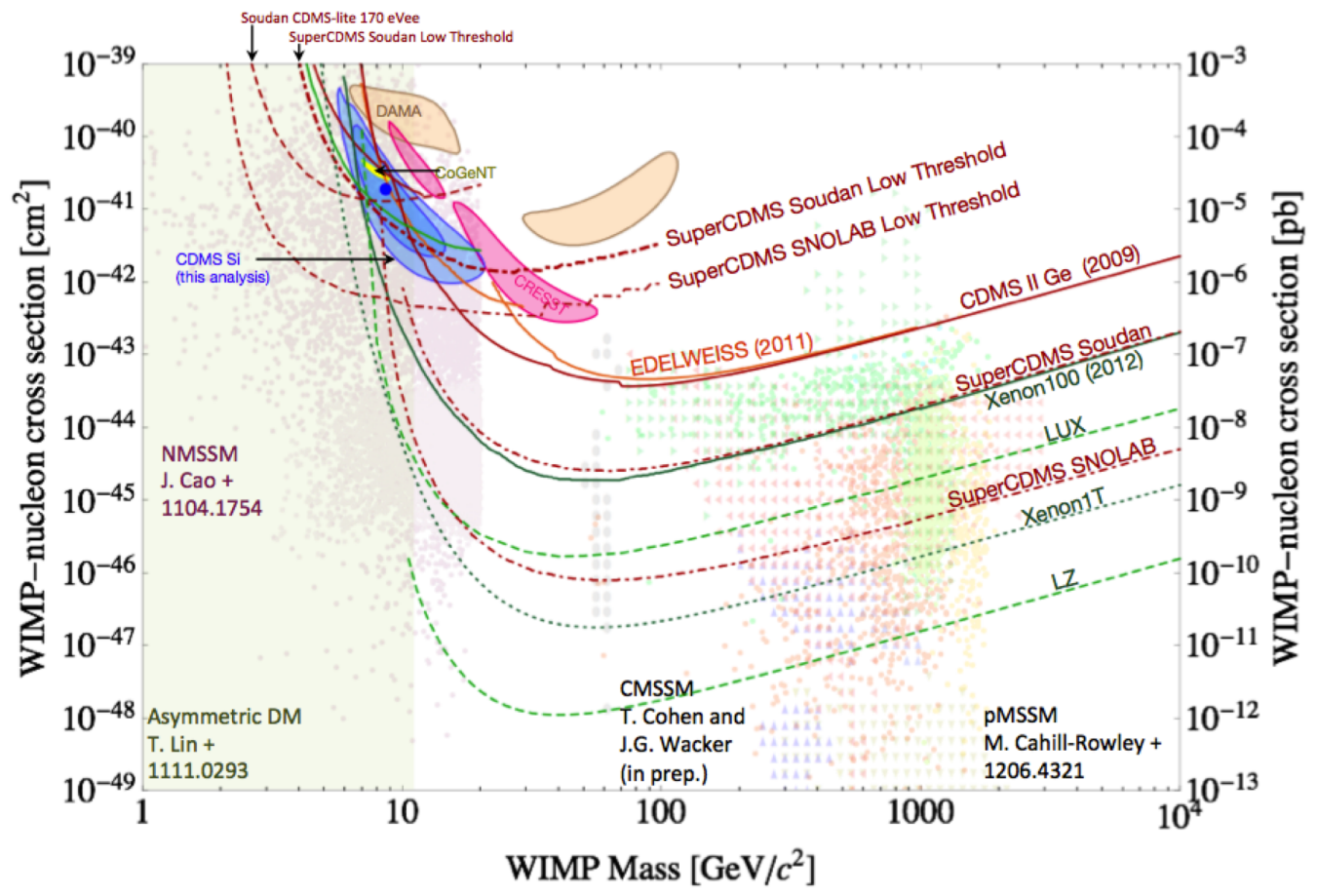

Figure 1.3: Recent results and projected limits for spin-independent WIMP-nucleon interactions from direct dark matter searches. Upper limits of $90 \%$ confidence level are plotted for CDMS II Soudan (red solid) [46] [54], EDELWEISS II (orange solid) [4] and XENON-100 (green solid) [55]. Contours with pink filling for CRESST II [56] and blue for CDMS II silicon data (both $68 \%$ and $90 \%$ CL) [57] are shown. Dotted regions show different SUSY models with LHC and Higgs constraints. Projected limits are shown in (dot-dashed red) for the CDMS-lite low threshold experiment, SuperCDMS Soudan and SuperCDMS SNOLAB. Projected limits are also shown for Xenon 1T (light green dashed), Lux (dark green dashed) and LZ (dark green dashed), the proposed successor of LUX . Figure from [53]. 
The current best limit for high mass WIMPs is set by XENON-100 and for low mass WIMPs below $6 \mathrm{GeV} / \mathrm{c}^{2}$, recent results by CDMSlite (CDMS Low Ionization Threshold Experiment), a SuperCDMS project which takes advantage of high LukeNeganov gain in the phonon energy while operating the detector at high voltage biases and obtains a lowered measurement threshold [58]. From the projected limits, we can expect that the low mass region where signals have been observed by multiple experiments will be mostly probed by SuperCDMS Soudan low threshold analysis and will eventually be confirmed or completely ruled out by the SuperCDMS SNOLAB low threshold experiment. With a host of new experiments with complementary technologies, direct dark matter searches in the near future are expected to probe most of the parameter space predicted by preferred dark matter models. 


\section{Chapter 2}

\section{The CDMS Experiment}

\section{$2.1 \quad$ Introduction}

The CDMS (Cryogenic Dark Matter Search) experiment has been one of the leading direct dark matter search experiments for over a decade [54,59 61]. The CDMS experiment in its initial phase (CDMS- I) was operated at the Stanford Underground Facility (SUF) and the final results from this phase published in 2002 set world leading limits for spin-independent WIMP-nucleon elastic scattering cross section for WIMP with masses between $10-70 \mathrm{GeV} / c^{2}$ [59]. The SUF is a tunnel 10.6 $\mathrm{m}$ below ground beneath the Stanford university campus. The $17 \mathrm{~m}$ w.e (meter water equivalent) of earth above the experiment completely eliminates the primary hadron component of cosmic radiation while reducing the muon component by a factor of $\sim 5$ [62. This phase of the experiment used four BLIP (Berkley Large Ionization and Phonon mediated) germanium detectors and one ZIP (Z-sensitive Ionization and Phonon mediated) silicon detector. The BLIP detectors are high 
purity germanium crystals instrumented with charge sensing electrodes and neutrontransmutation-doped (NTD) germanium thermistors to measure thermal phonons. The ZIP detector consist of a cylindrical Si crystal made with two ionization sensors on one side and four athermal phonon sensors made of tungsten transition-edgesensors (TES) with phonon aluminium phonon collectors [59].

The next phase (CDMS II) began at SUF as well but was moved to an underground lab at the Soudan mine in northern Minnesota since the experiment's sensitivity was limited by cosmogenic background rate. The lab at the Soudan mine is at a depth of $780 \mathrm{~m}$ (2090 m w.e) and reduces the cosmic ray muon flux by a factor of $\sim 50,000$ compared to the surface [63]. The CDMS II experiment at Soudan began with a single stack of six detectors (called a 'tower') in 2002 and the active target was then increased to two towers and finally five towers, setting the best exclusion limits with the data gathered through these phases [63] [13]. CDMS II at Soudan operated a combination of 19 germanium and 11 silicon ZIP detectors between 2006 and 200861 and produced the strongest limits on spin-independent WIMP nucleon cross section only rivalled by XENON-10 at low energies [64]. The experiment was eventually limited by surface event background and thus improved detectors were needed.

SuperCDMS at Soudan is the successor of CDMS II and currently runs with a total target mass of $\sim 9 \mathrm{~kg}$ of a new generation of iZIP (interleaved Z-sensitive Ionization and Phonon mediated) germanium detectors. The iZIP detectors are cylindrical Ge crystals that have two charge sensors and four phonon sensors on each side. They are discussed in greater detail in section 2.2. The sensitivity of SuperCDMS at Soudan is expected to become background limited from cosmogenic neutrons within a few years of running [65. In its next phase SuperCDMS plans to move to SNOLAB (Sudbury, 
Ontario) to cut down the cosmic ray muon flux. The SNOLAB experimental site located about $2 \mathrm{~km}$ underground ( $6000 \mathrm{~m}$ w.e overburden) is expected to suppress the muon-induced neutron background by two orders of magnitude [66]. It also plans to run $\sim 100-200 \mathrm{~kg}$ of new, more massive iZIP detectors which will allow it to scan spin-independent WIMP-nucleon elastic scattering cross sections of $10^{-44}-10^{-46} \mathrm{~cm}^{2}$ 66.

\subsection{CDMS detectors}

CDMS detectors have evolved over its two decades of dark matter search. However, the key feature of CDMS detector technology has always been the simultaneous measurement of ionization and phonon signals from particle interactions inside semiconductor crystals. This allows the determination of true recoil energy as well discrimination between electron recoils (ER) and nuclear recoils (NR). The nuclear recoils produce a smaller ionization signal compared to electron recoils for interactions of same recoil energy. Figure 2.1 shows the plot of ionization signal versus recoil energy for electron recoil events from gammas and nuclear recoils from neutron interactions. It is common to plot the yield (ionization energy / recoil energy) versus recoil energy as shown in figure 2.2. The yield of electron recoils is normalized to one. The yield for nuclear recoils is lower due to smaller ionization for the same true recoil energy and ends up $\sim 0.3$. The nuclear recoils from WIMPs are expected to lie in the same region as neutrons. 


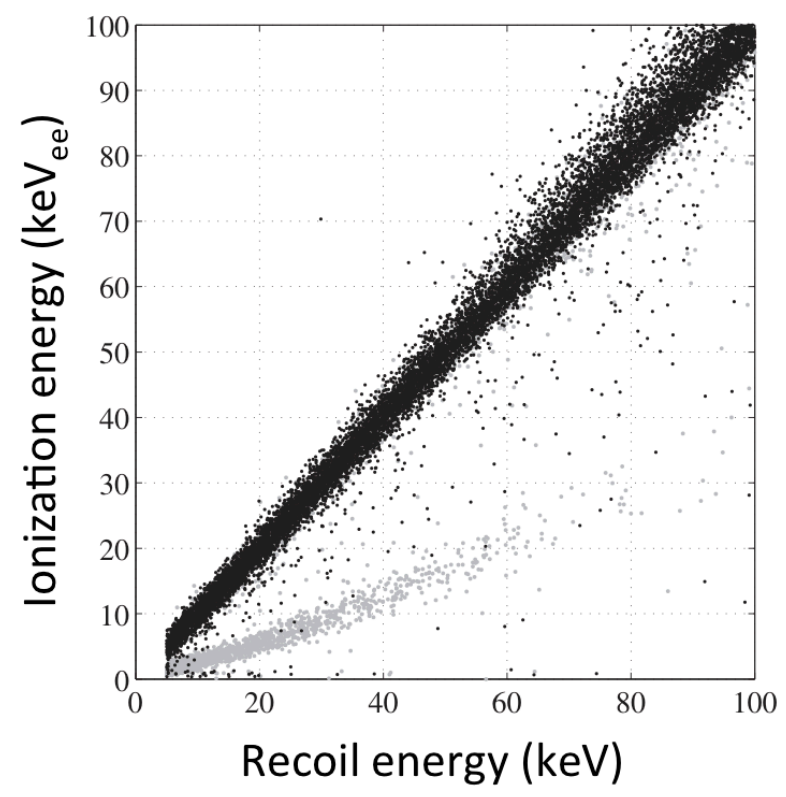

Figure 2.1: Ionization versus recoil energy in an ZIP detector. The black events are from a dataset with a ${ }^{133} \mathrm{Ba}$ source which emits gammas only and the grey events are from a dataset with a ${ }^{252} C f$ source which emits neutrons and gammas. Figure from $[60]$.

As described earlier, the first phase of CDMS used BLIP detectors with NTD sensors which measured thermal phonons. Thermal phonons provide a good energy estimation but tell us very little about the position of the event in the detector. The ZIP detector uses tungsten transition edge sensors for phonon measurement. Schematics of the two faces of the cylindrical ZIP detector are shown in figure 2.3 . 


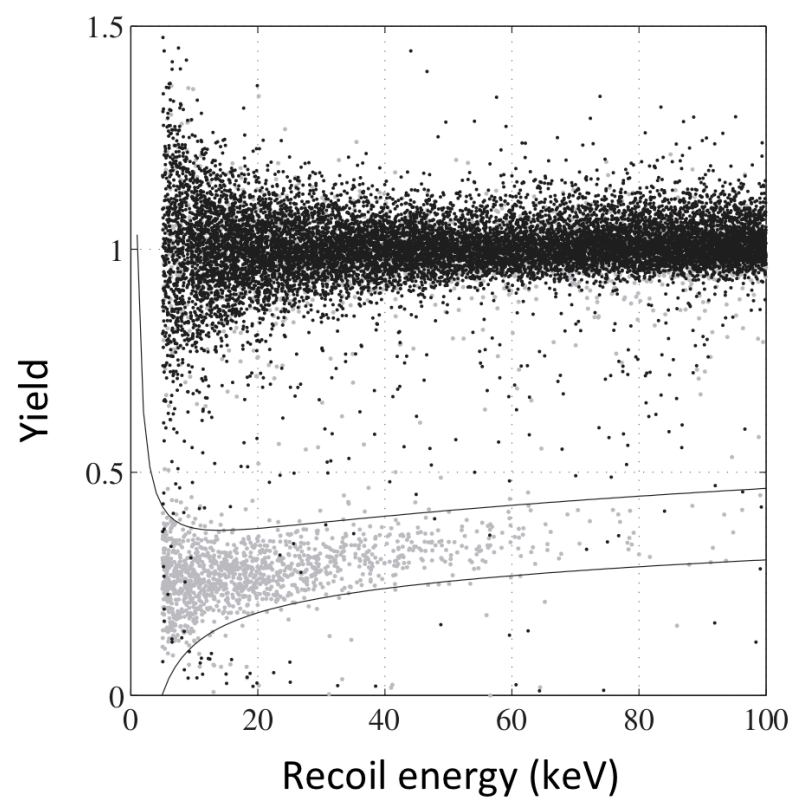

Figure 2.2: Yield versus recoil energy in an ZIP detector. The black and grey events are the same data selection as in figure 2.1. The lines represent the $2 \sigma$ bounds for the NR band. Figure from [60].

The charge face has two charge sensors $Q_{\text {inner }}$ and $Q_{\text {outer }}$, while the phonon face has four phonon sensors (labelled A,B,C and D) made up of TESs. The TES resistance changes drastically with temperature when in the transition region (around 50 -100 $\mathrm{mK})$, and hence it can act as a very sensitive temperature sensor. These sensors are photolithographically patterned on the crystal surface. The charge sensors are electrodes made of aluminium. The $Q_{\text {outer }}$ electrode acts as a guard ring allowing us to veto events happening close to the cylindrical wall. Bias voltage is applied to the charge electrodes to generate a field of a few volts/cm to drift the charges across the crystal [60]. 

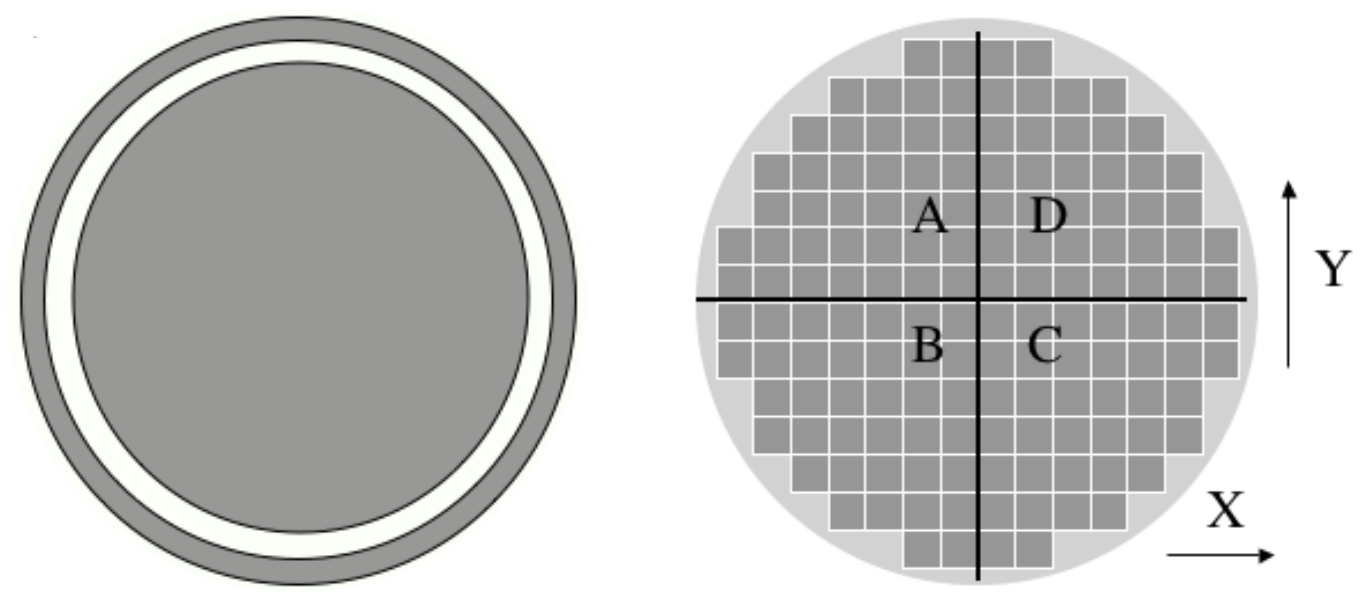

Figure 2.3: The charge face (left) and the phonon face (right) of the CDMS ZIP detector. The inner disc is the main charge sensor while the outer ring serves as a guard. The arrangement of phonon sensors into four quadrants allows X-Y position reconstruction for each event. Figures from [60].

ZIP detectors allow us to measure athermal phonons which carry information about the position of an event. ZIP detectors are also sensitive to the differences in measured phonon pulse shapes for different kinds of interactions. An interaction in the detector produces high frequency phonons which undergo quasi-diffusive propagation [60]. The net effect of the processes involved is to create a ball of phonons expanding from the point of interaction at $1 / 3^{r d}$ the speed of sound 60 . Once the phonons decay to frequencies lower than about $1 \mathrm{THz}$, their mean free path becomes comparable to the dimensions of the detector and they are then referred to as ballistic phonons [60]. The ballistic phonons propagate at the speed of sound and reflect off the crystal surfaces unless they are absorbed by the aluminium fins which are attached to the tungsten TESs to increase phonon collection. The ballistic phonons eventually thermalize in the detector. 
Electron-hole pairs created from the interaction drift due to the electric field inside the crystal and give rise to additional ballistic phonons called Neganov-TrofimovLuke phonons (also referred to as 'Luke phonons') [60]. The energy content of Luke phonons is proportional to the number of charge carriers drifting and the potential difference between electrodes $[60]$. Since electron recoils create a greater number of electron-hole pairs for an interaction (also referred to as an 'event') of a given true recoil energy compared to nuclear recoils, they also produce a greater number of Luke phonons which are ballistic [67]. Since the charges from ionization drift almost instantaneously after the interaction, the Luke phonons produced due to their drift constitute the early (right after the interaction) population of ballistic phonons. The additional energy in Luke phonons thus leads to a faster leading edge of the phonon pulse for electron recoils [60].

For events happening close to the surface which has metal electrodes, either on the top or bottom surfaces of the detector, the high frequency phonons down-convert quickly to ballistic phonons due to interaction with free electrons in metal. This again leads to a faster phonon leading edge compared to events happening in the bulk of the detector [60]. Events happening close to the surface are also prone to incomplete charge collection and in turn tend to have a reduced yield. The yield can be low enough to match that of a NR, generating background in the signal region. The ability to discriminate against surface electron recoils using differences in pulse shape provides a good handle on this background. However, despite that, CDMS II was limited by the residual surface event background.

In order to further reduce the surface event background and to increase the overall detection efficiency a new detector design called the iZIP was adopted for the next 
phase of the experiment. The iZIP detector features ionization electrodes interleaved with phonon sensors on both sides of the detector (see figure 2.4). The phonon sensors also act as the ground electrode for the ionization measurement. The iZIP design currently employed is the result of several revisions from its initial design pictured in [68].
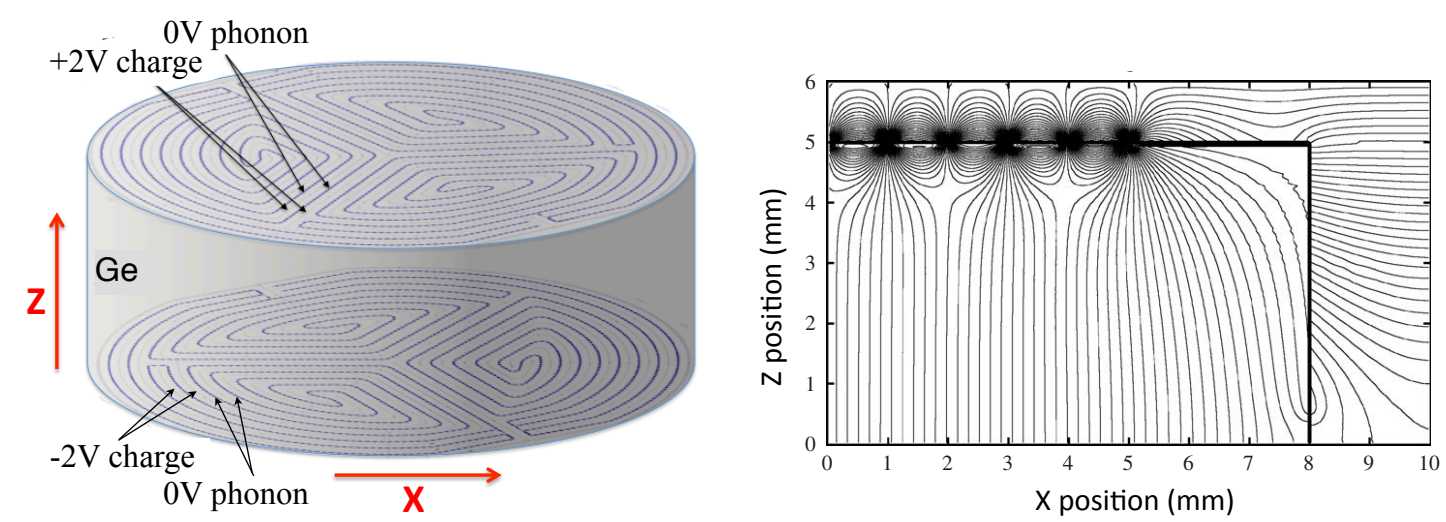

Figure 2.4: The iZIP detector (left) 69]. Interleaved charge and phonon sensors can be seen on either side. The mercedes like layout of the phonon sensors is comprised of three inner sensors forming three equal sections of a circle and one phonon sensor as an outer ring. The figure on the right shows the electric field profile in the bulk and close to the top surface (Figure from 68$]$ ).

This design creates a bulk field between the two opposite polarity charge electrodes on the top and bottom surfaces while also creating a surface field between the ionization and ground electrodes (phonon sensors) on both sides. The surface field extends $\sim 1 \mathrm{~mm}$ or so into the crystal. In this design an event happening in the bulk of the detector produces a signal in the charge sensors on both sides of the detector while an event happening close to either the top or bottom surface produces a signal 
only on charge sensors on the respective side, thus allowing surface event discrimination. The phonon sensors are tungsten TESs with aluminium fins just as in the case of the ZIP, albeit with a modified layout. The design of the four phonon sensors on both sides is the same, however they are rotated by $60^{\circ}$ with respect to each other to improve the position reconstruction and to reduce the redundancy of position information in phonon signals. The iZIP detectors have shown very good surface event discrimination ability and have become the design of choice for SuperCDMS at SNOLAB [69].

\subsubsection{Ionization signal}

An interaction happening in the detector creates electron hole pairs. A part of the energy from the interaction is spent on driving charge carriers across the band gap while the rest is dissipated as phonons [63]. The charge electrodes are aluminium rails on the crystal faces separated by a thin layer of amorphous silicon to improve charge collection of surface events by reducing carrier back-diffusion into the adjacent

electrode [63]. The charge carriers drift under the influence of the electric field to the electrode and are collected. The charge amplifier used to readout the ionization signal is discussed in chapter 5. The fall time of charge pulses is determined by the time constant of the feedback circuit and is $\sim 40 \mu \mathrm{s}$ [70].

The reconstruction of charge pulses to extract energy information is carried out using an optimal filter algorithm. The optimal filter algorithm makes use of the fact that the pulse shape of the charge signal remains constant since the time constants in the recorded pulse are dictated by the electronics while the noise is random and predominantly Gaussian [63]. The reconstruction is achieved by fitting a fixed template 
to the observed pulse in the frequency domain by accounting for variations of noise and signal power $[63]$. The template is created by averaging carefully selected good pulses. In its current form the optimal filtering algorithm also performs crosstalk correction by accounting for the capacitive crosstalk between $Q_{\text {inner }}$ and $Q_{\text {outer }}$ channels. In order to do this it uses two pulse templates to make a fit to the observed pulse on each channel; one for a signal observed on the given channel and one for the crosstalk 63.

Physical parameters for each event after the reconstruction are called 'reduced quantities' (RQs) and are stored in ROOT files. The RQ's are also made available on MATLAB for data analysis using a package of scripts written by the CDMS collaboration called CAP (CDMS Analysis Package). CAP also has scripts for routinely used functions in CDMS data analysis and is written in a way to optimize memory requirements and computational speed.

\subsubsection{Phonon signal}

The phonon energy collected by the sensors originates from primary recoil phonons, carrier relaxation phonons and Luke phonons. Primary recoil phonons make up all the energy except that which is required to drive the electron-hole pairs across the band gap. They are comprised of high energy optical phonons as well as lower energy acoustic phonons [63]. The carrier relaxation phonons are produced when the charge carriers recombine at the electrodes and the energy originally lost to electron hole pair production is regained. The work done by the electric field to drift the charge carriers across the crystal is fed back to the phonon system as phonon radiation 63 (or Luke phonons). As mentioned before, the energy content in Luke phonons depends 
on number of charge carriers that drift and the applied voltage. The total phonon energy $\left(E_{p}\right)$ measured is the sum of the recoil energy $\left(E_{R}\right)$ and the Luke phonon contribution (for a CDMS II ZIP) is given by:

$$
\mathrm{E}_{\mathrm{p}}=\mathrm{E}_{\mathrm{R}}+\mathrm{e} \mathrm{V}_{\text {bias }} \mathrm{N}_{\mathrm{q}}
$$

where $\mathrm{V}_{\text {bias }}$ is the voltage bias applied (across the bulk (in ZIP)) and $\mathrm{N}_{q}$ is the number of electron-hole pairs. $N_{q}$ can be expressed as the total ionization energy measured $\left(\mathrm{E}_{q}\right)$ divided by the energy required to create an electron-hole pair $(\epsilon)$. The total phonon energy is then given by:

$$
\mathrm{E}_{\mathrm{p}}=\mathrm{E}_{\mathrm{R}}+\frac{\mathrm{e} \mathrm{V}_{\text {bias }}}{\epsilon} \mathrm{E}_{\mathrm{q}}
$$

In the case of an iZIP detector an ideal bulk event has the same Luke phonon contribution, whereas a perfect surface event has half this contribution due to the voltage drop between the bias and ground electrode being half of that between two bias electrodes on opposite sides of the crystal. However, if an event is neither perfectly bulk nor surface then there are two contributions to the Luke phonons, namely the symmetric contribution (common to both sides of crystal) and an asymmetric contribution (only on one side). In fact, the Luke phonon contribution can be expressed in terms of the symmetric and asymmetric charge collection for an event happening anywhere in the detector. The Luke phonon component expressed as the sum of contributions form asymmetric and symmetric charge collection is given by:

$$
\mathrm{E}_{\mathrm{L}}=2 \mathrm{e} \mathrm{V}_{\text {bias }} \mathrm{N}_{\mathrm{q}, \text { sym }}+\mathrm{e} \mathrm{V}_{\text {bias }} \mathrm{N}_{\mathrm{q} \text {,asym }}
$$


where $N_{q, s y m}$ and $N_{q, a s y m}$ are the number of charge carriers in the symmetric and asymmetric components of the charge signal. This can be expressed in terms of total charge collection of side $1\left(\mathrm{E}_{q 1}\right)$ and side $2\left(\mathrm{E}_{q 2}\right)$ as:

$$
\mathrm{E}_{\mathrm{L}}=\frac{2 \mathrm{e} \mathrm{V}_{\mathrm{bias}}}{\epsilon} \min \left(\mathrm{E}_{\mathrm{q} 1}, \mathrm{E}_{\mathrm{q} 2}\right)+\frac{\mathrm{e} \mathrm{V}_{\mathrm{bias}}}{\epsilon}\left|\mathrm{E}_{\mathrm{q} 1}-\mathrm{E}_{\mathrm{q} 2}\right|
$$

Note that the voltage between the bias and ground electrodes is $\mathrm{V}_{\text {bias }}$, whereas the voltage between the bias electrodes on opposite sides of the crystal is $2 \times \mathrm{V}_{\text {bias }}$. Since twice the minimum signal plus the difference between the two signals is just the sum of two signals, the total phonon energy measured in an iZIP is thus given by:

$$
\mathrm{E}_{\mathrm{p}}=\mathrm{E}_{\mathrm{r}}+\frac{\mathrm{e} \mathrm{V}_{\mathrm{bias}}}{\epsilon}\left(\mathrm{E}_{\mathrm{q} 1}+\mathrm{E}_{\mathrm{q} 2}\right)
$$

The ZIP and iZIP class of detectors use transition edge sensors (TESs). The TES is a tungsten film that is maintained in its superconducting transition region where the resistance is very sensitive to a change in the temperature. If the TES is voltage biased, a change in the temperature caused by phonon absorption causes a change in the sensor current, which in turn can be amplified and read out. The phonon sensors consist of a superconducting tungsten TESs with attached aluminum fins to improve phonon collection in the sensor [63].

The phonon readout system is shown in figure 2.5. The shunt resistance $R_{s h}$ is about $20 \mathrm{~m} \Omega$ while the operating sensor resistance under transition is about 200 $\mathrm{m} \Omega$. The bias current $\mathrm{I}_{b}$ is set such that the TES is in its transition region. Since shunt resistance is small, most of the bias current, $I_{b}$, passes through the shunt resistor and in effect voltage biases the TES. When an interaction happens in the 
detector, the temperature of the TES rises. This changes the resistance of the TES, in turn changing the current through the TES and the input coil (see figure 2.5). The change in input coil current creates a change in the magnetic flux coupled to a Superconducting Quantum Interference Device (SQUID). The SQUID translates this flux change into a voltage change which gets amplified and fed to the feedback coil as the current $I_{f}$. The feedback coil provides negative feedback and has $1 / 10^{\text {th }}$ the number of turns as the input coil. This means the feedback coil current required to compensate the flux change through the SQUID is 10 times the input coil current, resulting in a transimpedance of $10 \times R_{f b}[63]$.

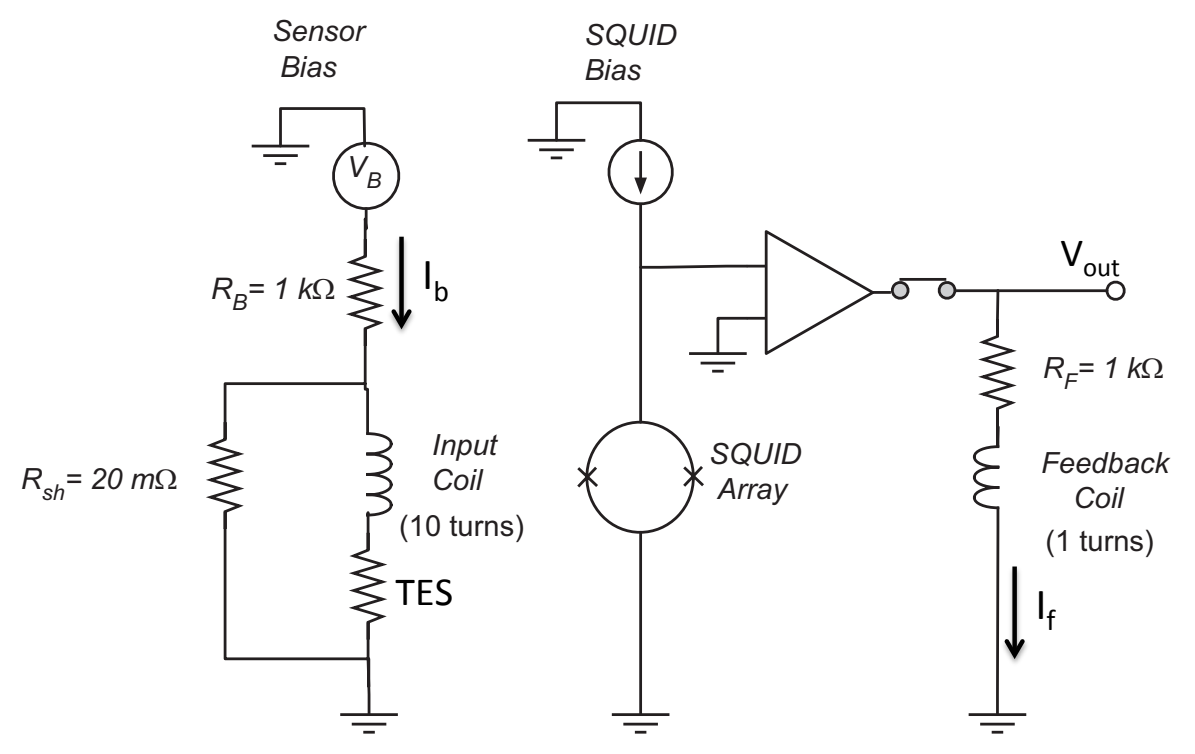

Figure 2.5: Phonon readout system using a TES sensor and SQUID based amplification system. Figure from [70].

Phonon energy reconstruction is also achieved using the optimal filter (OF) algorithm. However phonon pulse shapes vary depending on the nature of interaction 
and the position of the event in the detector. Due to this we observe nonlinearities in the reconstructed amplitudes and energies [63]. The nonlinearities in reconstructed energies are corrected for in the analysis, while the differences in pulse shape depending on the nature of interaction are used to discriminate against background events. Start times obtained from the optimal filter algorithm are also affected by systematics. Time domain fits provide a better alternative for accurate reconstruction of start times, although the energy reconstruction with their use suffers from low resolution [63]. SuperCDMS has developed a non stationary optimal filter (NSOF) algorithm which manages to reduce position dependence in the energy reconstruction considerably. Chapter 6 includes a more detailed discussion of the NSOF algorithm and its usefulness in the work carried out towards this thesis.

\subsection{Backgrounds}

Since WIMP events in terrestrial detectors are rare, direct dark detection experiments adopt various techniques to combat background. As mentioned earlier, the $2090 \mathrm{~m}$ w.e overburden at the Soudan underground lab already reduces the neutron flux induced by cosmic ray muons significantly. Natural sources of background include high energy neutrons from cosmic ray muon interactions in surrounding material and cavern rock, gamma rays and neutrons from ambient radioactivity, electrons, photons and alphas from surrounding material, recoiling ${ }^{206} \mathrm{~Pb}$ nuclei from radon contamination as well as radioactive contamination of the detector itself [60]. The CDMS II experiment employed a combination of active and passive shielding to further reduce background. 


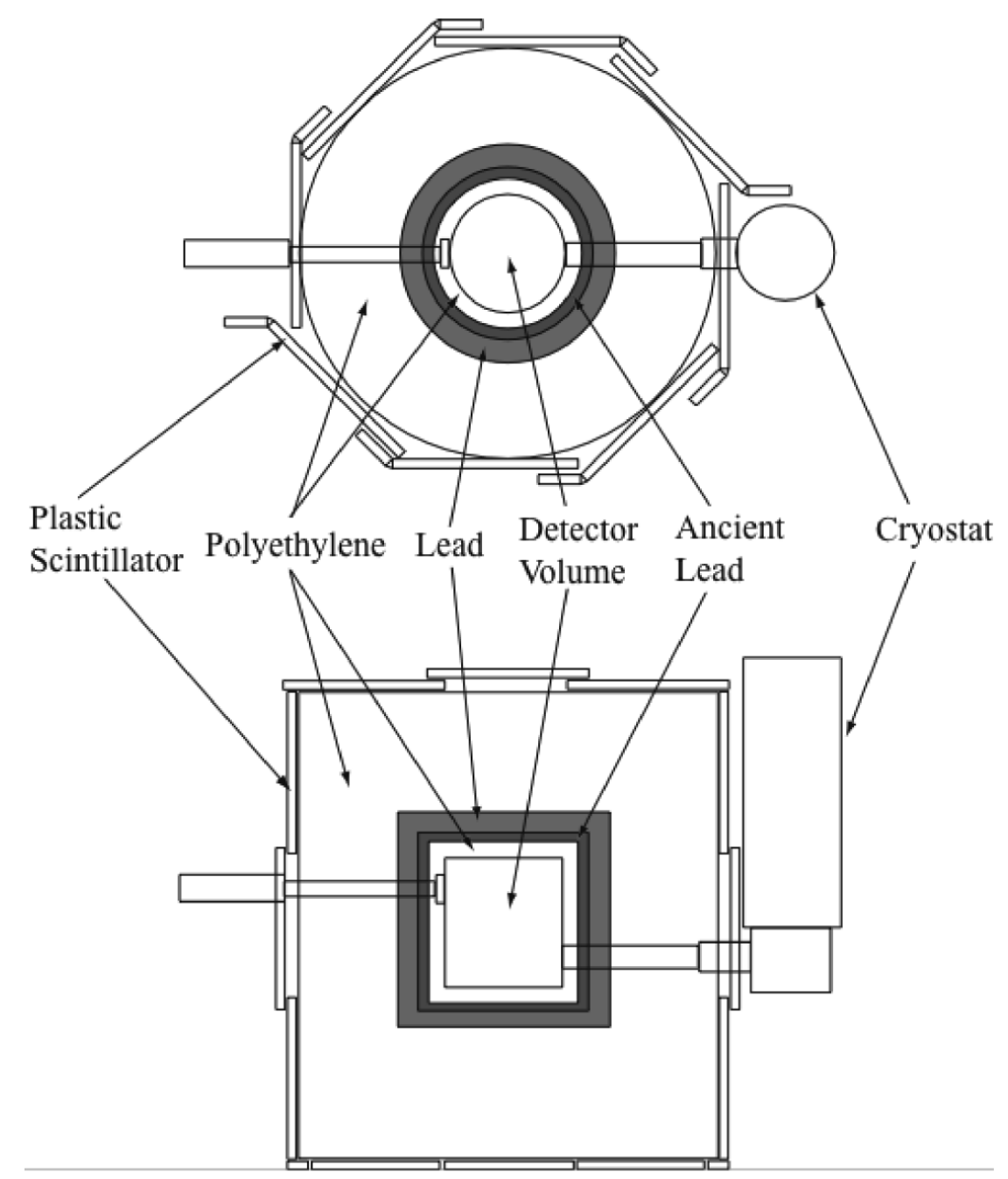

Figure 2.6: CDMS II active and passive shielding. Figure from [60].

Figure 2.6 shows the CDMS II shielding setup. Surrounding the detector volume are several layers of shielding. Outermost is the active muon veto which uses panels of plastic scintillator coupled to PMTs. The panels are arranged in a manner to maximize the efficiency of tagging cosmic ray muons. The active muon veto tags cosmic ray muons with nearly $100 \%$ efficiency [60]. Next is a $40 \mathrm{~cm}$ thick polyethylene 
cylinder which moderates neutrons from radioactive decay from cavern rock. Inside this there are two layers of lead shielding: a $22 \mathrm{~cm}$ layer of lead with $4.5 \mathrm{~cm}$ layer of ancient lead (low ${ }^{210} \mathrm{~Pb}$ content) to shield from gammas. Inside the lead shielding there is another $10 \mathrm{~cm}$ polyethylene cylinder to moderate neutrons from cascadeshowers due to high energy neutrons passing through the outer polyethylene shielding and interacting in the lead. Air surrounding the fridge is continuously purged with nitrogen to prevent radon accumulation.

To keep the radioactivity from the hardware inside the icebox to a minimum, all components are made radio-pure materials. Detectors and all the supporting hardware are kept in nitrogen purge cabinets when not in use to minimize radon contamination. All of the apparatus described, including the shielding, are placed in a Faraday cage ('RF room') which is meant to minimize electromagnetic interference. The RF room and the access to the RF room are maintained as clean rooms.

As discussed in section 2.2, surface events form a major source of background in our detectors which is mitigated by the improvements in detector design (the iZIP) as well as use of analysis techniques based on difference in phonon pulse shapes. The residual background from natural sources including contamination of detector surfaces is estimated by monte-carlo simulations and accounted for in the results [60]. Background from multiple scatter events close to the surface within a single detector may become significant for the SuperCDMS SNOLAB phase of the experiment. A modified detector design to discriminate against this background is introduced in chapter 5 and forms the bulk of the work carried out towards this thesis. 


\section{Chapter 3}

\section{Queen's Test Facility}

SuperCDMS detectors are tested and characterized as a part of the production chain before being deployed for dark matter detection. Facilities are established in various locations to execute these tests as well as to participate in research and development for the experiment. The following section describes briefly some of the main infrastructure at the SuperCDMS 'Queen's Test Facility' (QTF). A more detailed account of the Queen's test facility infrastructure can be found in Joseph Fox's masters thesis (see 71$])$.

\subsection{Infrastructure at QTF}

\subsubsection{Cryostat}

Since our detectors run at temperatures of a few milli-Kelvin $(\mathrm{mK})$ we have to use a cryostat to cool them down to these temperatures. QTF uses a dry dilution refrigerator with a pulse tube cooler to do this. Unlike a traditional dilution refrigerator, 
the 'dry' dilution refrigerator with pulse tube cooler does not use any liquid cryogens. The temperature usually achieved with LN or LHe is here achieved with the use of a pulse tube cooler. The dilution refrigerator uses special properties of a ${ }^{3} \mathrm{He}^{-}{ }^{4} \mathrm{He}$ mixture to cool down to temperatures below $10 \mathrm{mK}$. The phase diagram of the ${ }^{3} \mathrm{He}^{-}{ }^{4} \mathrm{He}$ mixture at low temperatures is shown in figure 3.1. At temperatures below $0.867 \mathrm{~K}$ the mixture undergoes phase separation [72]. The mixture then exists in two phases. A ${ }^{3} \mathrm{He}$ rich phase $\left(\sim 94 \%{ }^{3} \mathrm{He}\right)$ and a ${ }^{3} \mathrm{He}$ dilute phase $\left(\sim 6 \%{ }^{3} \mathrm{He}\right)$. Since ${ }^{4} \mathrm{He}$ is heavier than ${ }^{3} \mathrm{He}$, the two phases are physically separated by gravity. However the enthalpy of the ${ }^{3} \mathrm{He}$ in the dilute phase is much higher than its enthalpy in rich phase while ${ }^{4} \mathrm{He}$ at these temperatures $(<0.5 \mathrm{~K})$ does not contribute to the specific heat capacity of the mixture [73]. Diffusion of ${ }^{3} \mathrm{He}$ from its rich phase to dilute phase can be achieved by creating a osmotic pressure gradient. Due to the difference in enthalpies this is a endothermic process and this drives the cooling.

When the mixture is condensed and phase separated inside the cryostat, cooling can be achieved by circulating the mixture using a pump. Since ${ }^{3} \mathrm{He}$ easily evaporates, it is pumped out and circulated leaving most of the ${ }^{4} \mathrm{He}$ behind. The pumping continues until only ${ }^{3} \mathrm{He}$ is being circulated. This takes away the heat from within the cryostat and cools the samples/detectors. 


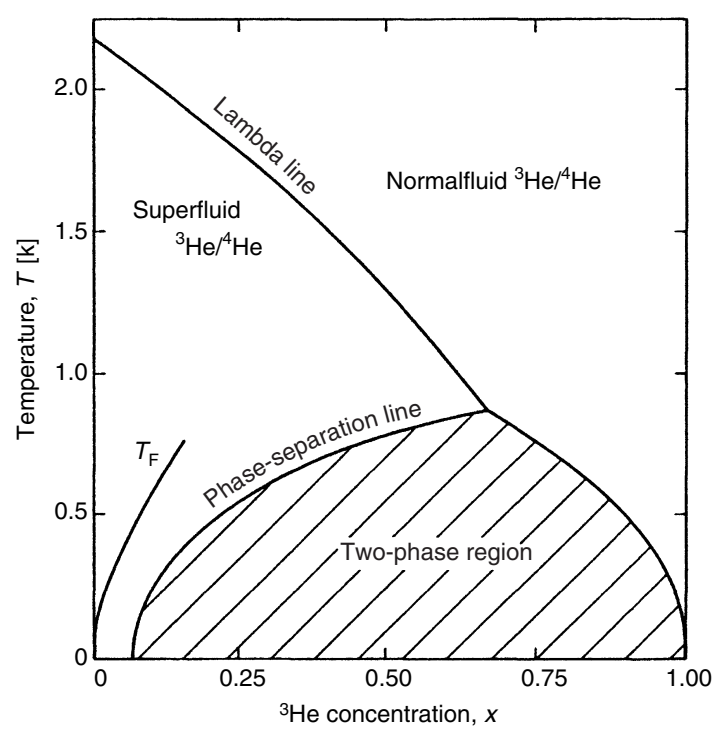

Figure 3.1: Phase diagram of ${ }^{3} \mathrm{He}^{-}{ }^{4} \mathrm{He}$ mixture at low temperatures for saturated vapour pressures. Figure from 72$]$.

A picture of the different temperature stages (plates) in our cryostat is shown in Figure 3.2 . The inside of the cryostat needs to be under vacuum to avoid a thermal link between the different temperature stages. For this an outer vacuum chamber (OVC) is used. The OVC is a big can with O-rings that vacuum-seals the inside of the cryostat from the surroundings. 


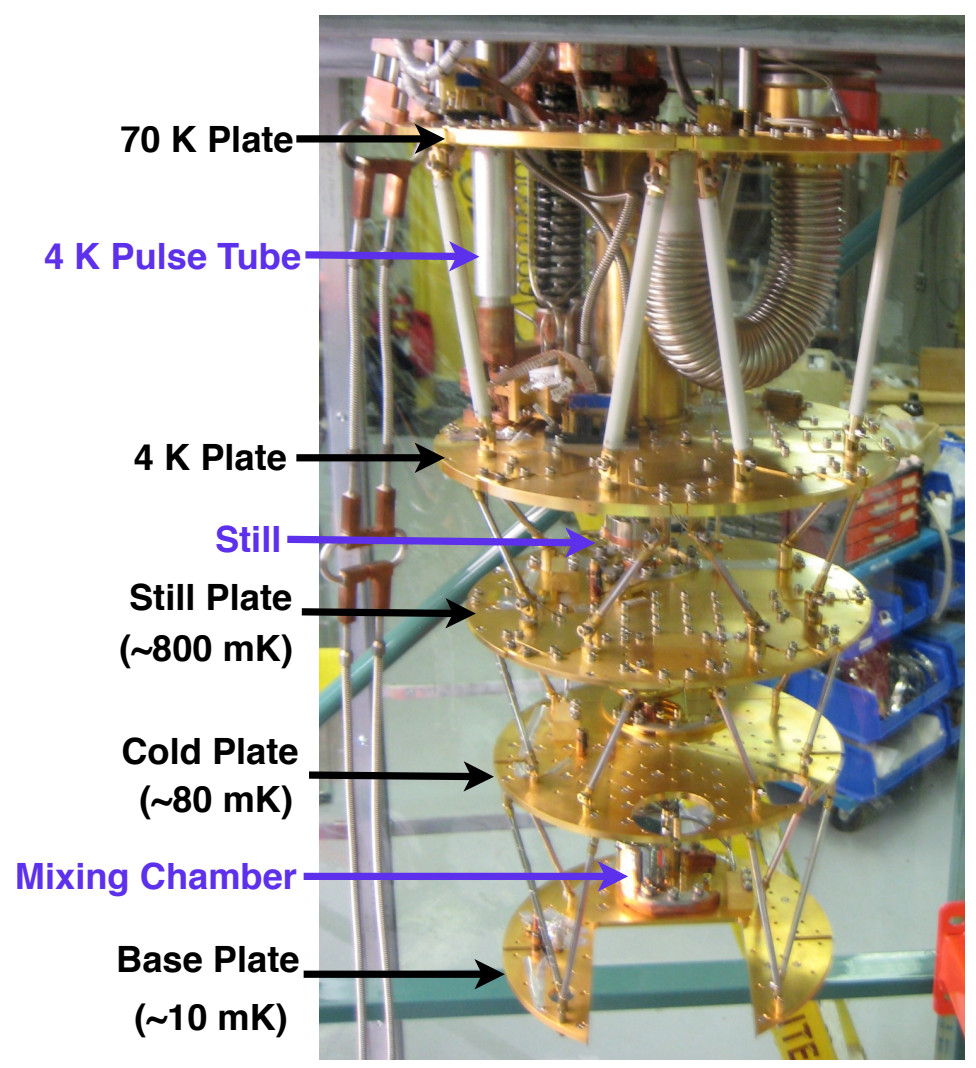

Figure 3.2: Different temperature stages inside of the cryostat. Note: the tower with detectors is not installed in this picture.

The $70 \mathrm{~K}, 4 \mathrm{~K}$ and $600 \mathrm{mK}$ temperature stages have radiation shields (reflective cans) which are attached to the respective plates in order to avoid radiation from higher temperature stages shining on the lower temperature stages and the detectors. The pulse tube cooler along with its helium compressor allows us to cool down and maintain the nominal temperatures on the $4 \mathrm{~K}$ and $70 \mathrm{~K}$ (usually around $60 \mathrm{~K}$ ) plates without the use of the mixture. The lower temperature stages are cooled in the 'precool' stage by sending in cold mixture to the base plate. Once we reach $\sim 10 \mathrm{~K}$ on the 
base plate, the condensing can be started. The mixture then follows a different route to the mixing chamber (container on the base plate; see figure 3.2) along which it is cooled down using heat exchangers and by passing through impedances followed by expansion into larger volumes. The mixture then condenses in the mixing chamber and in the still (container on the still plate). The mixture is then in a state where it can undergo phase separation once the optimum temperature is reached and the cooling is achieved as described before. We are able to reach temperatures of $\sim 10$ $\mathrm{mK}$ despite the heat load from running one or two detectors.

The dilution refrigerator is able to maintain the lowest temperature achieved for long periods of time; in principle forever. Thermometers installed in different stages are read out using four wire measurements and logged on the computer 'Penguin'. The computer 'Penguin' also receives measurements of pressure from different sensors, logs the information and also has software controls for the cryostat.

\subsubsection{Detector mounting}

The detectors are mounted on a copper structure called the 'tower' which is custom built CDMS cold hardware equipment. The tower has different temperature stages that are mechanically connected but thermally insulated [70]. These temperature stages are heat sunk to the different stages of the cryostat. The tower acts as heat sink for signal readout lines from the detectors and also supports signal conditioning electronics (see fig 3.3). The temperature stage on the tower which holds the detectors is connected (physically and thermally) to the base plate thus cooling the detectors to the base plate temperature. 


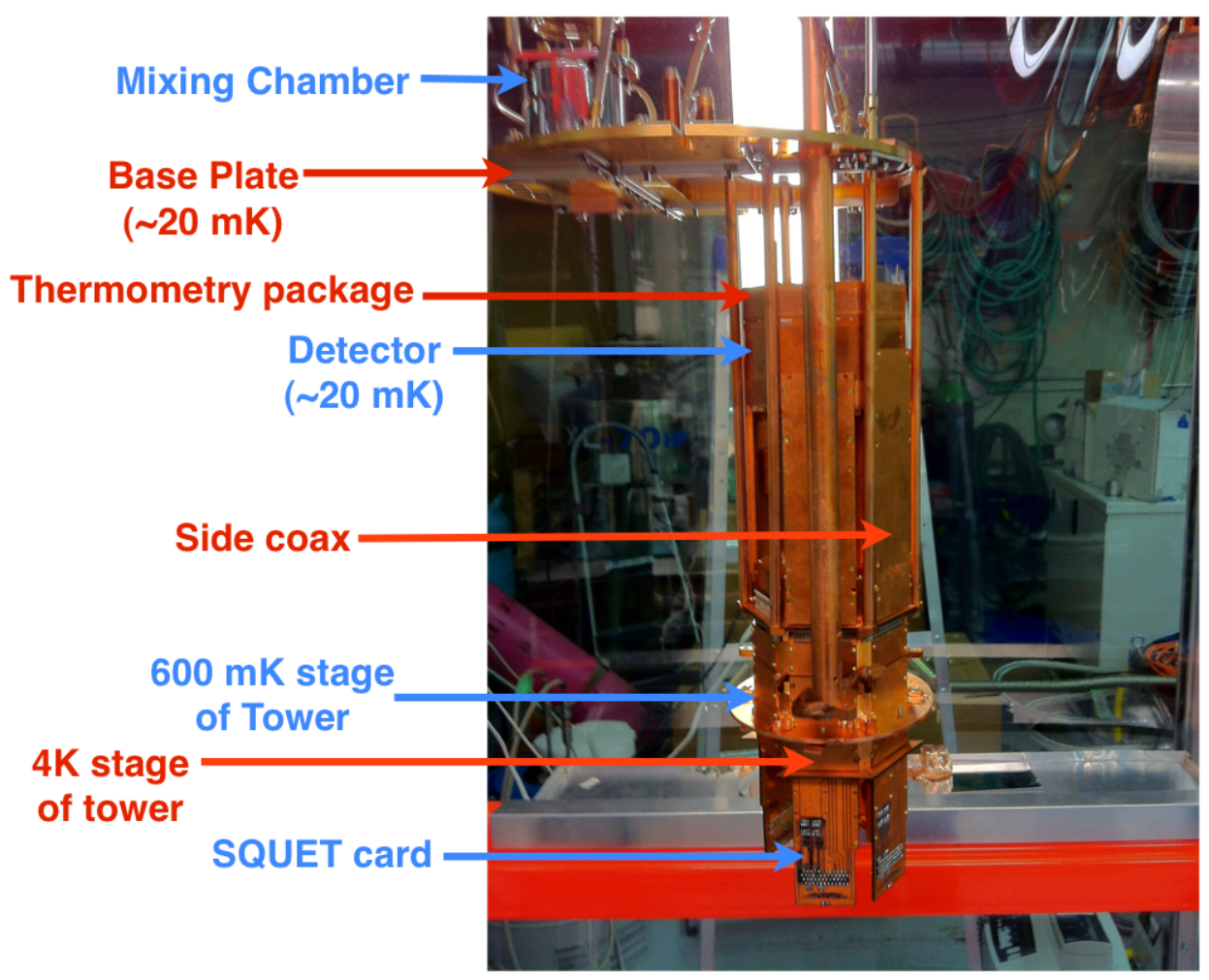

Figure 3.3: Components of the tower. The 'thermometry package' is a casing usually carrying a ruthenium-oxide thermometer and a tower-heater (resistor) and also serves as a holder for small radioactive sources.

\subsubsection{Readout electronics inside the cryostat}

Wires from sensors are connected to copper traces on Detector Interface Boards (DIBs) by wire-bonding. The DIBs are pads made of a cirlex (layered KAPTON) and are attached to the inside of the detector housing (copper enclosure). CDMS custom designed coaxial lines bring the signals from the detector to the $4 \mathrm{~K}$ stage. They are heat sunk at the intermediate temperature stages of the tower. 
At the $4 \mathrm{~K}$ stage these signal lines are connected to SQUET (SQUID and FET) cards (see figure 3.3). The SQUET cards each consist of a FET board and a SQUID board. The FET board is heat-sunk to the $4 \mathrm{~K}$ stage and carries FET based preamplifiers for the two ionization sensor lines. The SQUID board is heat-sunk at the $600 \mathrm{mK}$ stage for better noise performance [70] and carries SQUID based amplifiers for four phonon sensor lines. After preliminary conditioning and amplification, the signals are carried to the room temperature stage (above $70 \mathrm{~K}$ stage) using 'striplines'. The striplines are shielded flex circuits made of copper and KAPTON [70] which terminate on the room temperature end inside the cryostat at 50 pin sub-D connectors. The signals are then brought out of the cryostat through a vacuum bulkhead referred to as the 'e-box' again at 50 pin sub-D connectors.

\subsubsection{Data acquisition system}

\section{Hardware}

The detectors are operated and read out with a 'Detector Control and Readout Card (DCRC) (see figure 3.4 which is directly plugged in to the 50-pin connector at the bulkhead. The DCRC board is a CDMS custom built electronic board with circuits for further signal conditioning, amplification, triggering, digitizing and also acts an interface for the computer where the data is recorded. The DCRC boards also provide a way to bias sensors, send power to LEDs to illuminate detectors (details in section 3.4 and send power down the SQUID arrays to refresh and improve their state. The only connection to the DCRC board is an ethernet cable for data and a power supply at $48 \mathrm{~V}$ through this cable. The DAQ is designed to be dead time free and records the traces every time a trigger condition is satisfied. However there is a problem with 
events being recorded more than once which is discussed in chapter 7. The DAQ software and hardware were built by CDMS as a prototype for the next phase of the experiment and have not been used before to collect data for an analysis of the kind described in this chapter. Hence, we had to address some problems of DAQ software and hardware in order to carry out this work.

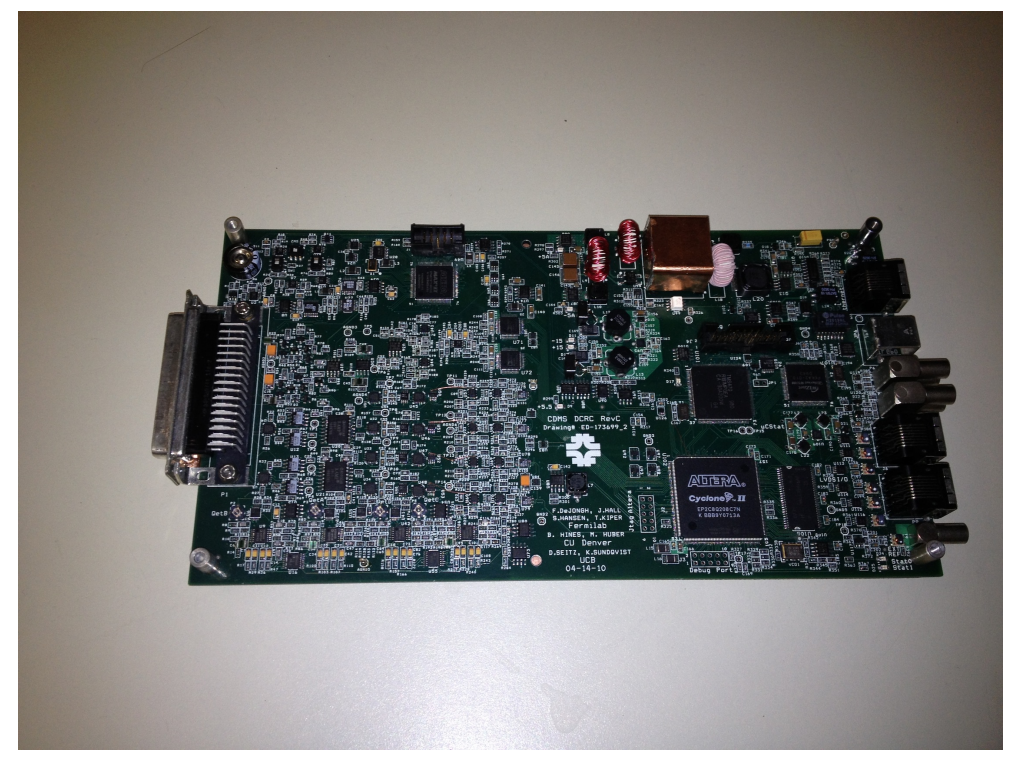

Figure 3.4: DCRC boards are shown plugged to 50 pin sub-D connectors on the e-box.

The cryostat dewar and the mu-metal shield (magnetic shield to deflect earth's magnetic field from SQUIDs in the phonon readout) are inside a permanent cleanroom. Detectors and accompanying hardware that is used inside the cryostat is stored in a nitrogen purge cabinet inside the clean room. This protects copper parts against oxidation and the detectors from radon contamination. 


\section{Software}

The DAQ computer receives and stores the data as binary raw data files. The CDMS test facility DAQ software is a LabVIEW based software package that enables us to interact with the DCRC boards to modify various settings for data acquisition, sensor biasing etc.. They also enable us to see averaged noise spectra and pulses in real time.

\subsection{Data processing and Analysis tools}

The binary raw data files are processed using CDMSBATS which is ROOT based software package written by the CDMS collaboration. It allows data processing algorithms to extract physical quantities from raw data. The resulting information is stored as ROOT files. Another CDMS custom built software package called CAP (CDMS Analysis Package) allows us to load the processed data from ROOT files into MATLAB. CAP also has several scripts which are particularly useful for CDMS/SuperCDMS data analysis.

\subsection{Noise environment}

The noise environment at Queen's test facility is not ideal. The test facility uses a shared lab and does not have special shielding like the main SuperCDMS experiment at Soudan. Apart from the noise that is intrinsic to the readout circuits, our signals also suffer from electromagnetic and mechanically induced (microphonics) noise. The pulse tube cooler with its rotary valve causes pressure oscillations in the pulse tube. Without proper damping these oscillations can set other mechanical components along the signal readout chain to vibrate. If these vibrations vary the impedance of any 
part of the readout circuits they can induce noise in our signals. This is indeed the case as shown in figure 3.5 as an example of the induced noise for channel $Q_{o}$. The noise seems to affect other channels too. There are indications that the alignment of the tower seems to have an effect on the noise. Some contributions to the noise appear to be related to the specific SQUET card used. This is suspected to be coming from mechanical vibrations of components of the SQUET cards that may be loose. We will see in chapter 7 how the poor noise environment raises the energy threshold for our measurement.
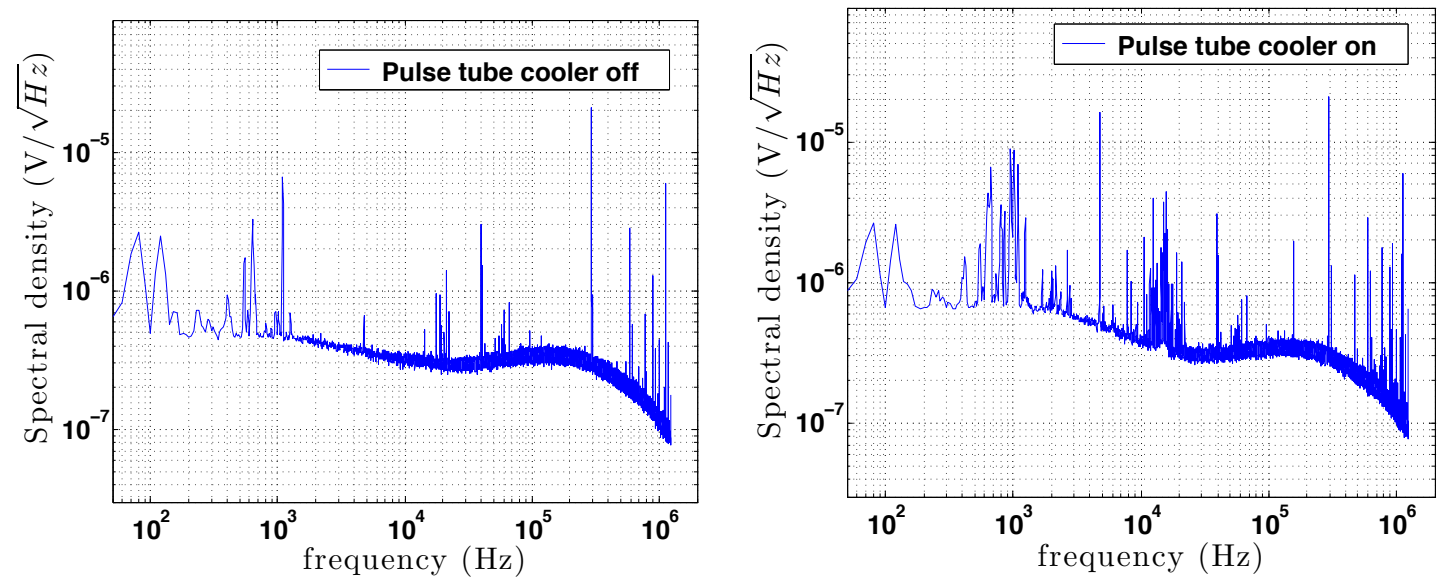

Figure 3.5: Noise spectral density in $Q_{o}$ channel with pulse tube cooler 'OFF' (left plot) and 'ON' (right plot). Additional noise peaks are easily noticed close to $10 \mathrm{kHz}$ when the pulse tube cooler is ' $\mathrm{ON}$ '.

\subsection{Detector de-neutralization}

Our detectors suffer from degradation of ionization signal with time. When taking calibration data the typical duration for degradation is about 5-10 minutes at surface 
test facilities. When taking WIMP search data at the experimental site at Soudan, the time scale is about 3 hours due to the low event rate. The degradation is known to arise from a few different effects. Charge trapping can happen in the bulk due to impurities in the crystal causing signal degradation [74]. The process of the crystal loosing neutrality is referred to as de-neutralization in the SuperCDMS collaboration.

We observed rapid de-neutralization in our detectors operated at theQueen's test facility. After some investigation there was enough indication that IR radiation was reaching our detectors. As described earlier, there are radiation shields down to the $600 \mathrm{mK}$ stage. However, the detectors are exposed to the $600 \mathrm{mK}$ can which has a reflective surface. Although most of the radiation from the $600 \mathrm{mK}$ can carries photons of smaller energies than that required to create an electron hole pair in germanium, if there is radiation from higher temperature stages that leaks into the $600 \mathrm{mK}$ volume, it could impinge on the detectors and release charge carriers. If this is indeed the case, we believe the effect should be reduced by putting absorptive material on the inside of the $600 \mathrm{mK}$ can. An aluminium sheet with an absorptive black coating was used to completely cover the inside surface of the $600 \mathrm{mK}$ can. The black aluminium sheet was chosen for its thermal conductivity (required for cool down to $600 \mathrm{mK}$ ) and ease of application. The sheet was secured to the $600 \mathrm{mK}$ can using copper tape. A skirt of the absorptive material was made around the 600 $\mathrm{mK}$ stage of tower to ensure any radiation from below is absorbed. Figure 3.6 shows the inside of the $600 \mathrm{mK}$ can covered with the black absorptive sheet. This indeed increased the time scale for degradation by a noticeable amount. However we still observed polarity dependent degradation. Charge sensors collecting electrons exhibit degradation sooner than the sensors collecting holes. By looking at the $122 \mathrm{keV}$ line 
from a ${ }^{5} 7 \mathrm{Co}$ source, the time scale for degradation seemed to be about one minute for sensors collecting electrons and at least two minutes for sensors collecting holes.

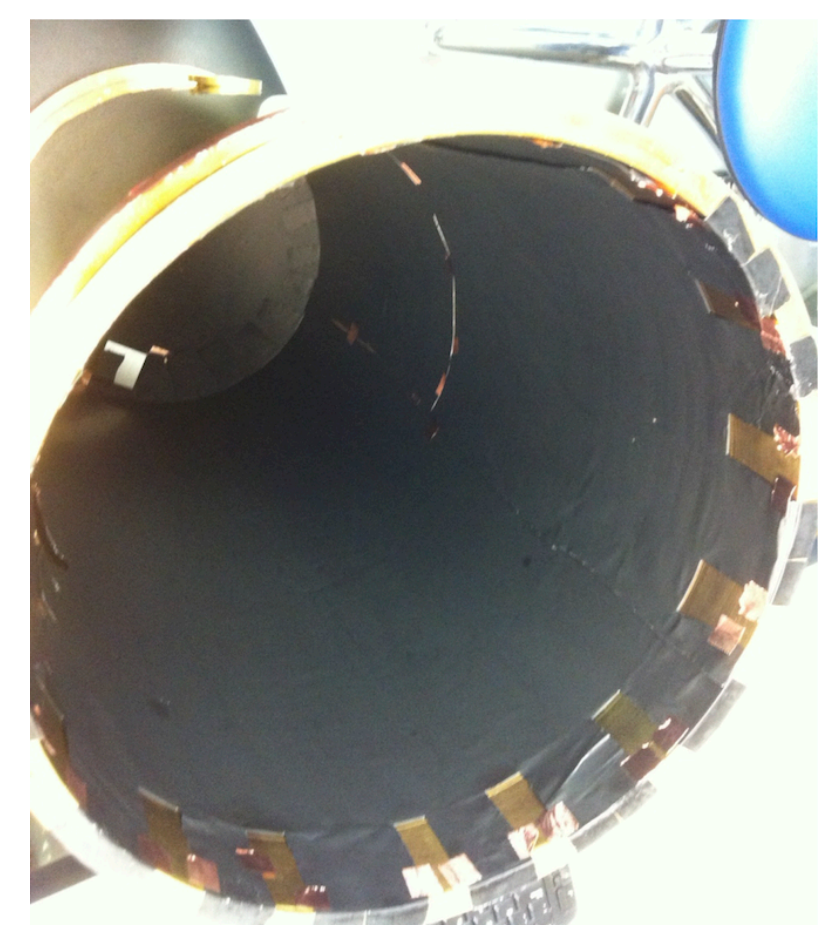

Figure 3.6: Black aluminium sheet applied on the inside of the $600 \mathrm{mK}$ can.

For the study of HiZIP detector surface event discrimination described in chapter 7 we set a restriction on data quality based on the lowest time scale for degradation seen of any channel. Shining LED light on the detector (flashing) for a short duration while all electrodes are grounded releases trapped charges. This is a common procedure adopted by the SuperCDMS collaboration to refresh the charge neutral state of detectors [74]. The test facility DAQ allows us to automate data taking with interspersed flashing of LED light to refresh the detectors. However, flashing LED photons on the detector raises their temperature. The DAQ allows us to automate 
the process of data taking with intermediate flashing and a subsequent wait time for cool down. The downside is that for 24 hours of running in the automated mode we are only able to collect an hour of data. Recent work shows that the neutralization of the detector with the use of an IR shield (copper can) around the tower itself seems to improve neutralization significantly 75$]$. 


\section{Chapter 4}

\section{Fabrication of a ${ }^{210} \mathrm{~Pb}$ Beta Source}

\subsection{Motivation}

In order to assess the surface event discrimination of our detectors we need to take data sets which have a large number of surface events. Low energy betas produce surface events in our detectors and are a major source of background for the experiment. A significant amount of surface event background in our detectors comes from ${ }^{210} \mathrm{~Pb}$ and its daughters. They are usually absorbed within a millimeter of the detector surface which is in the regime of the surface field of the iZIP class of detectors. ${ }^{109} \mathrm{Cd}$ and ${ }^{210} \mathrm{~Pb}$ are commonly used as beta-sources to study surface events in CDMS detectors. We wanted to irradiate the detector surface uniformly with betas and the best option seemed to be to produce a ${ }^{210} \mathrm{~Pb}$ source which spanned the surface and could be fitted easily to the detector assembly. We needed a source which would produce about 2030 events per second in our detector which is sufficiently above the background rate without creating many pileup events. The following sections describe the fabrication of a ${ }^{210} \mathrm{~Pb}$ source. 


\subsection{Principle and Design}

${ }^{210} \mathrm{~Pb}$ is a relatively long-lived isotope in the ${ }^{222} \mathrm{Rn}$ decay chain. The radon decay chain is shown in figure 4.1 .

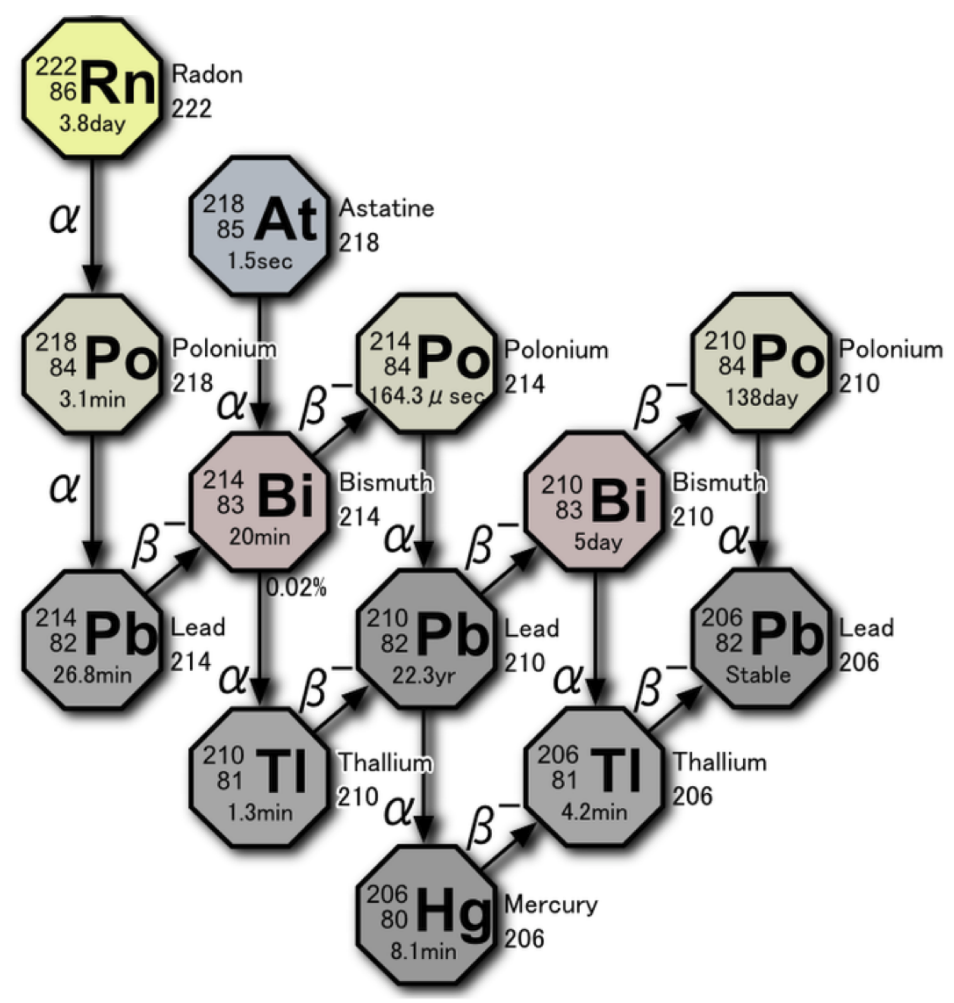

Figure 4.1: The radon decay chain. Half lives are written below the isotopes chemical symbol. The transitions ${ }^{210} \mathrm{~Pb} \rightarrow{ }^{206} \mathrm{Hg}$ and ${ }^{210} \mathrm{Bi} \rightarrow{ }^{206} \mathrm{Tl}$ have very small probabilities of $2 \times 10^{-6} \%$ and $1.3 \times 10^{-4} \%$ and can be neglected for our purposes. Figure from http://www.test.org/doe/.

${ }^{222} \mathrm{Rn}$ has a half life of about 3.8 days and decays to ${ }^{218} \mathrm{Po}$ through $\alpha$ decay. ${ }^{218} \mathrm{Po}$ and the following isotopes in the chain are relatively short lived until ${ }^{210} \mathrm{~Pb}$ which 
has a half life of about 22.3 years. If we were to start with a certain amount of ${ }^{222} \mathrm{Rn}$ most of it would decay into ${ }^{210} \mathrm{~Pb}$ within $2-3$ weeks. The polonium is mostly positively charged and can be deposited on a surface by applying a voltage to the surface. The Rn daughters thus deposited on surfaces lead to partial implantation due to subsequent alpha-decays. It is possible that if there is dust or moisture around, the ions may stick to dust particles or be lost to the moisture. Hence, having the radon gas inside a clean container free of moisture is essential. A copper plate with the dimensions matching the detector surface was chosen for the deposition. A high voltage of about - $1 \mathrm{kV}$ was used to attract the ions toward this plate.

Given the design of the detector assembly, the copper plate would sit within $\sim 1$ $\mathrm{cm}$ from the detector surface. Given that half of the activity from the plate would be towards the inside of the plate, the maximum activity that is seen by the detector would be $50 \%$ of the total activity. Hence to produce a rate of about $30 \mathrm{~Bq}$ towards the detector, the total activity of the deposited material needs to be about $60 \mathrm{~Bq}$.

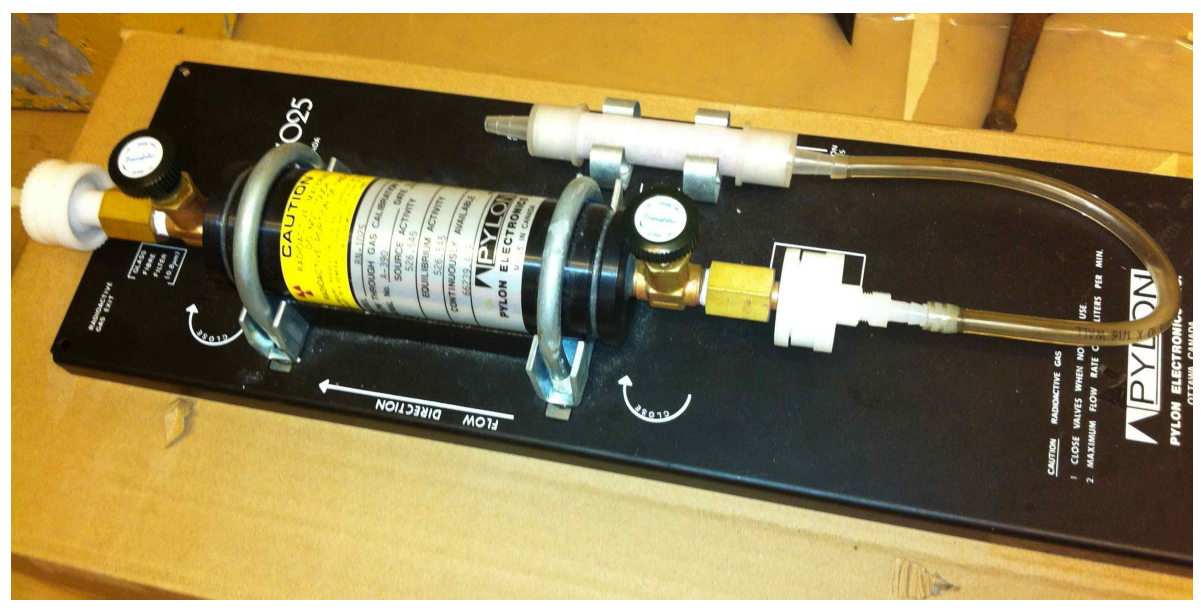

Figure 4.2: Radon source from Pylon (Model RN-1025) 
We used a radon source from Pylon (see figure 4.2) with a calibrated equilibrium radon activity of $526.5 \mathrm{kBq}$. A cylindrical container made of aluminium was designed to hold the radon gas during exposure to the copper plate. The copper plate was exposed to the gas by using it as the lid of this container. In order to apply a high negative voltage to the copper plate with respect to the aluminium container it had to be separated by a gasket which could sustain the high voltage across it. A plastic gasket with grooves on either side for rubber o-rings was fabricated. It was ensured that the thickness of the plastic gasket was enough to sustain the high voltage. A rough estimate of the expected activity from the deposited ${ }^{210} \mathrm{~Pb}$ is calculated below.

The total activity (A) of a given sample of radioactive material is defined by

$$
\mathrm{A}=-\frac{\mathrm{dN}}{\mathrm{dt}}=\lambda \mathrm{N}
$$

where $\mathrm{N}$ is the total number of nuclei, $\mathrm{t}$ is time, and $\lambda$ is the decay constant for the given nucleus. The decay constant can be expressed in terms of the half life $\left(t_{1 / 2}\right)$ of the given nuclei as in equation 4.2 .

$$
\lambda=\frac{\ln (2)}{\mathrm{t}_{1 / 2}}
$$

Given the activity of $526.5 \mathrm{kBq}$ for the radon source we can calculate the total number of radon atoms, which comes out to be $2.510 \times 10^{11}$. The volume of the aluminium container is roughly equal to the volume of the Pylon source $\left(\sim 115 \mathrm{~cm}^{3}\right)$. Since we could empty the exposure chamber to about 0.2 bar, we could fill about $80 \%$ the volume with radon. There may also be some loss of radon in the source itself due to the volume of the pipe between the radon source and the exposure chamber. Hence, 
assuming we transfer about $50 \%$ of radon from the source into the chamber, with sufficient time for most atoms to decay to ${ }^{210} \mathrm{~Pb}$, we end up with $1.2 \times 10^{11}$ atoms of ${ }^{210} \mathrm{~Pb} .{ }^{210} \mathrm{~Pb}$ with a half life of 22.3 years has a total activity of $\sim 125 \mathrm{~Bq}$ for the given number of lead atoms. Since we don't want our detectors and the detector assembly to be contaminated with ${ }^{210} \mathrm{~Pb}$ we would like to wash away the easily removable radioactivity on the surface. With the polonium deposited on the surface, we can expect about $50 \%$ to be implanted in the copper (recoiling daughter nuclei). Hence, assuming we wash off another $50 \%$ of the activity and only $50 \%$ of the activity form the implanted nuclei is directed towards the detector we end up with $30 \mathrm{~Bq}$ that we require. Section 4.3 below describes the details of the setup used and the procedure followed to produce the source.

\subsection{Experimental setup and Procedure}

The aluminium container was secured to the copper plate with a plastic gasket in between, with rubber o-rings between each other, and is secured with a clamp. This setup is referred to as the 'exposure chamber'. A schematic of the parts of the exposure chamber are shown in figure 4.3. A picture of the exposure chamber secured with the clamp can be seen in figure 4.5 . 


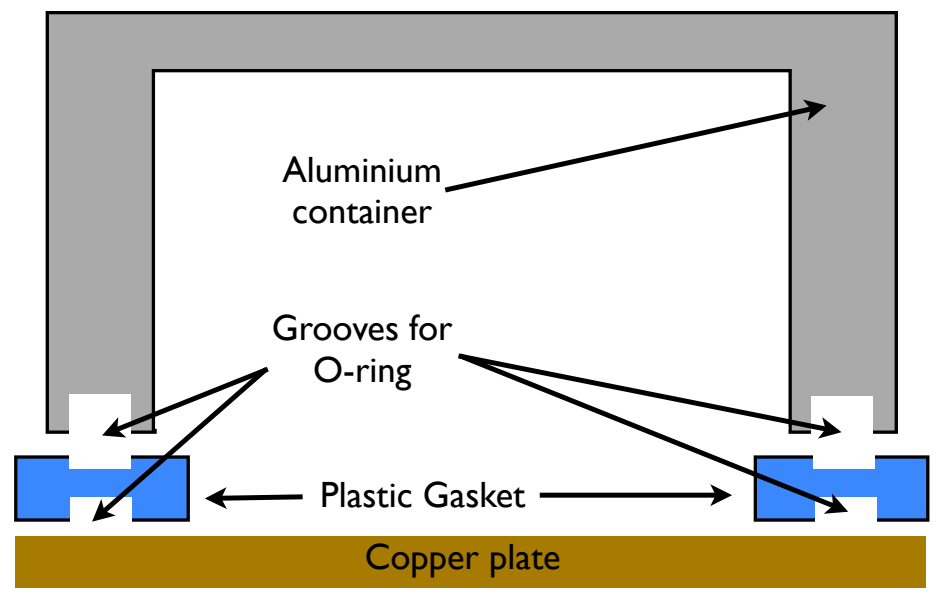

Figure 4.3: A schematic of the parts of the 'exposure chamber'. It consists of an aluminium container with a plastic gasket and a copper plate with grooves for o-rings.

The apparatus used to produce the lead source is shown in figure 4.4. As described before the aluminium container was made such that the volume was roughly the same as the pylon source. Copper strips were held pressed against the copper plate and the aluminium box to connect them to the high-voltage (HV) supply. 


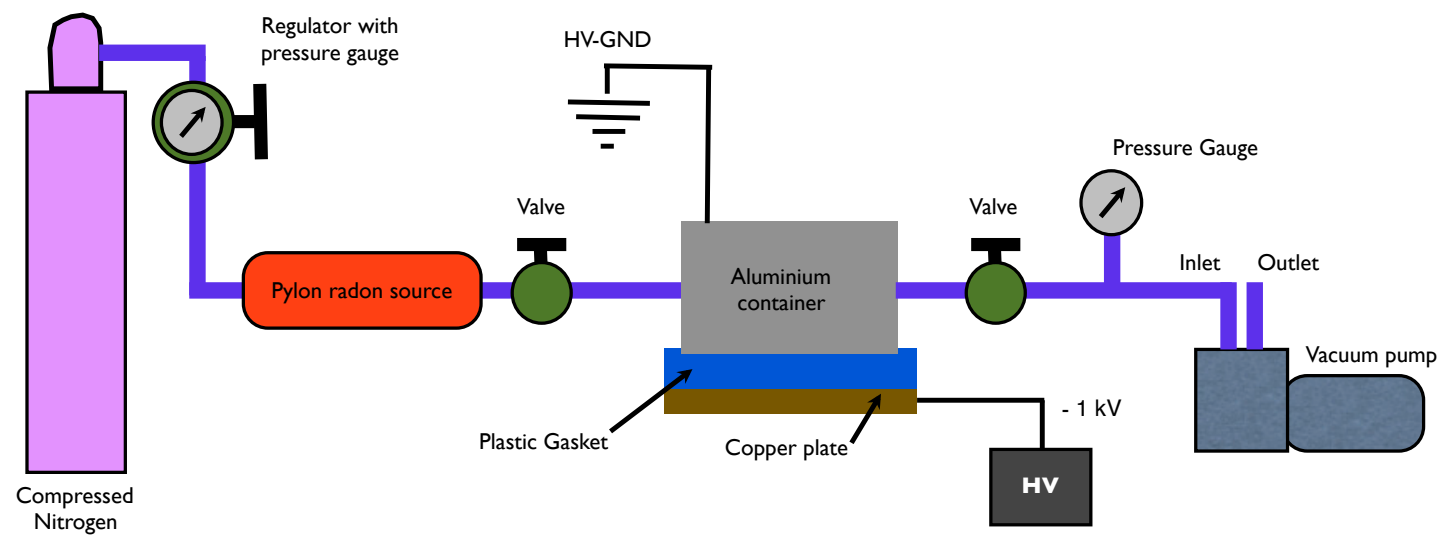

Figure 4.4: A schematic of the experimental setup used for radon exposure of the copper plate.

A compressed nitrogen cylinder was connected to the back of the source to flush the source. A vacuum pump was connected to the aluminium box along with a vacuum gauge to monitor the vacuum during pumping. A standalone HV power supply module was used to provide the $-1 \mathrm{kV}$ to the plate. Rubber o-rings were placed into the grooves made on the aluminium box and the plastic gasket. A few tests were done to see if the aluminium container held pressed against the copper plate could sustain vacuum once the container was closed off to the vacuum pump. Tests were done to see how to best secure the clamps. With this setup and the available pump we reached an absolute pressure of roughly 0.2 bar. The whole setup was tested for leaks and cleaned. It was then ready for the radon exposure. The copper plate was specially cleaned with bright-dip (a mixture of $\mathrm{HCl}, \mathrm{HNO}_{3}$ and $\mathrm{H}_{2} \mathrm{SO}_{4}$ ), then washed in de-ionized water and wiped dry before using it in the setup. 


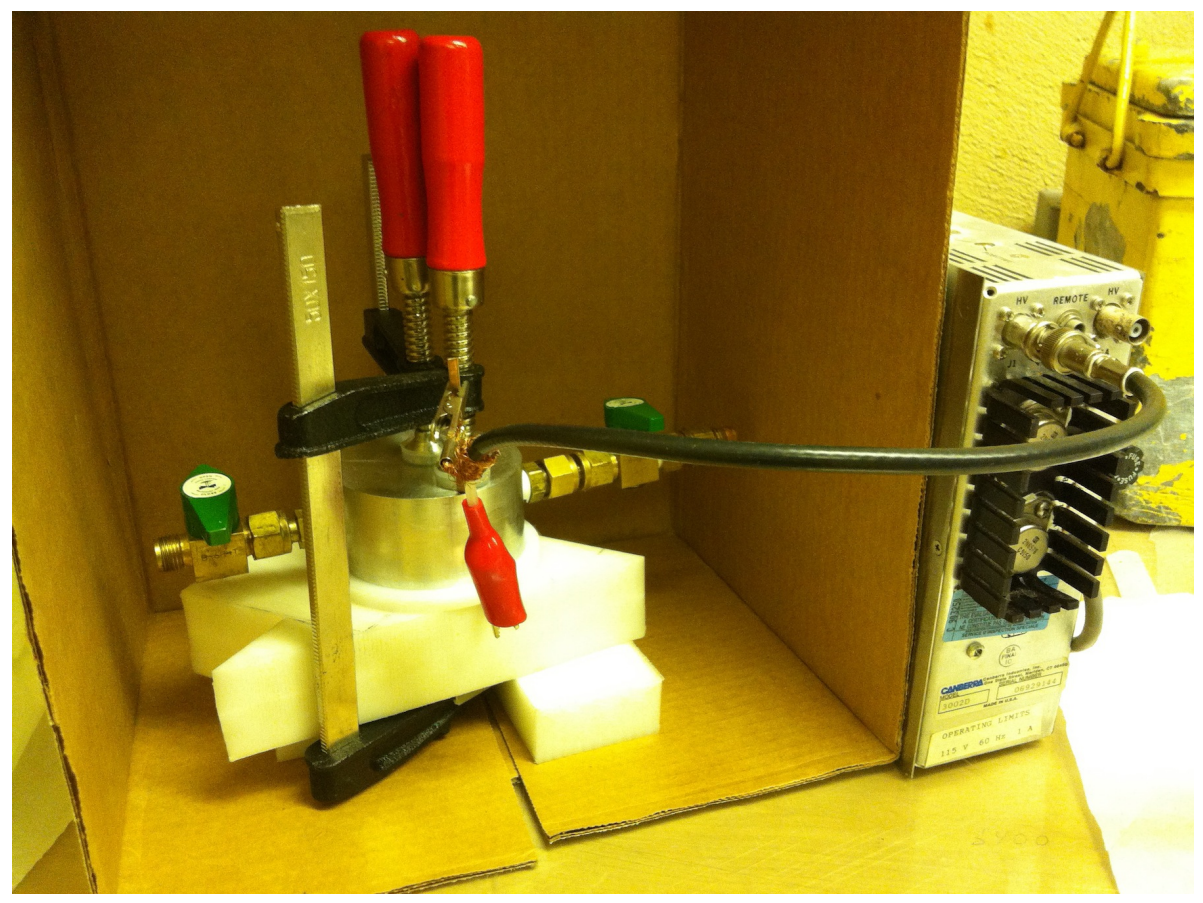

Figure 4.5: The exposure chamber connected to HV supply (the rest of the apparatus is removed).

After the apparatus was set up, the exposure chamber (the aluminium box with the copper plate) was emptied using the pump. Radon from the source was let into the exposure chamber allowing nitrogen to come in to the source. After a few seconds, the source and the exposure chamber were closed off. The connections to the pump and the source were removed and the HV was connected to the copper plate (see figure 4.5). 


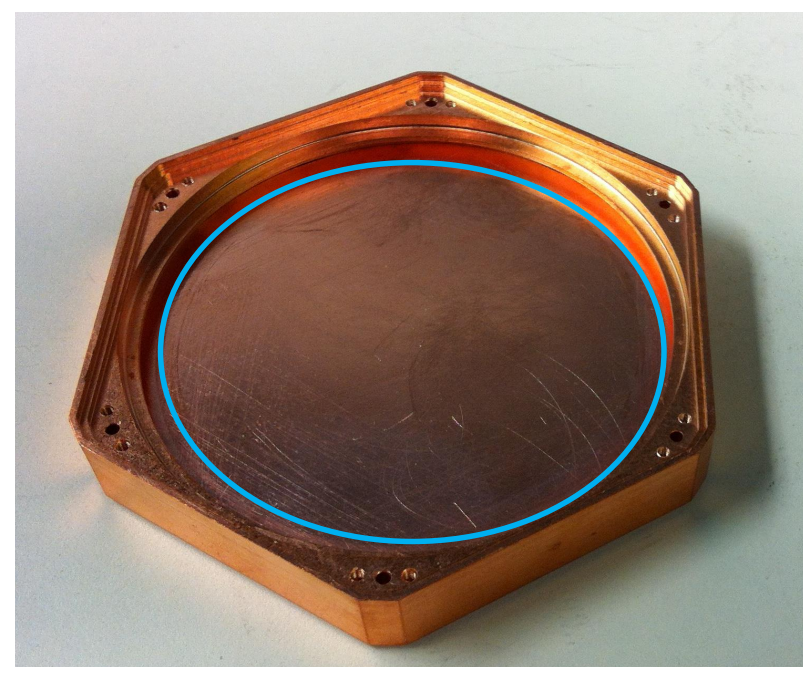

Figure 4.6: The finished ${ }^{210} \mathrm{~Pb}$ source. The copper plate is secured in a source holder which can be mounted either directly above or below the detector. The active area is within the blue circle.

After 20 days, the apparatus was dismantled and the copper plate was removed. A Geiger counter was used to measure the activity from the plate and compared to the background activity. We used a radiation monitor (Geiger counter) to confirm a slightly elevated activity on the exposed copper surface while no significant activation was observed on the inside of the aluminum container. We cleaned the source with water and dried the surface with a wipe. The activity on the plate was checked again with the Geiger counter and found it similar to before washing. A picture of the finished ${ }^{210} \mathrm{~Pb}$ source plate in a holder is seen in figure 4.6. The observed event rate from the source when mounted next to the test detector (see chapter 7) was roughly $20 \mathrm{~Hz}$ in accordance with our expectation. 


\section{Chapter 5}

\section{The HiZIP and Charge Noise}

\section{Studies}

Here we propose an alternate detector design called the HiZIP. The HiZIP is a modified version of the iZIP detector mentioned in chapter 2. The following section describes the HiZIP design and the motivation for its development. Section 5.2 discusses analytical calculations done to estimate the observed electronic noise in charge channels. It also estimates the contribution of crosstalk noise due to capacitive coupling between charge channels in the HiZIP detector. Section 5.3 serves to introduce surface event discrimination studies of the HiZIP detector which are discussed in greater detail in chapter 6. Section 5.4 serves as an introduction to the surface event discrimination studies of a real HiZIP device operated at Queen's Test Facility. It also discusses the performance of a new charge channel that is created in the HiZIP design. 


\subsection{The HiZIP: concept and motivation}

As described in chapter 2, the iZIP detector has four phonon sensors and two charge sensors on each side. The interleaved charge and ground electrodes allow us to reject surface events with great efficiency. The HiZIP was conceptualized as a detector with reduced complexity which would in turn reduce the complexity and quantity of data and hence the analysis effort. Another important motivation for the HiZIP design is the possibility to detect and discriminate double scatter event close to the surfaces which can be mistaken for a WIMP. For example from a photon Compton scattering close to the surface on each side, it would produce a charge signal on both side 1 and 2 and hence mimic a bulk event (see figure 5.1). Due to reduced ionization collection [69] for events close to the surfaces these double surface scatter events are a source of background for our experiment. These events are referred to as 'Multiple Interaction Surface Scatter' (MISS) events. 


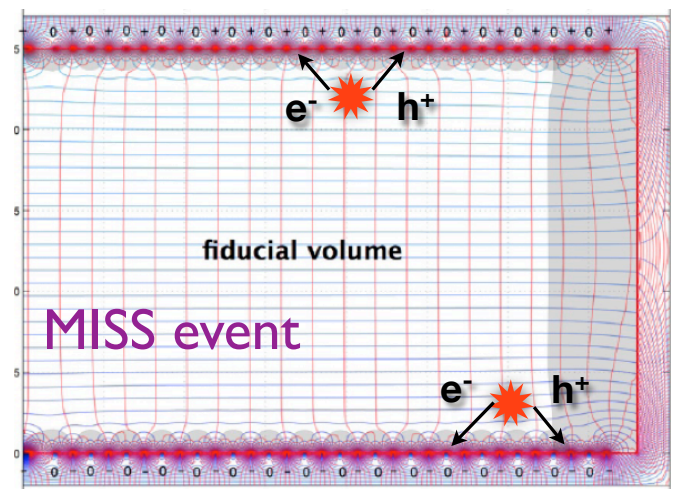

Figure 5.1: A MISS event in an iZIP detector caused by a double scatter event close to side 1 and 2 surfaces. This results in a charge collection on the charge sensors on both sides and hence mimics a bulk event. Owing to these interactions happening close to the surface, they often result in incomplete charge collection and hence become background for WIMP detection.

Studies to discriminate against MISS events using differences in pulse shapes compared to single interactions have yielded promising results [76] [77]. Another way to identify MISS events could be imagined if we were able to measure the amount of charge collected at the ground electrodes; in fact, it would be sufficient to measure the charge on one of the ground electrodes since MISS events lead to charge collection on the ground electrodes on both sides of the detector. The HiZIP detector design allows us to do exactly this. The HiZIP has phonon sensors on only one side. It has four charge channels in total just like the iZIP, but the interleaved phonon sensors on the other side are replaced by a new charge sensor $\mathrm{Q}_{p}$ (see figure 5.2). 

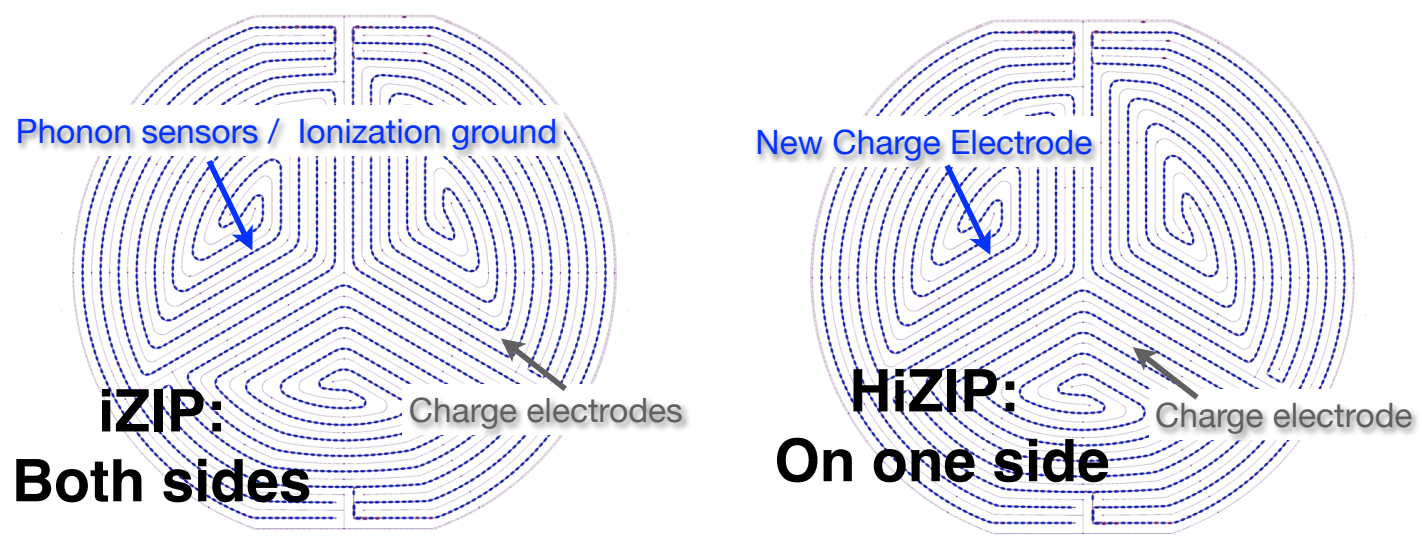

Figure 5.2: The iZIP (left) has charge sensors interleaved with phonon sensors on both sides. The phonon sensors act as ground reference for charge measurement. The HiZIP (right) has a new charge electrode on one side in place of the phonon sensors. Also, the iZIP has $Q_{\text {inner }}$ and Q $_{\text {outer }}$ electrodes on both sides, while the HiZIP has them combined on one side and separate on the other. This is due to the constraint of four charge channels per iZIP from the existing readout electronics. Note that the $\mathrm{Q}_{\text {inner }} / \mathrm{Q}_{\text {outer }}$ distinction is not made explicit here, but can be seen in figure 5.3 .

Due to constraints from the existing readout electronics we can only have four charge channels in total for each iZIP type detector. Given this constraint, the two possible configurations for wiring the HiZIP charge sensors are shown below ( see figure 5.3). 

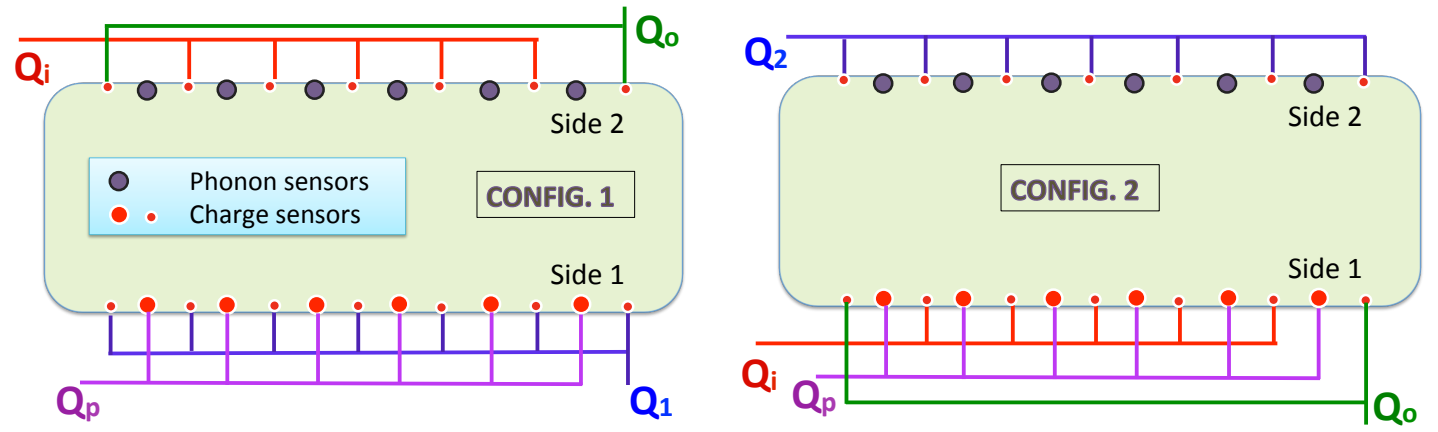

Figure 5.3: HiZIP charge sensor wiring configuration 1 (left) and configuration 2 (right). HiZIP config. 1 has two charge sensors per side with Qinner

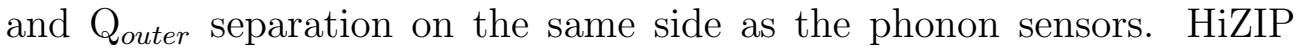
config. 2 has three charge sensors on the side opposite to phonon sensor face and only one on the same side as the phonon sensors. Note that since the iZIP is in fact a symmetric device, the sensor wiring scheme for HiZIP side 1 shown here can equally well be implemented on side 2 .

Since we can read out the charge on both the interleaved electrodes on side 1 in either of the HiZIP designs in figure 5.3, we can determine with certainty if any interaction happened close to the side 1 surface. Consequently we can also differentiate a bulk event from a MISS event. MISS events are too rare to be a concern for SuperCDMS Soudan [78], but if we get into the ton-year range, as foreseen for the SNOLAB phase of the experiment, we will have to start worrying about them. If we are able to achieve a surface event discrimination for the HiZIP that is comparable to that of the iZIP with the added advantages mentioned above, we believe the HiZIP will be an attractive option for dark matter detection.

In order to test the viability of a HiZIP device many studies were done by the SuperCDMS group at Queen's. One of the studies estimates the expected level of 
electronic noise in the charge readout circuits and is discussed in detail in section 5.2 . Section 5.3 introduces the HiZIP studies carried out using data taken with an iZIP detector, which are discussed in greater detail in chapter 6. Section 5.4 introduces HiZIP studies carried out using a real HiZIP device; the G16K detector, which was operated at Queen's test facility to take data. Chapter 7 on the G16K HiZIP detector explains the various aspects of the experiment, analysis and findings in detail.

\subsection{Electronic noise in charge readout circuit}

The ionization (charge) readout circuit is shown in figure 5.4 . The sensor capacitance $C_{d}$ for any charge channel is the equivalent capacitance between the channel's bias electrode and ground. For a crystal alone, without any metallic housing, $\mathrm{C}_{d}$ of a charge sensor on a given side is the combination of capacitance to the ground electrode on the same side of the crystal in parallel with the capacitance to the ground electrode on the other side. Since the capacitance to the ground electrode on the other side is relatively small compared to that on the same side [79], $\mathrm{C}_{d}$ for $\mathrm{Q}_{\text {inner }}$ or $\mathrm{Q}_{\text {outer }}$ on a given side for an iZIP could be approximated by the ecapacitance to the ground electrode on the same side alone. 


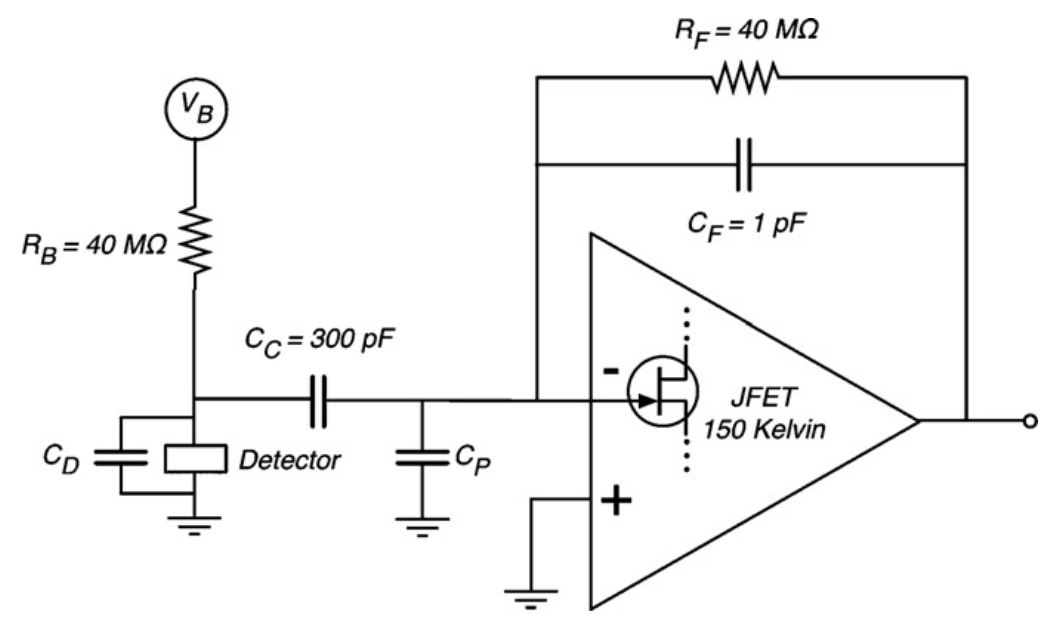

Figure 5.4: Charge readout circuit for SuperCDMS detectors. The different components are: sensor capacitance $\left(C_{d}\right)$, feedback capacitance $\left(C_{f}\right)$, operational-amplifier (op-amp) gate capacitance $\left(C_{p}\right)$, feedback resistance $\left(R_{f}\right)$, bias resistance $\left(R_{b}\right)$ and coupling capacitance $\left(C_{c}\right)$. Figure from 70

However, in the case of a HiZIP, instrumenting a charge readout to the interleaved ground electrode on a given side means that $\mathrm{C}_{d}$ for all the charge sensors on this side can be approximated by the capacitance of the sensor to the ground electrode on the other side alone. The new interleaved charge electrode in the HiZIP $\left(\mathrm{Q}_{p}\right.$ in figure 5.3) also has a strong capacitive coupling to other charge electrodes on the same side $\left(\mathrm{Q}_{1}\right.$ in figure 5.3 (left), and $\mathrm{Q}_{\text {inner }}$ and $\mathrm{Q}_{\text {outer }}$ in figure 5.3 (right)). The charge channels on this side have higher crosstalk between each other. This crosstalk may affect our energy estimation of a signal pulse as well as the noise threshold for the measurement. To get an idea of the coupling between the channels we calculate and compare the current transfer function for a detector current in a given sensor (channel 
transfer function, CTF) with that of a detector current in an adjacent sensor that is capacitively coupled (crosstalk transfer function, XTF). The current transfer function (also known as transfer impedance) for the charge readout circuit is defined as the change in the voltage $(\mathrm{V})$ of the op-amp for a unit change in the input current (I) by the detector (i.e. $\delta \mathrm{V} / \delta \mathrm{I}$ ) expressed in the Laplace domain as a function of frequency.

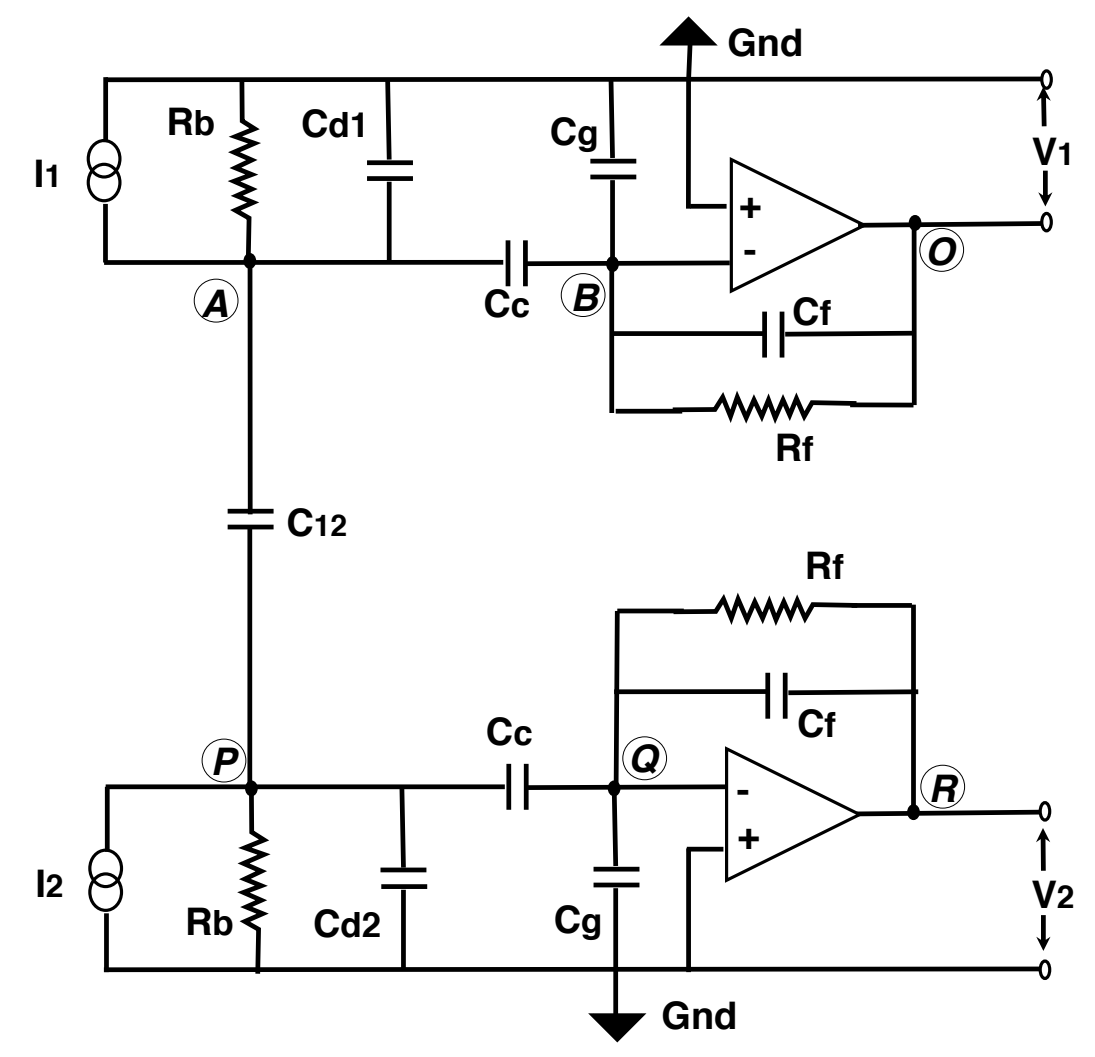

Figure 5.5: Charge readout circuits of two capacitively coupled charge sensors.

Figure 5.5 shows the charge readout circuits of two capacitively coupled channels. $\mathrm{C}_{d 1}$ and $\mathrm{C}_{d 2}$ are the sensor 1 and sensor 2 capacitances to the ground electrode. Similarly $\mathrm{I}_{1}, \mathrm{I}_{2}$ and $\mathrm{V}_{1}$ and $\mathrm{V}_{2}$ are the detector currents and output voltages respectively for sensor 1 and sensor 2 circuits. $\mathrm{C}_{12}$ is the mutual capacitance of the two sensors. 
The remaining circuit components are the same in both circuits since the same readout electronics are used for all charge channels. The parasitic capacitance of figure 5.4 will be referred to as the gate capacitance $\left(\mathrm{C}_{g}\right)$. In this case, we have two 'channel transfer functions' and two 'crosstalk transfer functions'. These can be represented as $\delta \mathrm{V}_{i} / \delta \mathrm{I}_{j}$, where the subscript indices $i$ and $j$ can take values 1 and 2 corresponding to sensors 1 and 2 .

In the case of an iZIP detector the capacitive coupling is expected be maximal between $\mathrm{Q}_{\text {inner }}\left(\mathrm{Q}_{i}\right)$ and $\mathrm{Q}_{\text {outer }}\left(\mathrm{Q}_{o}\right)$ sensors on the same side of the detector. Since $\mathrm{Q}_{\text {inner }}$ is our charge sensor of interest, we calculate here, the CTF of $\mathrm{Q}_{\text {inner }}$ and XTF of $\mathrm{Q}_{\text {inner }}$ with regard to $\mathrm{Q}_{\text {outer }}$ (for short: $\mathrm{Q}_{i}-\mathrm{Q}_{o}$ ). If sensor 1 is chosen to be $\mathrm{Q}_{\text {inner }}$ and sensor 2 is $\mathrm{Q}_{\text {outer }}$, the $\mathrm{CTF}$ for $\mathrm{Q}_{\text {inner }}$ is given by $\delta \mathrm{V}_{1} / \delta \mathrm{I}_{1}$ and the XTF for Q $\delta \mathrm{V}_{Q i} / \delta \mathrm{I}_{Q o}$ respectively. Let us also represent the $\mathrm{Q}_{i}$ sensor capacitance as $\mathrm{C}_{d Q i}$, $\mathrm{Q}_{o}$ sensor capacitance as $\mathrm{C}_{d Q o}$ and the capacitance between $\mathrm{Q}_{i}$ and $\mathrm{Q}_{o}$ as $\mathrm{C}_{Q i Q o}$.

Figure 5.6 shows the circuit used to derive the CTF for $Q_{i}$. The current $\mathrm{I}_{Q i}$ sees the net impedance $\mathrm{Z}_{Q o}$ of the $\mathrm{Q}_{\text {outer }}$ sensor circuit (including $\mathrm{C}_{Q i Q o}$ ) to be parallel to $\mathrm{R}_{b}$ and $\mathrm{C}_{d Q i}$. We estimate the impedance $\mathrm{Z}_{Q o}$ by the steady state impedance of the $Q_{o}$ amplifier circuit. In a steady state the output voltage of the amplifier is zero and hence can be assumed to be grounded (see figure 5.7). The net impedance between nodes $R$ and $A$ is then $\mathrm{Z}_{Q_{o}}$. For a conventional iZIP detector the $\mathrm{Q}_{i}$ and $\mathrm{Q}_{o}$ sensors have capacitances $C_{d Q i}=57 \mathrm{pF}$ and $C_{d Q o}=24 \mathrm{pF}$ to ground electrode respectively, while the channel coupling $\mathrm{C}_{Q i Q o}=3 \mathrm{pF}[79$. These values of capacitances have been calculated from finite element simulations carrier out by a collaborator (Scott Hertel) 79$]$. 


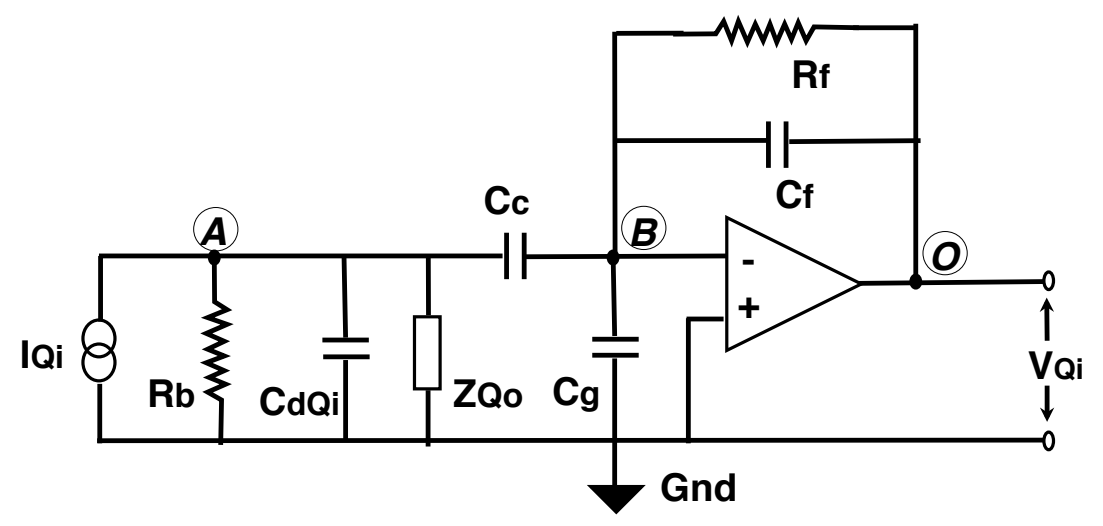

Figure 5.6: Qinner charge readout circuit with the net impedance of Qouter sensor circuit in parallel with $\mathrm{C}_{d Q i}$.

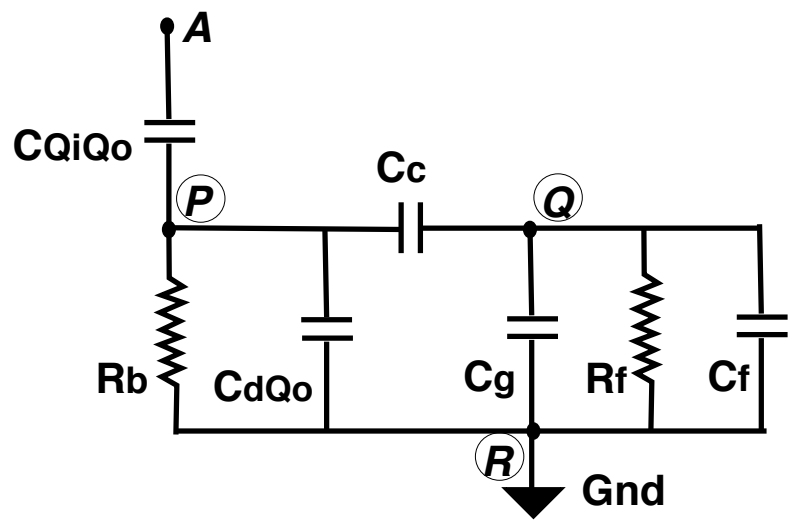

Figure 5.7: Equivalent circuit used to calculate $\mathrm{Z}_{Q o}$.

The voltage transfer function of an op-amp circuit is the ratio of the output voltage to the gate voltage, i.e. the voltage at node B (see figure 5.6) and is given by

$$
\mathrm{H}(\mathrm{s})=\frac{\alpha(\mathrm{s})}{1+\alpha(\mathrm{s}) \beta(\mathrm{s})}
$$


where $\alpha(s)$ is the open-loop gain and the product $\alpha(s) \beta(s)$ is the loop gain of the amplifier. The open loop gain is calculated using the op-amp's parameters. The feedback factor $\beta(s)$ is the fraction of output voltage fed back to the amplifier gate. The CTF is then obtained by the product of the net impedance to ground seen by the current $\mathrm{I}_{Q i}$ with the voltage transfer function $\mathrm{H}(\mathrm{s})$. This net impedance to ground is the impedance across which the current $\mathrm{I}_{Q i}$ flows to produce the voltage drop at the gate of the op-amp.

In order to calculate the XTF we follow a similar methodology. The output of the amplifier in the $\mathrm{Q}_{o}$ sensor circuit is again considered to be held to ground and the voltage drop produced by $\mathrm{I}_{Q_{o}}$ at the gate of the op-amp in $\mathrm{Q}_{i}$ circuit is determined. The product this voltage drop with $\mathrm{H}(\mathrm{s})$ of the $\mathrm{Q}_{i}$ circuit gives us the crosstalk transfer function XTF. Details of these calculations are available in appendix A. Figure 5.8 shows the CTF and XTF for the $\mathrm{Q}_{i}$ channel. In the plateau region of the transfer functions it can be seen that the crosstalk transimpedance is $\sim 3-5 \%$ of the channel transimpedance. The observed signal crosstalk between $\mathrm{Q}_{\text {inner }}$ and $\mathrm{Q}_{\text {outer }}$ is also found to be consistent with this prediction [63] [80]. 


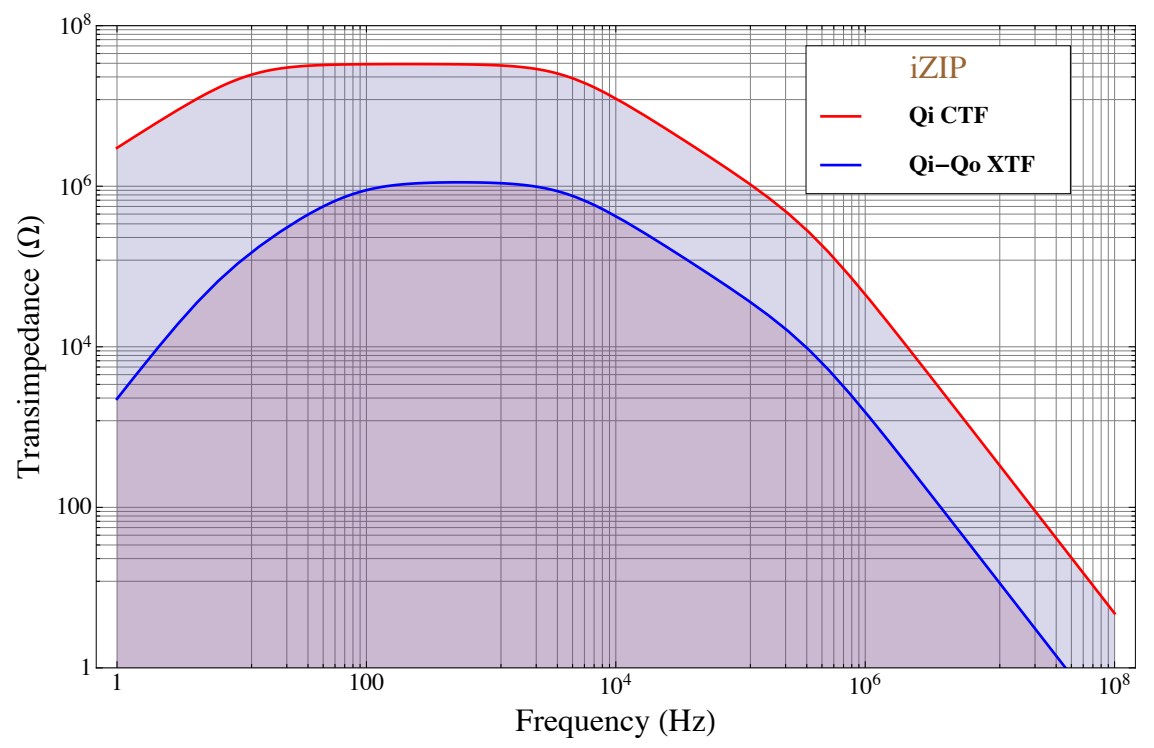

Figure 5.8: $\mathrm{Q}_{i}$ channel and crosstalk transfer functions for iZIP.

The CTF and XTF for $\mathrm{Q}_{p}$ in HiZIP configuration 1 are calculated in the same way considering the coupled circuit to be that of $\mathrm{Q}_{1}$ (see figure 5.9). The CTF for $\mathrm{Q}_{p}$ is given by $\delta \mathrm{V}_{Q p} / \delta \mathrm{I}_{Q p}$ and the $\mathrm{XTF}$ for $Q_{p}-Q_{1}$ is given by $\delta \mathrm{V}_{Q p} / \delta \mathrm{I}_{Q 1}$. Since $\mathrm{Q}_{1}$ and $\mathrm{Q}_{p}$ in this configuration are sensors that are interleaved and span the entire side 2 surface they have been assumed to have identical capacitances to ground (i.e. $\mathrm{C}_{d 1}$ $\left.=\mathrm{C}_{d p}=5 \mathrm{pF}\right)$, although in reality $\mathrm{Q}_{p}$ may have a somewhat higher capacitance due to its greater surface area. The coupling between the channels is $C_{1 p}=81 \mathrm{pF}[79$. 


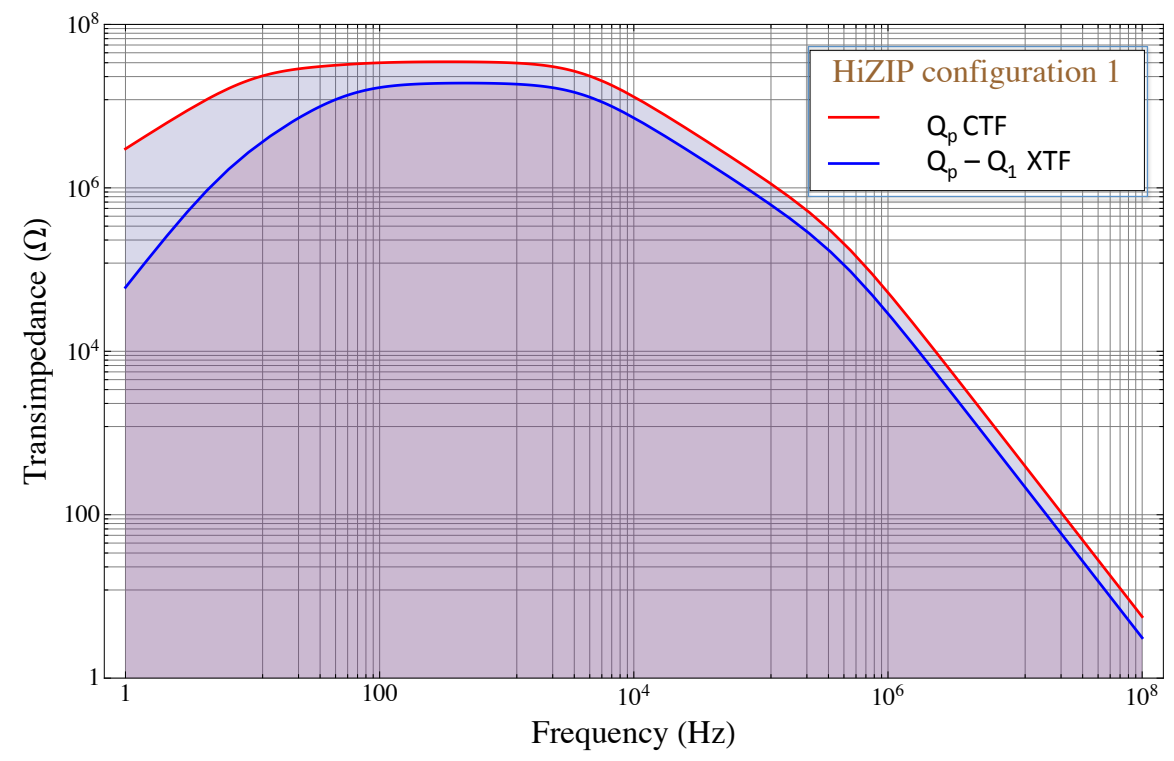

Figure 5.9: $\mathrm{Q}_{p}$ channel and crosstalk transfer functions for HiZIP configuration 1.

In configuration 2 of the HiZIP the capacitive coupling is strong between $Q_{p}$, $Q_{\text {inner }}\left(Q_{i}\right)$ and $Q_{\text {outer }}\left(Q_{o}\right)$. Figure 5.10 shows CTF for $Q_{p}$ and the two XTFs corresponding to crosstalk from $Q_{i}$ and $Q_{o}$. This result makes sense since we expect a greater crosstalk in $\mathrm{Q}_{p}$ from $\mathrm{Q}_{i}$ than from $\mathrm{Q}_{o}$ due to stronger coupling between $Q_{p}$ from $Q_{i}$. The sensor capacitances for $Q_{p}, Q_{i}$ and $Q_{o}$ are $C_{d Q p}=5 \mathrm{pF}, C_{d Q i}=3 \mathrm{pF}$ and $C_{d Q o}=1.6 \mathrm{pF}$. The coupling between $Q_{p^{-}} Q_{i}$ and $Q_{p^{-}} Q_{o}$ are $C_{Q p Q i}=57 \mathrm{pF}$ and $C_{Q p Q_{o}}=24 \mathrm{pF}[79]$. 


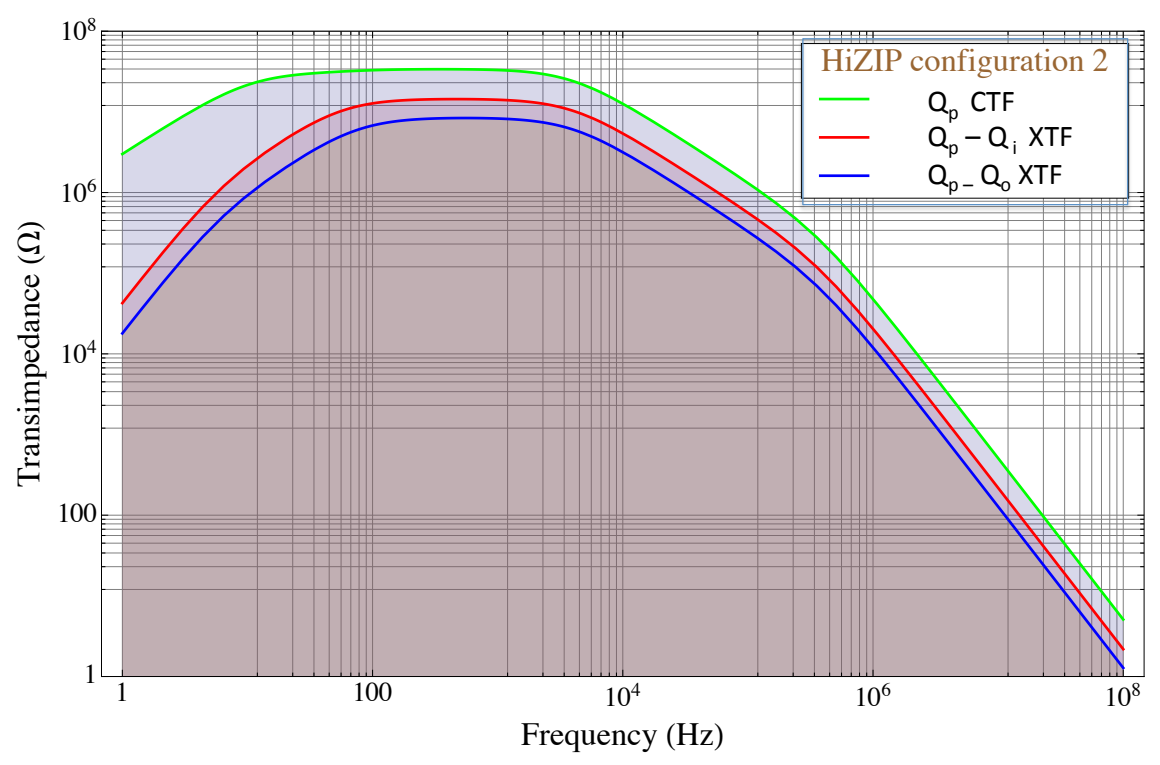

Figure 5.10: $\mathrm{Q}_{p}$ channel and crosstalk transfer functions for HiZIP configuration 2 .

\subsubsection{Electronic noise estimation in charge readout circuit}

To make a precise measurement of ionization as well as to be able to detect low energy interactions, we need to have as little noise as possible in our readout circuits. Johnson noise from the bias and feedback resistors and the voltage noise of the FET in the op-amp are the main sources of electronic noise in the ionization readout circuit. The FET noise is modelled as a voltage noise source with a spectral density of 0.5 $\mathrm{nV} / \sqrt{\mathrm{Hz}}$ in series with input and feedback impedances at the gate of the op-amp [70]. The Johnson noise of the bias and feedback resistors are modelled as current noise sources in parallel to the resistors. The RMS value of Johnson noise current of a resistor is given by:

$$
\mathrm{i}_{\mathrm{n}}=\sqrt{\frac{4 * \mathrm{k}_{\mathrm{b}} * \mathrm{~T}}{\mathrm{R}}}
$$


In order to find out the spectral density of all these noise sources at the output of the op-amp, we calculate the product of the voltage drop they cause at the gate of the op-amp with the voltage transfer function $\mathrm{H}(\mathrm{s})$. The total noise at the output is obtained by adding the contributions from each of the noise sources to the power spectral density at the output. We call this noise contribution the 'channel noise'. We calculate the noise contributions from coupled circuits ('crosstalk noise') in the same way. Figure 5.11 shows the spectral density of noise at the output of the opamp plotted versus frequency for a $Q_{i}$ channel in an iZIP detector. It also shows the contributions of noise from feedback and bias resistors and the FET noise. It can be seen that the FET noise dominates the total noise for frequencies higher than $~ 1$ KHz. Figure 5.12 shows the crosstalk noise contribution of the same components from the $\mathrm{Q}_{o}$ charge channel. Figure 5.13 compares the $\mathrm{Q}_{i}$ channel noise with the crosstalk noise from $\mathrm{Q}_{o}$. The crosstalk noise only contributes at the percent level to the total noise. For details of the calculation refer to appendix $\mathrm{A}$.

Similarly we can calculate the noise contributions to the new charge channel in both the HiZIP configurations. Figure 5.14 and 5.15 show the channel noise and crosstalk noise contributions to the total noise in the $Q_{p}$ channel for HiZIP configurations 1 and 2 respectively. The crosstalk noise contribution is much higher in the case of the HiZIP, although not high enough to affect our measurements adversely. 


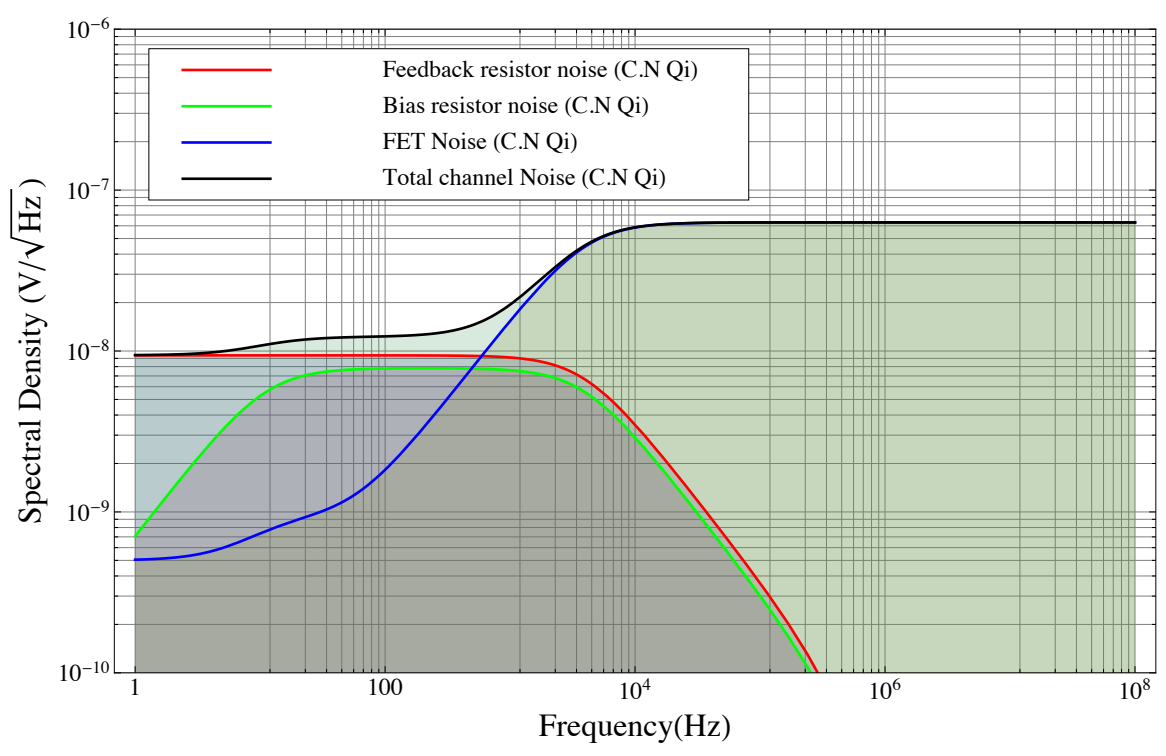

Figure 5.11: Noise at the output of $\mathrm{Q}_{i}$ from noise sources within $\mathrm{Q}_{i}$ readout circuit alone (C.N stands for channel noise). The individual noise contributions are added in quadrature to obtain the total noise at the output.

Figure 5.16 shows a comparison of the total noise in an oZIP (original ZIP with 4 phonon sensors on one side and 2 charge electrodes on the other used in CDMS II) , iZIP and the two configurations of HiZIP detector. It can be seen that the HiZIP total noise power in $\mathrm{Q}_{p}$ in either configuration is not considerably different from that of the iZIP $Q_{i}$. Although the total noise is calculated for $Q_{p}$ alone and not $Q_{i}$ for the HiZIP configuration 2, it is expected to be similar if not less. The noise calculation for the oZIP detector $\mathrm{Q}_{i}$ channel assumes a sensor capacitance to ground of $\mathrm{C}_{d}=50$ $\mathrm{pF}\lceil 70]$. 


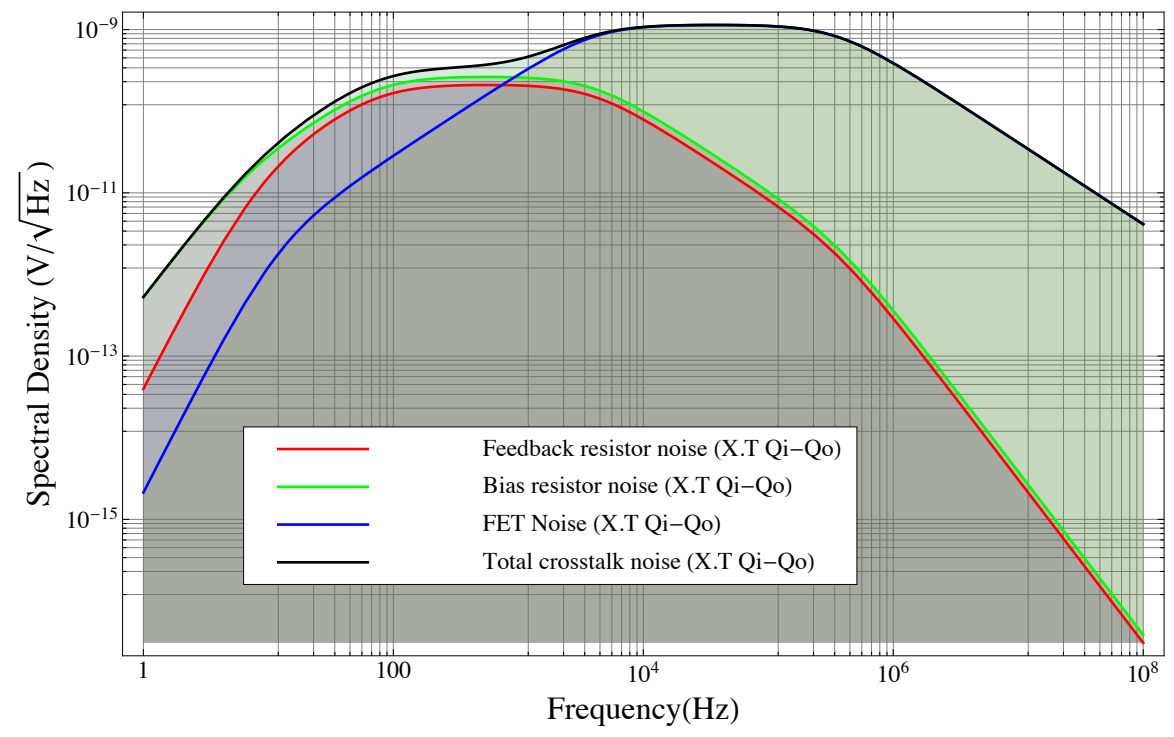

Figure 5.12: Crosstalk noise at the output of $Q_{i}$ from noise sources within $Q_{o}$ readout circuit alone (X.T stands for crosstalk).

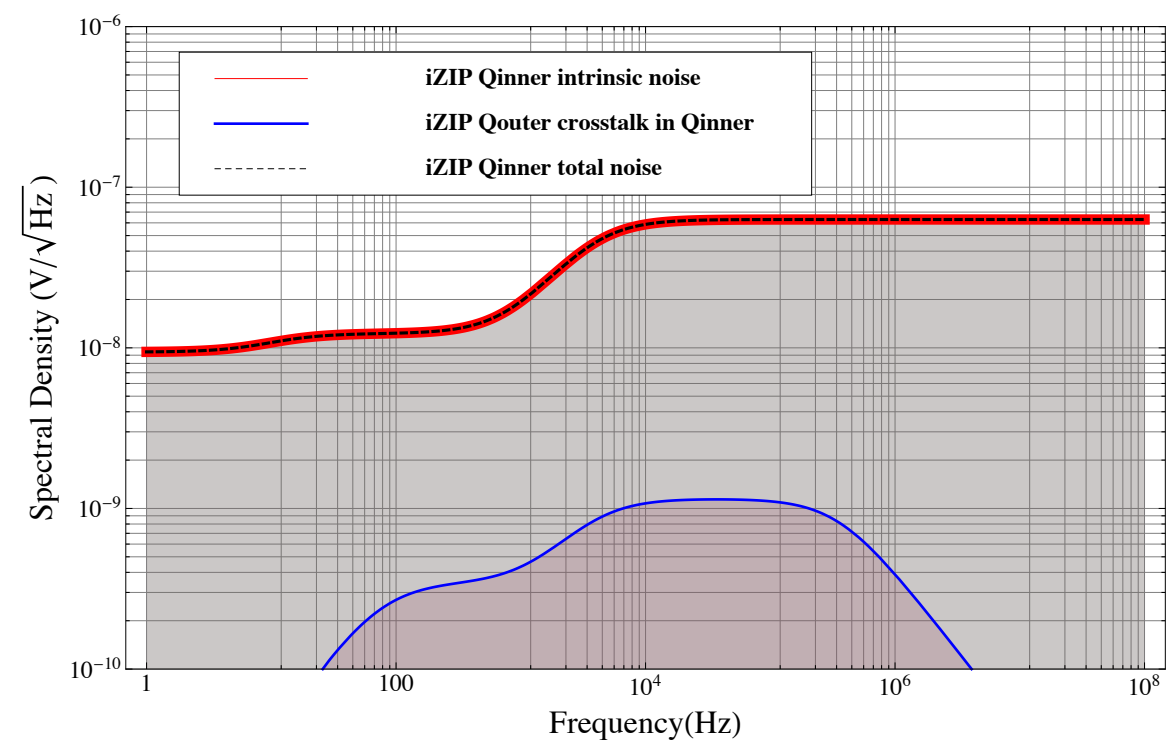

Figure 5.13: Total noise at the output of $Q_{i}$ (i.e. $Q_{i}$ channel noise $+Q_{i}-Q_{o}$ crosstalk). 


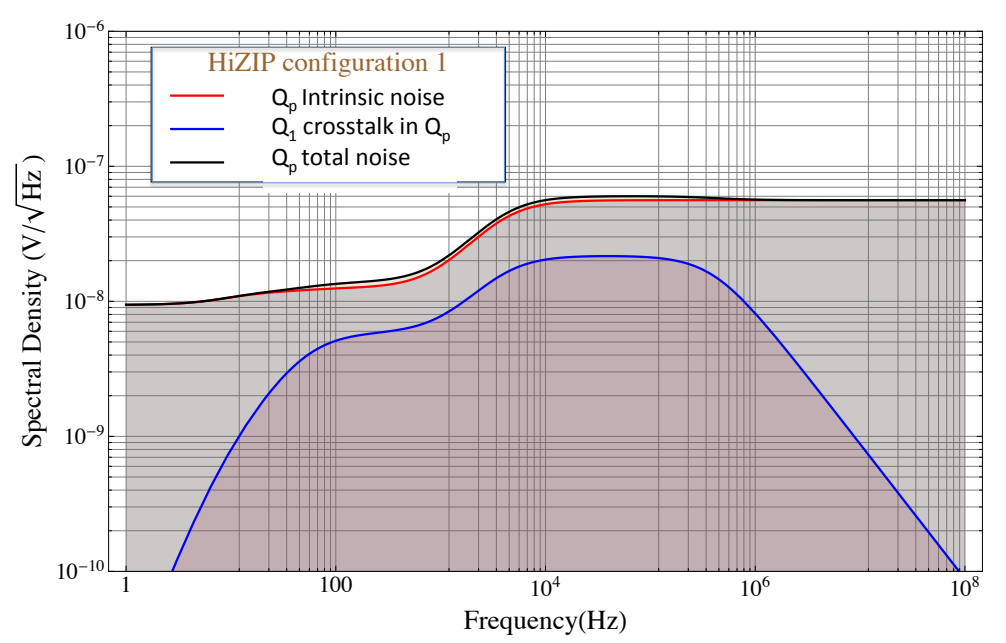

Figure 5.14: Noise at the output of $Q_{p}$ in HiZIP configuration 1. Note that $Q_{p}$ and $Q_{1}$ in this configuration have approximately equal capacitances and should indeed have identical noise spectrum at the output.

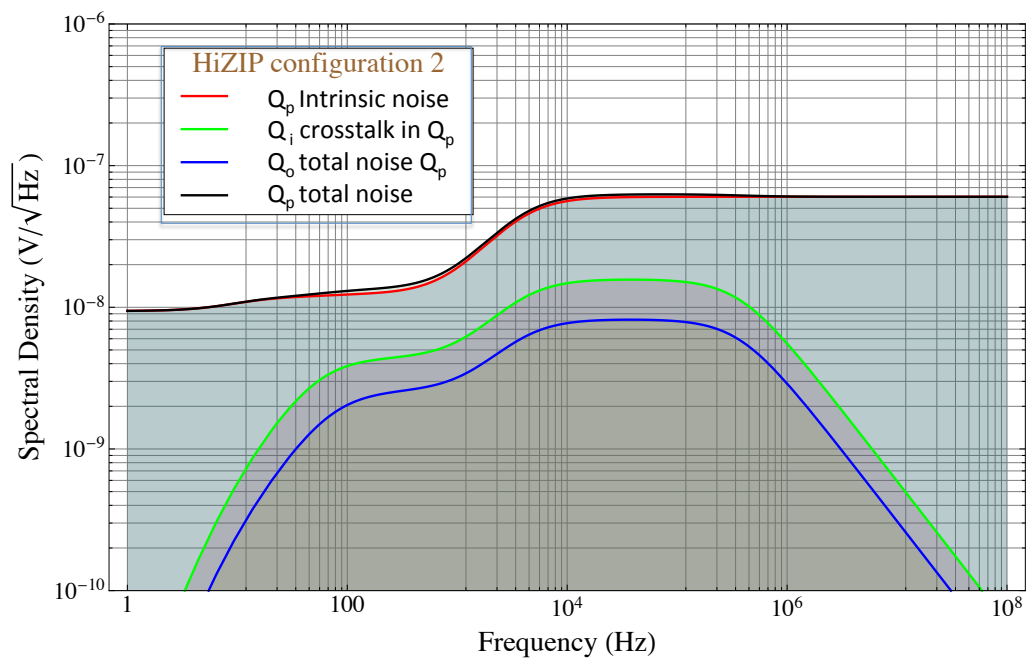

Figure 5.15: Noise at the output of $Q_{p}$ in HiZIP configuration 2. Crosstalk contributions from $Q_{i}$ and $Q_{o}$ are shown. 


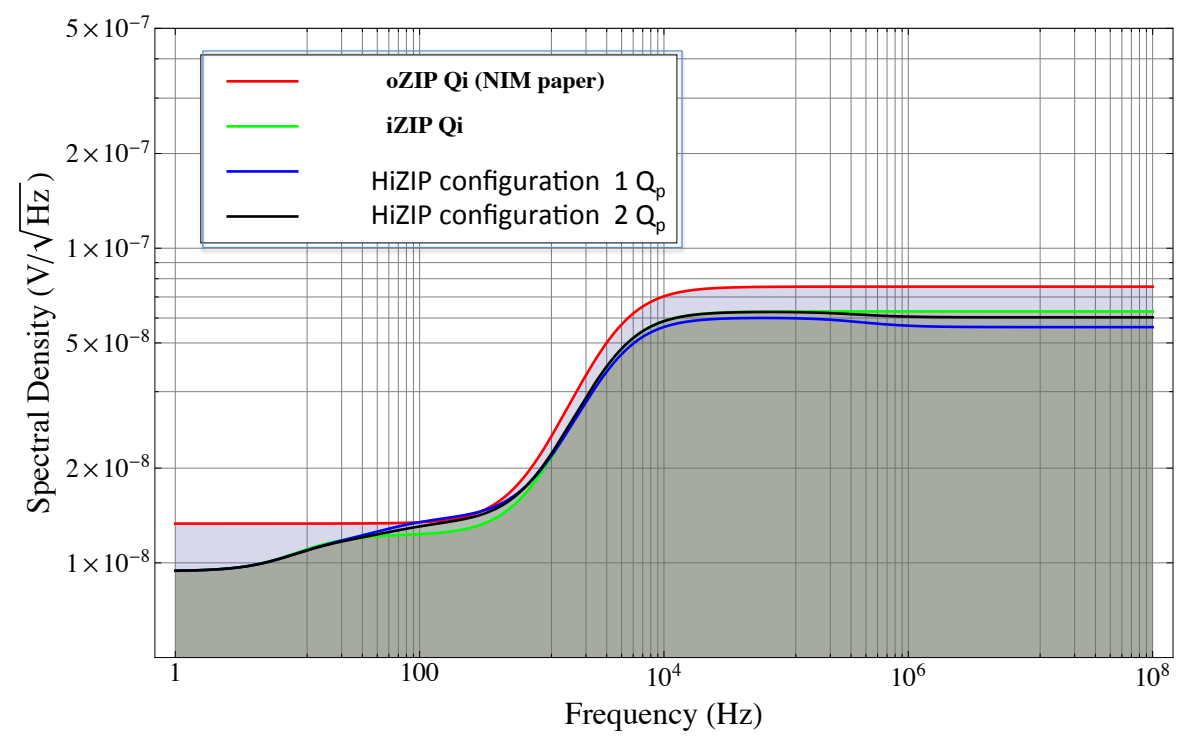

Figure 5.16: A comparison of the total noise at the output of the charge amplifier in different cases.

In conclusion, the effect of cross coupled noise is not significant enough to affect our measurements. However, a crosstalk correction like the one used for the iZIP analysis should eventually be implemented for an improved energy estimation.

\subsection{HiZIP viability studies using G48 iZIP data.}

The availability of iZIP data meant that we could gauge the performance of the HiZIP before we built it. This, however, is only true to an extent since the HiZIP not only allows us to discriminate against MISS events, but also provides an extra handle against surface events on one side. We used a subset of information from the iZIP data to reflect the data that would come out of a HiZIP (except the new interleaved charge channel). In the HiZIP, we have phonon sensors on a single side, and we have 
to create robust phonon energy estimators based on one side information only. While we gain a new charge channel, we also loose the ability to discriminate against $Q_{i n n e r}$ and $Q_{\text {outer }}$ events on one side. In summary we can construct a 'HiZIP dataset' using the following from iZIP data:

- Phonon information from one side only.

- Explicit $\mathrm{Q}_{\text {inner }}$ and $\mathrm{Q}_{\text {outer }}$ from one side only, while adding the $\mathrm{Q}_{\text {inner }}$ and $\mathrm{Q}_{\text {outer }}$ energies on the other side to mimic the data from a single large charge sensor.

We choose different quantities from the iZIP data that reflect the different hardware and bias configurations of the HiZIP. These different configurations are a combination of the 'side of the phonon sensors', definition of fiducial volume (effective detection volume of a detector) and the bias polarity. These represent the different data-taking modes of all the possible HiZIP hardware configurations. Using the data taken with the G48 iZIP detector at the UC Berkley's SuperCDMS test facility we carried out studies to estimate the fiducial volume and the surface event discrimination ability of the HiZIP detector for all possible configurations. These studies form the bulk of chapter 6 .

\subsection{The G16K HiZIP.}

Meanwhile an iZIP detector was re-wired to a HiZIP configuration (G16K). The G16K detector had its charge sensors wired in the HiZIP configuration 2 discussed in section 5.1. A couple points should be noted before we discuss about the G16K HiZIP:

- The charge channel on side 2 was obtained by physically combining $Q_{i n n e r}$ and Qouter sensors is referred to as $Q_{2}$. 
- $Q_{1}$ will refer to an analogous combination of $\mathrm{Q}_{\text {inner }}$ and $\mathrm{Q}_{\text {outer }}$ ionization energies on side 1 rather than a physical combination.

Figure 5.17 shows the G16K HiZIP with the charge sensors named in this convention.

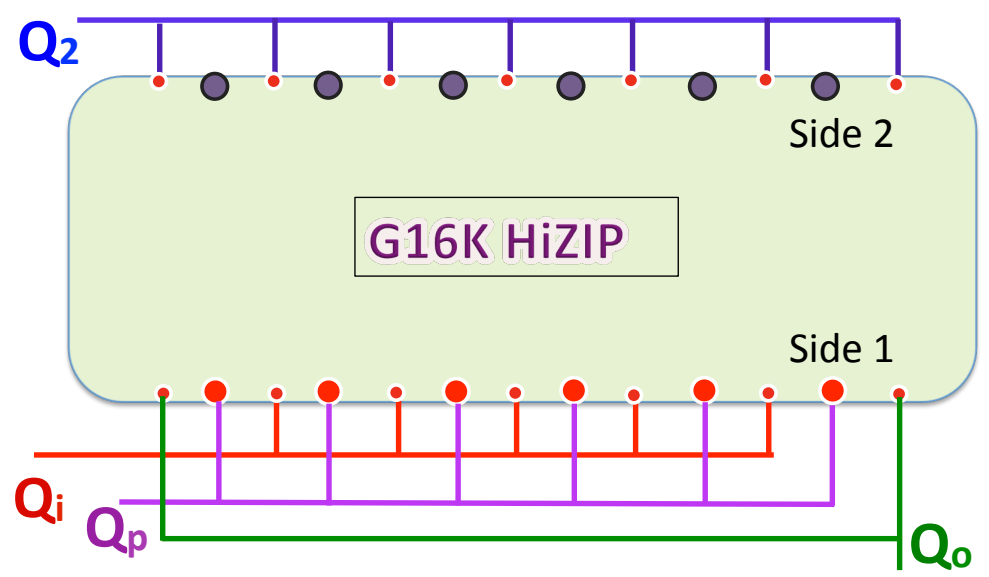

Figure 5.17: The G16K HiZIP charge sensor wiring scheme in the G16K naming convention.

Studies to estimate the fiducial volume and surface event discrimination of G16K were done using data taken at the SuperCDMS Queen's test facility. Chapter 7 describes these studies in detail. For basic studies $Q_{p}$ is not needed and therefore not discussed in chapter 7. However, here we want to demonstrate that $Q_{p}$ performs roughly as expected. Figure 5.18 shows a plot of ionization energy on $Q_{2}$ versus ionization energy on $Q_{1}$ coloured by the ratio of ionization energies on $Q_{p}$ and $Q_{1}$. The surface events on side 2 and 1 should lie along the vertical and horizontal axes respectively, while the bulk events which have a signal on both $Q_{1}$ and $Q_{2}$ should form a band along the line of slope one. Notice the events lying along the $Q_{1}$ axis have a 
$Q_{p}$ signal that is roughly proportional to $Q_{1}$ signal. Also the bulk events which are along the diagonal band have no visible signal on $Q_{p}$ as we expected. Then, there are events which are not perfectly bulk or perfectly surface events which have a smaller $Q_{p} / Q_{1}$ ratio and are spread between the side 1 surface events band and the diagonal bulk events band.

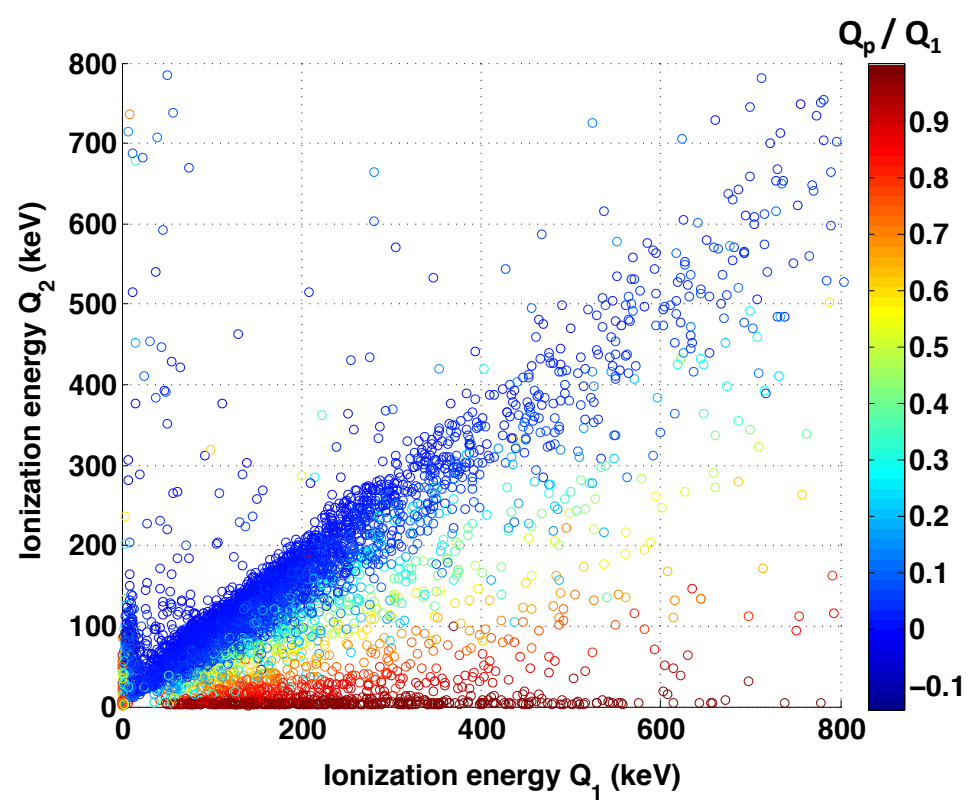

Figure 5.18: Ionization energy on $Q_{1}$ vs. $Q_{2}$ coloured on a linear scale by the ratio of $Q_{p} / Q_{1}$. We can see that only events close to side 1 surface (close to horizontal axis) produce a signal on $Q_{p}$ that is roughly proportional to $Q_{1}$.

Figure 5.19 shows the uncalibrated ionization energy on $Q_{p}$ versus the ionization energy on $Q_{1}$ coloured by $Q_{2}$. Notice that there are two bands: one where $Q_{p}$ is proportional to $Q_{1}$ and the other where $Q_{p}$ is very small and almost independent of $Q_{1}$. The former are surface events on side 1 , while the latter are a mix of surface 
events on side 2 and bulk events.

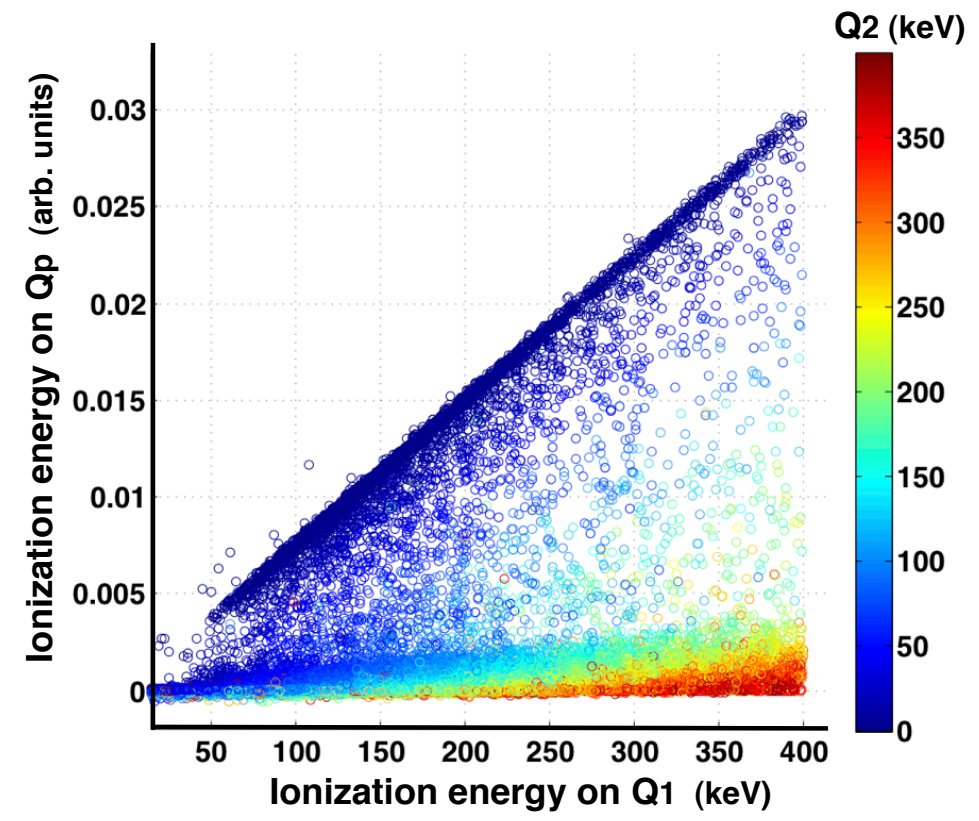

Figure 5.19: A plot of $Q_{p}$ vs. $Q_{1}$ coloured by $Q_{2}$ shows that side 1 surface events produce proportional signals in $Q_{1}$ and $Q_{p}$.

In summary, the new charge channel $Q_{p}$ carries useful information and behaves as expected as far as these few simple tests can show. However, there is scope for further work in realizing the potential of $Q_{p}$. 


\section{Chapter 6}

\section{G48 HiZIP Analysis}

As mentioned in the previous chapter, the viability of an iZIP like detector with phonon sensors on one side only was first evaluated using the data taken with the G48 detector at the SuperCDMS test facility at UC Berkley. In all the data sets used in this study, there was a ${ }^{109} \mathrm{Cd}$ beta source present on one side of the detector. Data taken with a ${ }^{133} \mathrm{Ba}$ source are used for characterization of the electron recoil response and energy calibration while data taken with a ${ }^{252} \mathrm{Cf}$ source are used for characterization of the nuclear recoil response. G48 is an iZIP detector and has 4 phonon sensors and 2 charge sensors on each side as explained in chapter 2 . However for this study phonon information from only one side was used. This detector had been studied extensively and a considerable amount of analysis had already been done on the data to be used, so the performance could be compared to a full iZIP analysis. However, the surface event source $\left({ }^{109} \mathrm{Cd}\right)$ is mounted on one side only for all the data used in this study. Hence, we study both polarities with the intent of studying surface events on the positive or negative side. As a first step, phonon energy estimators based on phonon information from a single side were constructed. 
The following section describes the first phase of the analysis that was carried out by other members of the SuperCDMS group at Queen's. It compares the performance of phonon energy estimators based on a regular optimal filter (OF) and a non stationary optimum filter (NSOF) using phonon information from a single side. Sections 6.2 onwards discuss the data analysis carried out using true HiZIP quantities, the phase of the G48 HiZIP project that I was most involved with.

\subsection{Analysis using sum based optimal-filter quan- tities}

As discussed in chapter 2, the optimal filter algorithm fits an averaged pulse template to the trace in the frequency domain while accounting for the noise. In this study, two phonon energy estimators were created at first. One based on the sum of the reconstructed OF phonon energies by fitting individual phonon traces from a single side of the detector called psum\# quantities (\#=1 or 2 indicates side the detector) and the other is the reconstructed OF energy for a fit of the summed phonon trace of sensors from a single side called ps\# quantities. The psum\# quantities were found to provide a better energy resolution and a tighter yield band. However there was considerable position dependence in the estimation of the phonon energy, specifically events happening close to the phonon sensors would have an overestimation of the phonon energy compared to those happening in the bulk of the detector. This is because events happening close to the surface of the detector tend to have peaky pulses (tend to have a sharp spike at the beginning of the pulse) and the optimal filtering algorithm in use tends to de-weight low frequencies in the fitting 81] [82. However 
most of the position independent information lies in the long tail (low frequencies) of the phonon trace coming from phonons absorbed uniformly across all sensors [83].

The phonon energy estimators calculated using 'non-stationary optimum filter' (NSOF) algorithm have proven to have much less position dependence. The nonstationary optimal filter treats the position dependent peaky part of the pulse (roughly within $100 \mu$ s from the start of the pulse) as non stationary noise and de-weights it in the frequency domain fit of the trace [82], thus reducing the position dependence in energy estimation. The plot of yield vs. recoil energy for a iZIP detector using NSOF based phonon energy estimator is shown in figure 6.1. The black band of events are mostly surface events from the ${ }^{1} 09 \mathrm{Cd}$ source source placed close to side 1 surface. Solid blue lines indicate the 'gamma band' (bulk electron recoil, also called ER band), solid green lines demarcate the 'neutron band' (bulk nuclear recoil, also called NR band) and solid red lines demarcate the region $3 \sigma$ below the gamma band and $3 \sigma$ above the neutron band, usually referred to as the 'beta band'. 


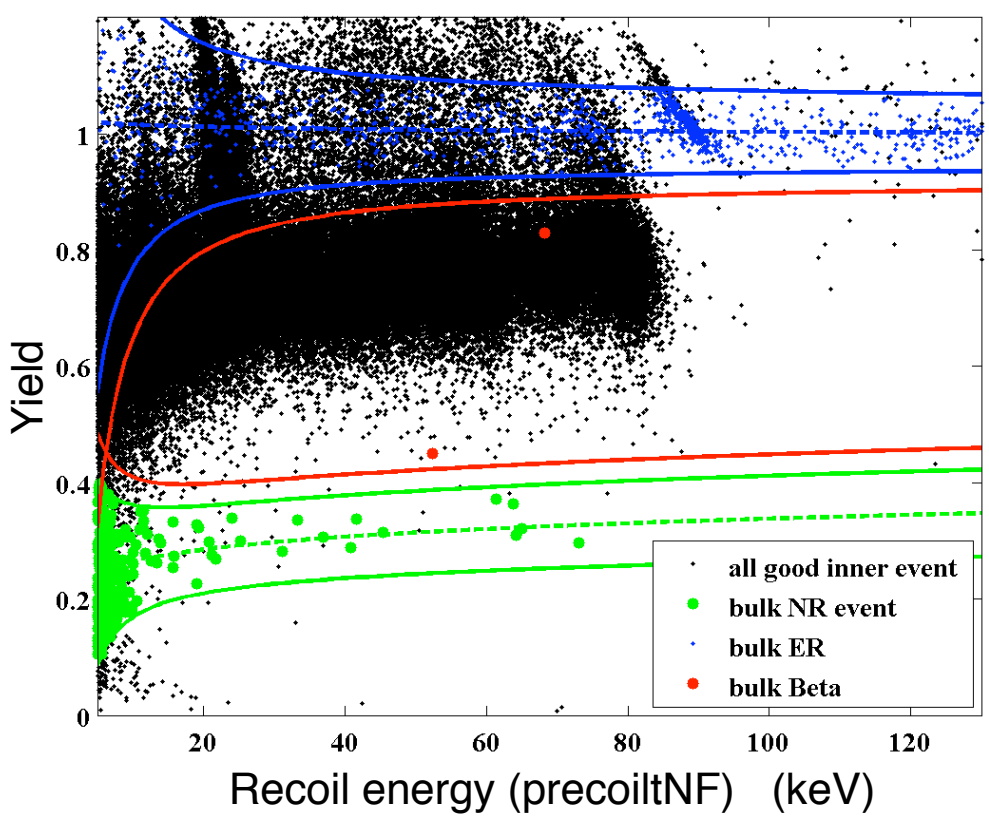

Figure 6.1: Yield vs. recoil energy for dataset taken with ${ }^{1} 09 \mathrm{Cd}$ source on side 1 . The line like structures are from gammas of specific energies from the source. Figure from [84])

In the subsequent phase of this study the same data was reprocessed with the NSOF algorithm to create new side based HiZIP phonon energy estimators called psNF\# quantities. Figures 6.2 and 6.3 show plots of yield vs. recoil energy, using OF and NSOF based estimators, for the same data sample as shown in figure 6.1. prec\#_hiZ and prNF\#_hiZ are the recoil energy estimates obtained from psum\# and psNF\# quantities after 'Luke correction'.

In the left plot of figure 6.2 the black band of events (mostly surface electron recoils on side 1 from ${ }^{109} \mathrm{Cd}$ betas) have lower yield compared to electron recoils in the bulk (indicated by the ER band in blue) and are encroaching into the NR band. This is because prec1_hiZ overestimates the phonon energy for events happening close 
to the side 1 surface while prec2 hiZ underestimates the phonon energy and the band ends up with a much higher yield and a much smaller recoil energy end point. The prNF\# hiZ quantities also show position dependence, albeit much less than the OF based quantities. More importantly, in the case where the phonon sensors are on side 2, although the effect is quite drastic, the yield is overestimated and hence these events do not pose the threat of leaking into the signal region. With this study the NSOF based quantities emerged as the phonon energy estimator of choice for the HiZIP.

The selection or exclusion a group of events made in the analysis is referred to as a 'cut'. Cuts in analysis are made for several reasons including selection of good quality data, defining the fiducial volume (effective detection volume) of the detector etc.. Up to this point all the analysis was done using data quality cuts (to select good data) which used phonon information from both sides. In the next phase we made data quality cuts (referred from here on as "quality cuts") using only single side phonon information and re-did the same analysis on the new data selection. If we can demonstrate comparable performance to an iZIP with this analysis, we can confidently assert the HiZIP's effectiveness as a dark matter detector. The following section will describe in more detail different stages of this 're-analysis'. 

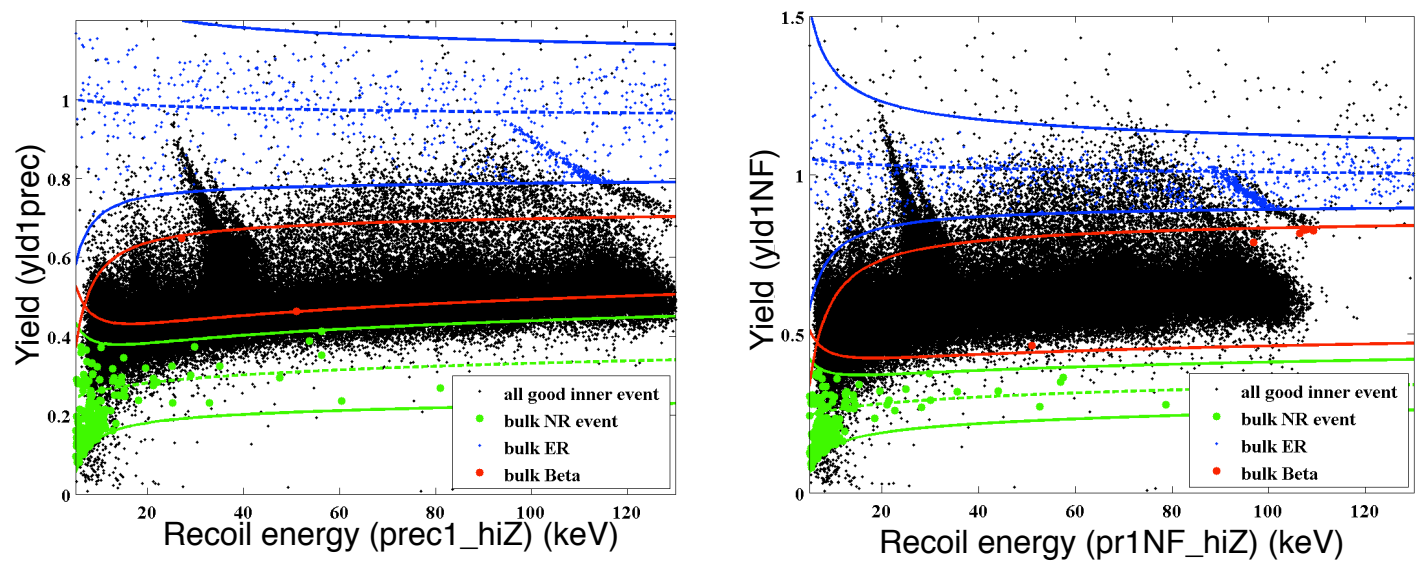

Figure 6.2: Yield vs. recoil energy using psum (OF) and psNF (NSOF) quantities for phonon sensors on 'Side 1'. Figure from 84].
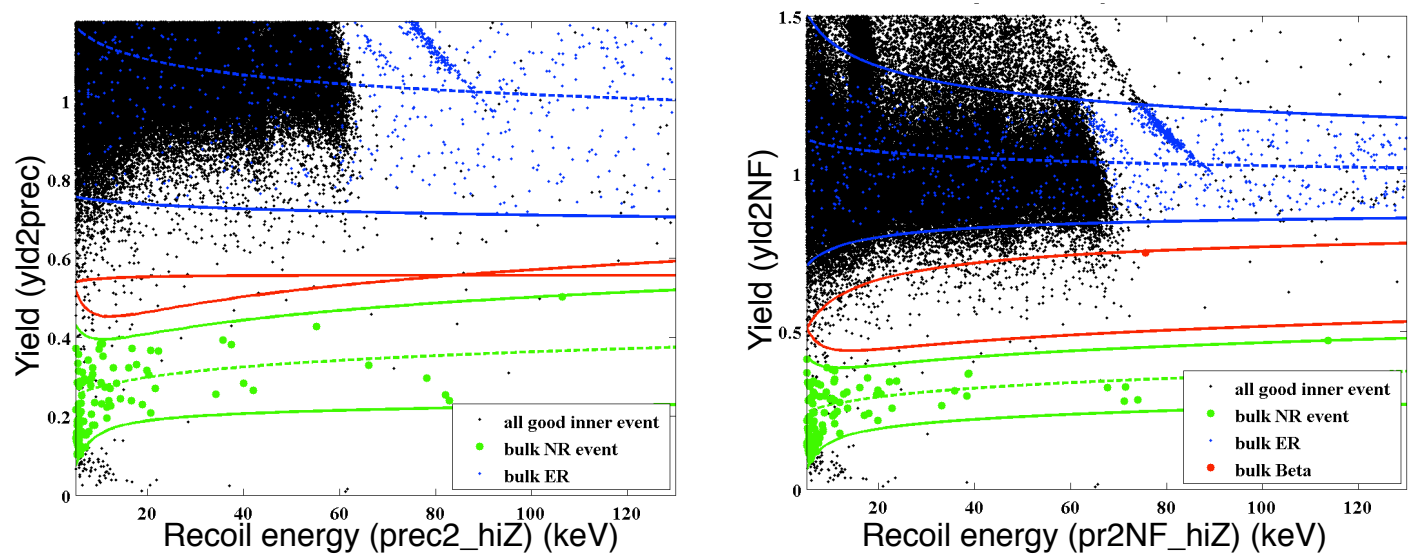

Figure 6.3: Yield vs. recoil energy using psum (OF) and psNF (NSOF) quantities for phonon sensors on 'Side 2'. Figure from [84]. 


\subsection{Full G48 HiZIP analysis with HiZIP data qual- ity selection.}

As discussed earlier, there are three main differences between a HiZIP detector and regular iZIP detectors. Firstly it has phonon sensors only on one side (top or bottom) of the detector, secondly the ground electrode on the 'no-phonon-sensor' side is instrumented with a read-out for ionization signal, and thirdly given the constraints from the current SuperCDMS cold hardware setup we end up losing Qinner-Q $_{\text {outer }}$ discrimination on one side by combining the inner and outer charge channels to accommodate the new charge channel. This means that we not only have to re-develop phonon based data quality selection using single side information but also a single sided charge based radial cut for the definition of the fiducial volume.

In order to do a thorough analysis we have to consider all the possible combinations in terms of hardware and biasing for the chosen design. This includes the side for phonon sensors, the side with $Q_{\text {inner }}$ and $Q_{\text {outer }}$ channels and the polarity of charge bias. The same data that was used in the previous studies comparing OF and NSOF based phonon estimators is used here. After defining the data quality cuts and gamma and neutron bands we calculate the efficiencies of the fiducial volume cut in the electron recoil band and in the nuclear recoil band (signal region) and make a comparison to the iZIP. In the end we make estimates for the leakage of surface betas into the signal region and compare it with those from the iZIP. By doing the analysis for iZIP and HiZIP designs on the same data we make the most direct comparison of the capabilities of the HiZIP and iZIP detectors. 


\subsubsection{HiZIP data quality cuts and fiducial volume cuts}

The charge based quality cuts developed for the earlier iZIP analysis of the data under investigation here do not have any explicit dependence on the distribution of the signal between $\mathrm{Q}_{\text {inner }}$ and $\mathrm{Q}_{\text {outer }}$ and can therefore be used without modifications. However, the iZIP phonon based quality cuts were defined using information from all phonon sensors and thus have to be redefined in terms of side 1 and side 2 based HiZIP quantities.

\section{Phonon $\chi^{2}$ cut}

A cut on the phonon NSOF fit $\chi^{2}$ is very effective at removing a lot of bad pulses and is commonly used in CDMS data analysis. The cut is deduced by looking at traces of events in different regions of 'energy vs. $\chi^{2}$ ' space and finding a suitable curve in this space and cutting out all the events with a $\chi^{2}$ above this curve. We end up with an energy dependent $\chi^{2}$ cut as shown in figure 6.4. The functional form used in this case is a quadratic function of the uncalibrated phonon energy. 


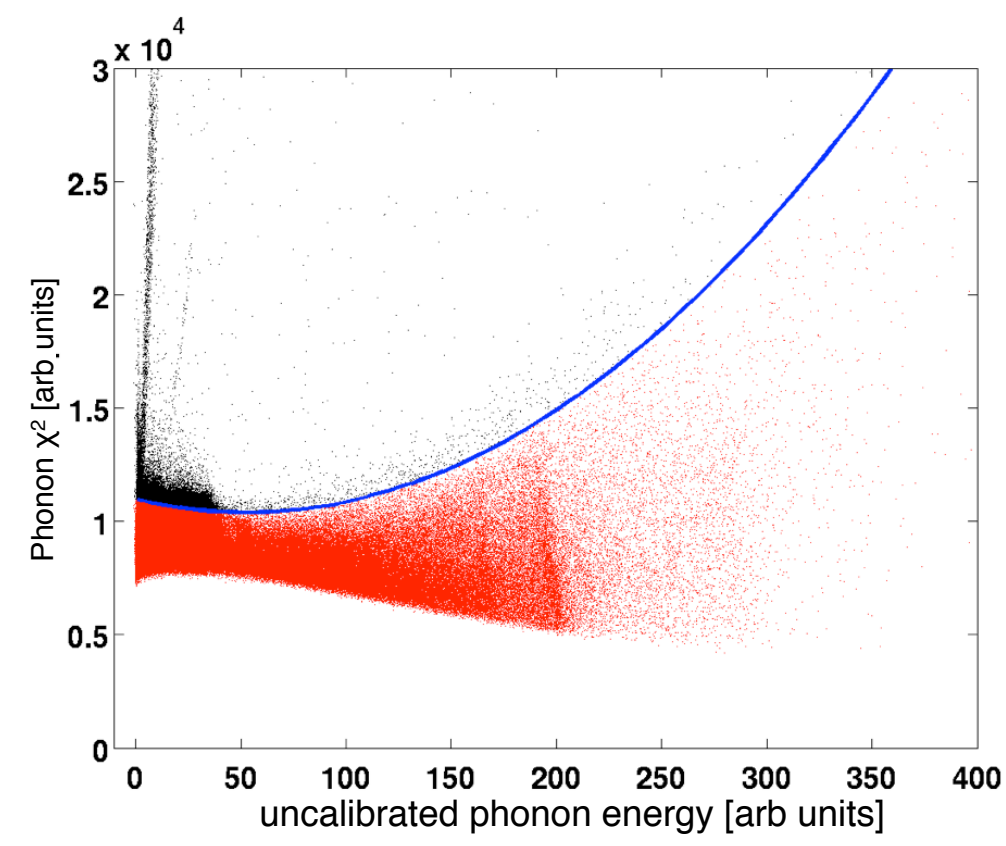

Figure 6.4: Example of phonon $\chi^{2}$ cut: phonon side $1 \chi^{2}$ vs. phonon energy (uncalibrated). Both the $\chi^{2}$ and phonon energy quantities are obtained from NSOF algorithm. The events below the curve, marked in red, are retained. Figure created by Chase Crewdson [85].

The cut is tighter at lower energies since for a given $\chi^{2}$ the energy estimation is relatively worse for a lower energy pulse compared to a higher energy pulse, in other words we can tolerate a higher $\chi^{2}$ at higher energies. The cut was implemented for NSOF based quantities of summed phonon traces from side 1 and 2 separately.

\section{Phonon pileup cut}

Pileup events are a class of events where two event pulses are not separated enough in time, in other words there is an overlap of pulses. These events could lead to poor 
fitting and incorrect energy estimation. Many of these events have poor fit $\chi^{2}$ and are removed by the $\chi^{2}$ cut. Despite the $\chi^{2}$ cut, many pileup events still remain and need to be removed explicitly from our data. There are two main categories of pileup events; one where more than one pulse is present in an event trace window and the other where a pulse sits on the tail of the previous pulse which is not seen in the given trace window (see figure 6.5).
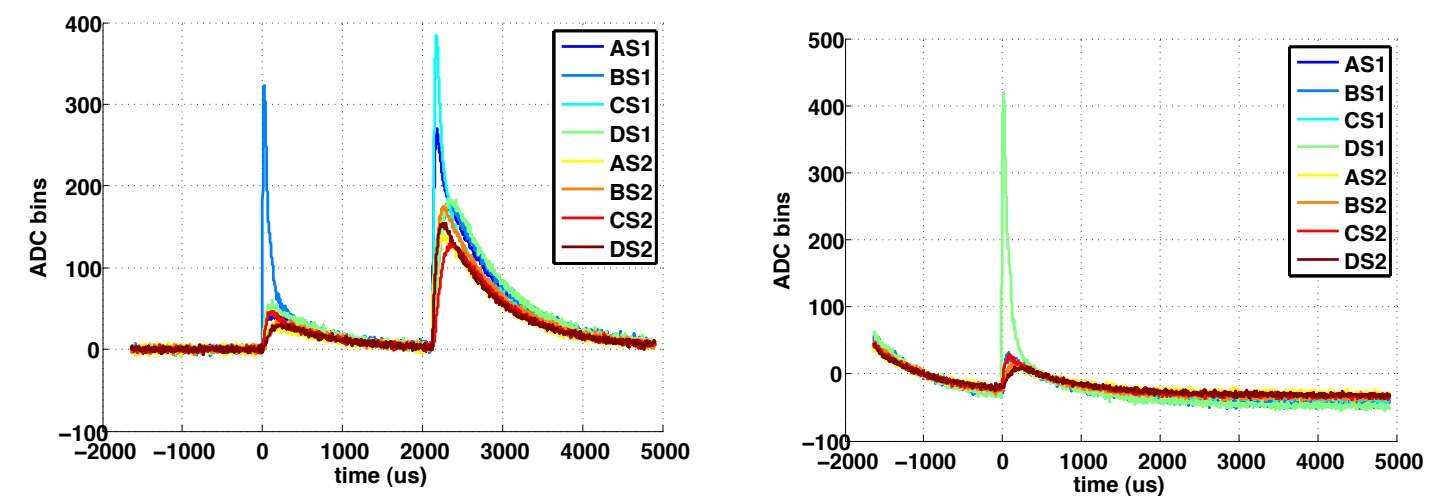

Figure 6.5: Examples of pileup events. The legend indicates colours used for the phonon channels A, B, C and D on side 1 (S1) and $2(\mathrm{~S} 2)$ respectively.

In order to remove these events a cut was placed on the ratio of the mean of the summed phonon trace (here on referred to as the pt trace) over the last 100 bins to the total phonon energy estimated from NSOF algorithm. This cut was implemented in the analysis of the G48 as an iZIP detector, but we had to redefine the cut using HiZIP's side based quantities. As mentioned earlier the tail of the phonon pulse provides an unbiased estimate of the phonon energy and for a good pulse should be proportional to the NSOF energy estimate. However for a pileup pulse this ratio is expected to be skewed for either of the two classes of pileup events described. In the case where there are multiple pulses in the trace window the fitting algorithm makes 
a fit to the first pulse and hence the ratio is expected to be off due to the presence of the second pulse. In the case where the pulse sits on a slope, the fit itself is poor since the algorithm is not sensitive to the slope of the baseline, hence the ratio is skewed again.

As an example, a plot of the mean of last 100 bins of summed phonon trace of side 1 (PS1_end) versus the recoil energy (pr1NF_hiZ) is shown in figure 6.6. It can be seen that the ps1_end is proportional to the recoil energy. In order to simplify the cut we first define a new quantity, PS1end_fraction which is identical to PS1end but has this slope shown in the first plot of figure 6.6 subtracted. The pileup events lie away from the centre of this distribution and are cut out.
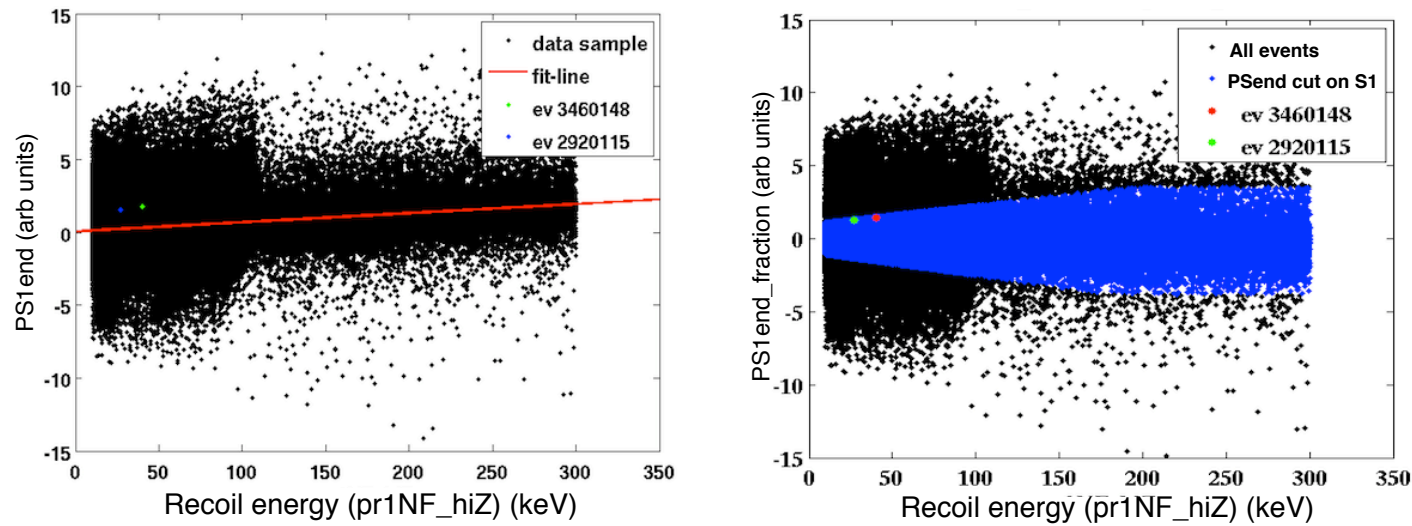

Figure 6.6: Left: Mean of the end phonon trace (last 100 time bins) vs. recoil energy. Right: Slope of distribution subtracted to simplify cut definition 

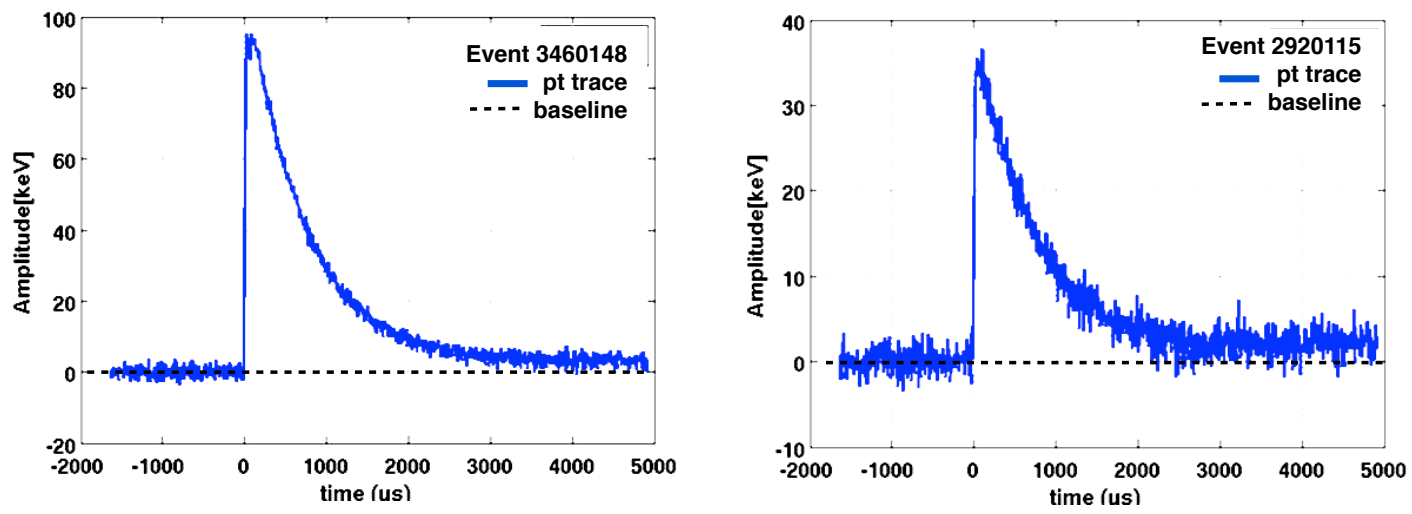

Figure 6.7: Summed phonon pulses (pt pulses) of events at the cut boundary. Left: Event Number 3460148. Right: Event Number 2920155 (for their position in the distribution refer to the highlighted events in figure 6.6 and the entry in the legend). These events do not show a clear pile-up feature but rather seem to sit on a slightly rising baseline which might be due to some low-frequency noise. The effect of this slope on the energy estimator is small; it is therefore justified to keep these events in the sample.

A region around the band mean is selected by looking at traces of events in different regions. The cut uses a pair of sloped lines at lower energies to make the selection tighter and a pair of horizontal lines at higher energies. The selection is marked in blue in the plot on the right plot of figure 6.6. A couple of events lying on the boundary are chosen and their pt trace is plotted in figure 6.7 to demonstrate that we end up with a clean data sample at lower energies. 


\section{A cut on phonon pre-pulse baseline standard deviation}

A cut on the phonon pre-pulse baseline standard deviation allows us to remove most of the sloped pulses (second class of pileups described above) and clean up the sample even further. Ideally the standard deviations are expected to have a gaussian distribution. However for sloped pulses the standard deviation will be higher. A $3 \sigma$ cut based on a Gaussian fit to the distribution of standard deviations removes most of the sloped pulses. Such a cut already existed for the iZIP analysis but had to be re-developed for the HiZIP phonon traces based on a single side.

\section{A cut to further remove any pileups events}

Another cut was made to remove events with an abnormally high derivative of the falling edge of the phonon pulse. This further removes pileup events from the sample.

\section{A cut to remove saturated phonon pulses}

There are events where the pulses are saturated or reading the minimum digitizer value due to the readout electronics going out of operational range. These events need to be removed. A quantity calculated on all phonon channels during data processing tells us the number of time-bins of the pulse that are above saturation or the number of bins that are at the digitizer minimum value. A data quality cut requiring that none of the channels on a given side are saturated was created for each side.

\section{A cut to remove 'Glitch events'}

In this data there were a significant number of events which were artifacts from the phonon readout circuit that had pulses of amplitude much higher than baseline noise 
but fall-times of about $200 \mu$ s which is considerably smaller than real phonon pulses which have fall times are around $750 \mu \mathrm{s}$.

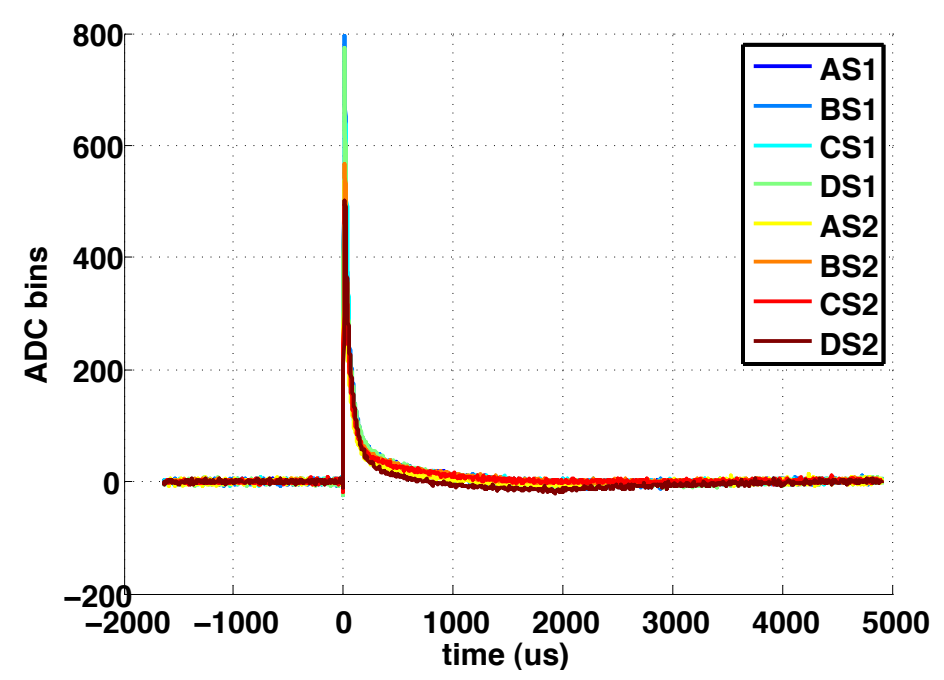

Figure 6.8: Phonon traces of a 'Glitch' event

Glitch events could pass the charge symmetry requirement at low energy and leak into the signal region due to low yield. The phonon traces of a glitch event are shown in figure 6.8. It can be seen that the fall-time is much smaller compared to real events (see figure 7.13). A cut was developed using the difference in fall times as shown in figure 6.9. The glitch events are easily identifiable in the lower part of the fall time vs.. rise time plane. Very long fall or rise times are indications for pulse pathologies as well therefore we also introduce a cut on the upper end of the distributions. 


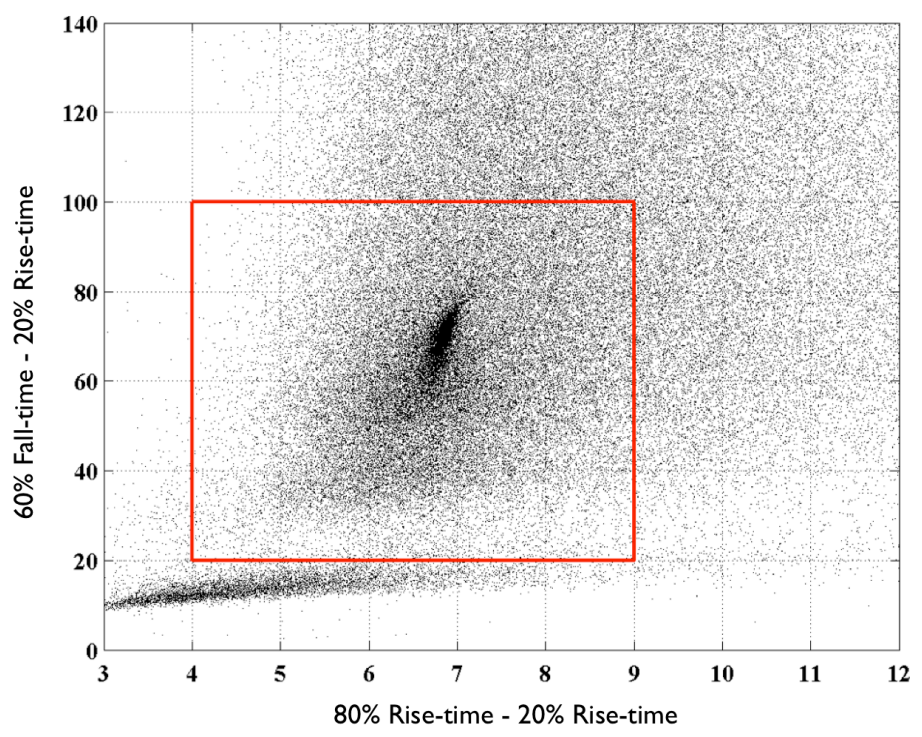

Figure 6.9: Glitch event cut created by Carlos Martinez [86].

\section{Charge symmetry cut for bulk event selection}

Bulk events are selected using the 'charge symmetry cut' which is placed in the $\mathrm{Q}_{\text {sum }}$ side 1 and $\mathrm{Q}_{\text {sum }}$ side 2 plane. $\mathrm{Q}_{\text {sum }}$ is the sum of energies in $\mathrm{Q}_{\text {inner }}$ and $\mathrm{Q}_{\text {outer }}$ channels on a given side (see figure 6.10). The cut selects bulk events which are distributed about the line of slope 1 . The cut was implemented for the iZIP analysis of the G48 detector by collaborators. This cut serves as a preliminary fiducial volume selection. It does not depend on any quantities not available in the HiZIP analysis and is therefore applied without changes. 


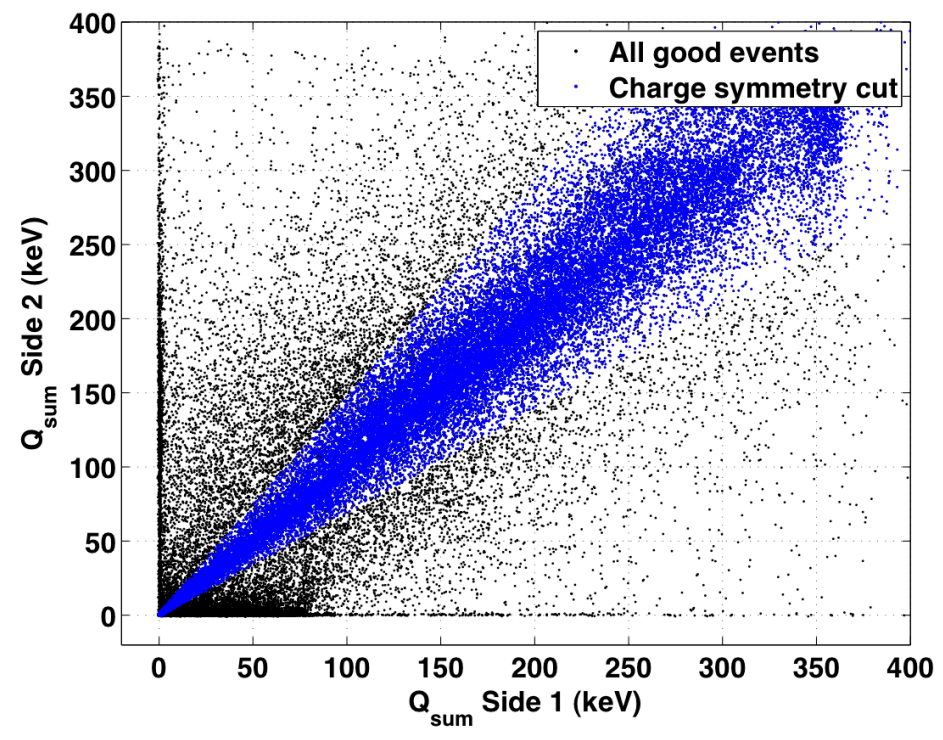

Figure 6.10: Charge symmetry cut for the G48 detector. The events passing charge symmetry cut are plotted over all good events which are selected by applying charge and phonon data quality cuts.

\section{Radial cut}

Regions closer to the cylindrical wall (also referred to as the sidewall) of the crystal do not result in full charge collection, hence we use the Qouter electrode as a guard ring and remove events close to this surface. The radial cut was chosen such that the fiducial volume remained constant at higher energies while being maximally inclusive at lower energies [87. The cut is placed in the $Q_{\text {inner }}-Q_{\text {outer }}$ plane as shown in figure 6.11 for side 1 . The cut was implemented for the iZIP analysis of the G48 detector by collaborators. We use only use one part of the cut for each of the configurations considered. The radial cut along with the charge symmetry cut define the fiducial volume based on charge information. 


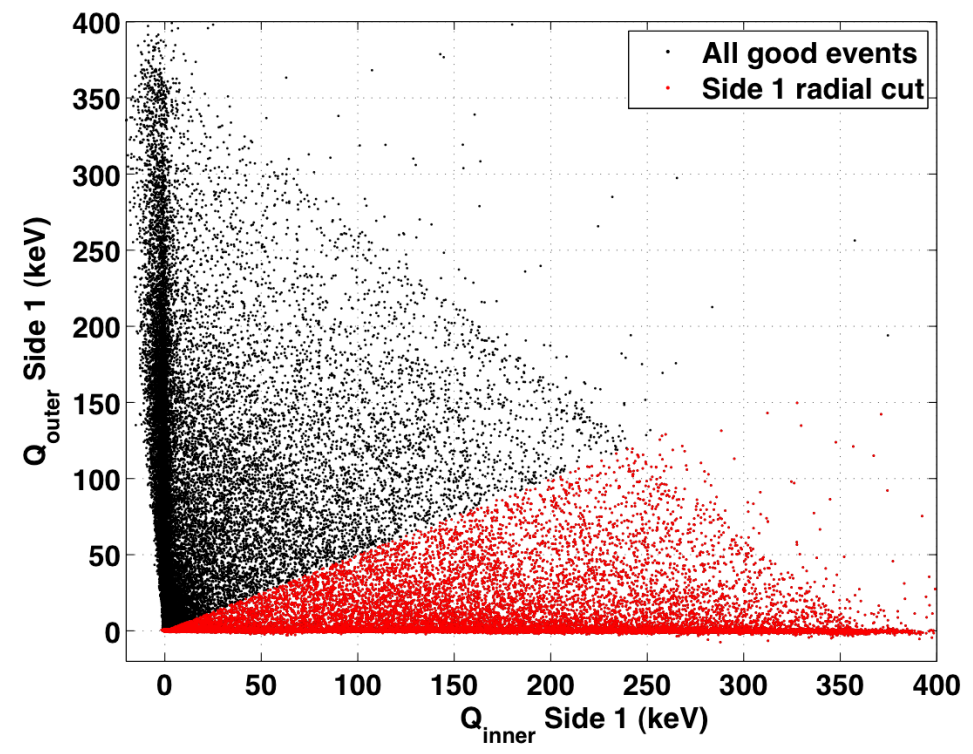

Figure 6.11: Side 1 radial cut for G48 detector. The events in red have a major portion of their signal in the $\mathrm{Q}_{\text {inner }}$ channel and are retained. The good events are selected by applying data quality cuts.

\subsubsection{Defining yield bands}

Once the good events within the fiducial volume are identified, yield bands are defined. The electron recoil (ER) band is defined using bulk gamma events. In this case a ${ }^{133} \mathrm{Ba}$ source was used to illuminate the detector. The yield for bulk electron recoils is normalized to one by setting the yield for the $356 \mathrm{keV}$ photo peak from the ${ }^{133} \mathrm{Ba}$ gammas in the bulk to one (see figure 6.12). The nuclear recoil (NR) band is defined using bulk nuclear recoils from neutron calibration runs with a ${ }^{252} \mathrm{Cf}$ source. Binned gaussian fits are made for both ER and NR bands, and functional forms fit to the bin-means. 


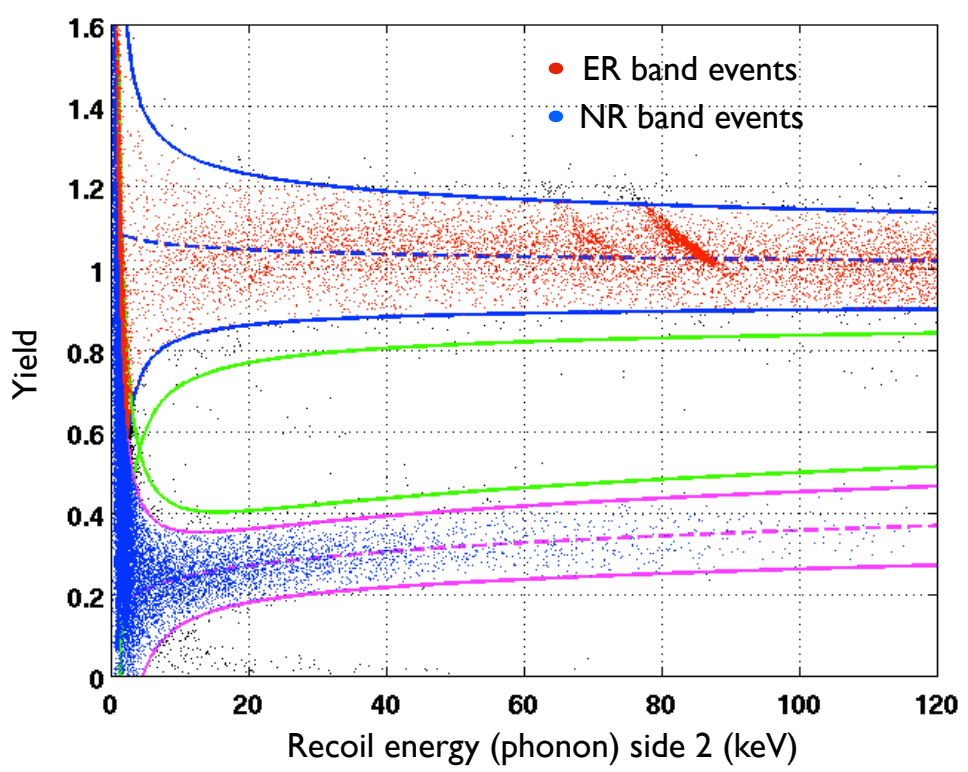

Figure 6.12: Yield vs. recoil energy for the HiZIP with phonon sensor on side 2. Solid blue and magenta curves mark the $2 \sigma$ bands for ER and NR respectively, while the green curves demarcate the beta band ( $3 \sigma$ below ER mean and $3 \sigma$ above NR mean). The data from ${ }^{133} \mathrm{Ba}$ and ${ }^{252} \mathrm{Cf}$ datasets used for ER and NR band definitions respectively are overlaid in this plot. Figure created by Chase Crewdson [88].

\subsubsection{Fiducial volume of iZIP vs. HiZIP}

The fiducial volume for the HiZIP detector is different from that of the iZIP. As mentioned earlier we define the fiducial volume based on charge information. For an iZIP detector, all events passing the radial cut on either side 1 or 2 and passing the charge symmetry cut are considered to be inside the fiducial volume. This allows us to efficiently remove events happening at high radius (close to side walls). However 
for the HiZIP we do not have the outer electrode on one side and hence will have to rely on the $\mathrm{Q}_{\text {inner }}-\mathrm{Q}_{\text {outer }}$ information from the other side alone. If we choose to select only $\mathrm{Q}_{\text {inner }}$ events from one side, we end up with the following two scenarios:

- If we are collecting electrons on this side we lose a considerable fraction of the fiducial volume as compared to iZIP since electrons propagate obliquely in our detectors [89 [90]. Events happening inside the fiducial volume would come from within a truncated cone such as shown in figure 6.13 (right).

- If we collect holes on this side we do not expect to lose very much in fiducial volume compared to iZIP since holes closely follow field lines and the fiducial volume is more cylindrical than conical. See figure 6.13 (left). 


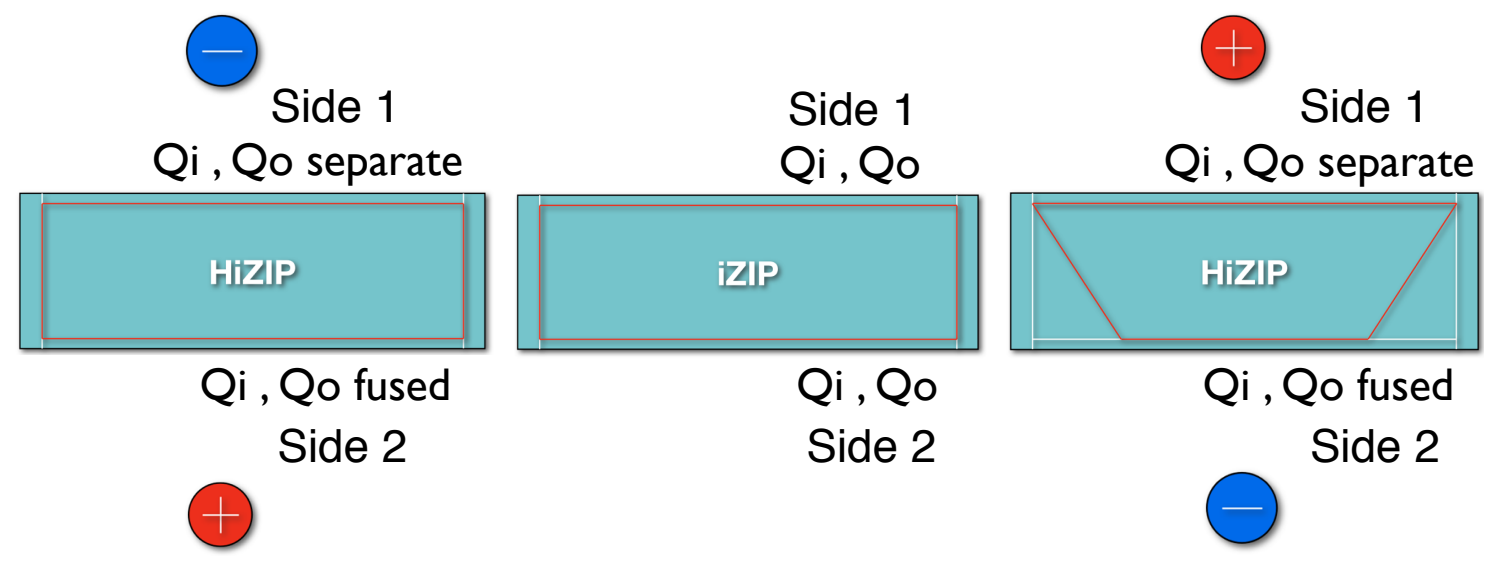

Figure 6.13: Fiducial volume of iZIP (middle) for either polarity defined by application of radial cut on either side. The fiducial volume loss due to oblique propagation of electrons in the HiZIP with side 1 positive bias (right) and a radial cut on side 1 . The fiducial volume remains roughly the same as an iZIP for the HiZIP side 1 negative bias (left) with a radial cut on side 1 since holes move along the field lines. Figure from [91].

It must be noted that the illustrations of the fiducial volume shown in figure 6.13 are geometrically inferred. Also, in light of studies showing charge build-up with time on the side walls of the detector, the true fiducial volume changes with time. The sidewall charge build-up, which seems to be predominantly electrons, makes the sidewalls more negatively charged and makes the hole signal move towards the outer electrode and electron signal move inward [92]. The efficiencies calculated below for the radial cut are time-independent and hence should be viewed in light of this effect.

Since the charge symmetry restriction can be applied identically in both iZIP and HiZIP the radial cut fully reflects the difference in the fiducial volume between iZIP and HiZIP. Hence it makes sense to calculate the efficiency of the radial cut alone 
after the application of the charge symmetry restriction. The efficiency of the radial cut is defined as the ratio of number of good events passing both the charge symmetry cut and the radial cut to the number of good events passing the charge symmetry cut alone.

Before we discuss the efficiency of the radial cut in different configurations, it is convenient to create some mnemonics for the cuts and quantities to simplify the discussion. Following are the names adopted along with their definitions:

- cQin1: Radial cut on side 1. Assumes Qinner and Qouter separation on side 1.

- cQin2: Radial cut on side 2. Assumes $Q_{\text {inner }}$ and Qouter separation on side 2.

- cQsym: Charge symmetry restriction; selects bulk events.

- cQin_or: Events passing radial cut on either side 1 or 2 . It is the maximally inclusive radial cut for an iZIP (assumes $Q_{i n n e r}$ and Q Quter separation on both sides).

- precoiltNF: Recoil energy for iZIP, based on phonon information from both sides of detector (Luke corrected). 'NF' here refers to the NSOF algorithm from which these quantities are obtained.

- pr1NF hiZ: Recoil energy for HiZIP, based on phonon information from side 1. Implies phonon sensors on side 1 alone (Luke corrected).

- pr2NF_hiZ: Recoil energy for HiZIP, based on phonon information from side 2. Implies phonon sensors on side 2 alone (Luke corrected). 


\section{Efficiency of the radial cut in signal region (NR band)}

The efficiency of the radial cut was calculated for events in the NR band for both bias polarities for different hardware configurations. The loss in fiducial volume in different energy bins is plotted in figure 6.14 for cQin1 as compared to cQin_or for both polarities. The same is plotted for cQin2 in figure 6.15.
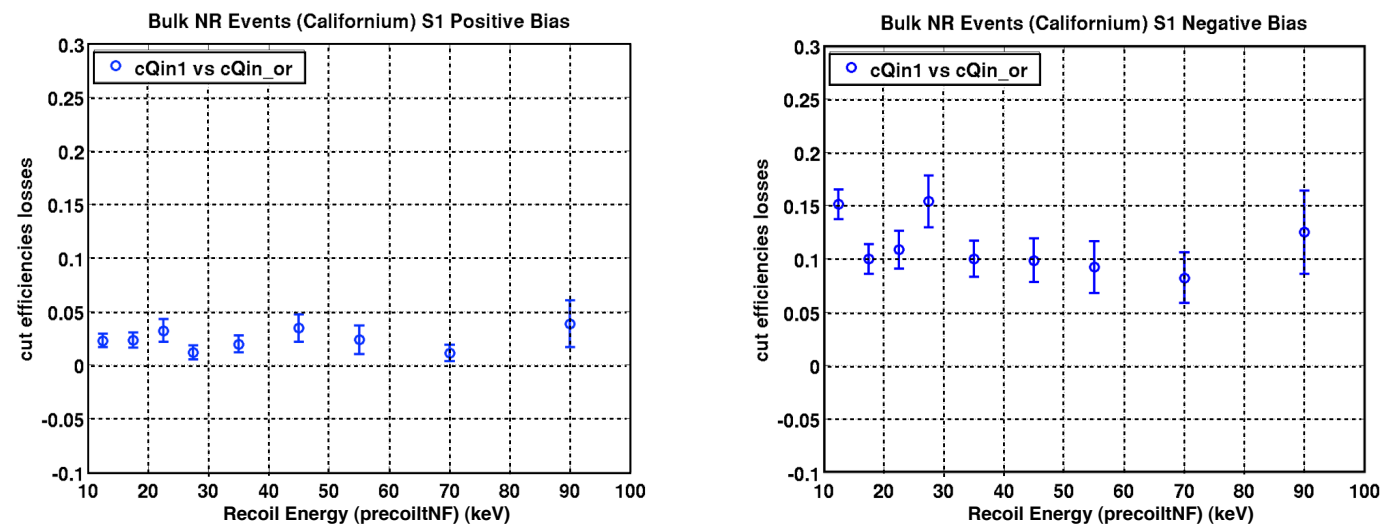

Figure 6.14: Efficiency losses (fractions) for cQin1 compared to cQin_or in different recoil energy bins in the nuclear recoil band for side 1 positive (left plot) and side 1 negative (right plot). The iZIP recoil energy (precoiltNF) is used since it is the best estimate of the recoil energy. 

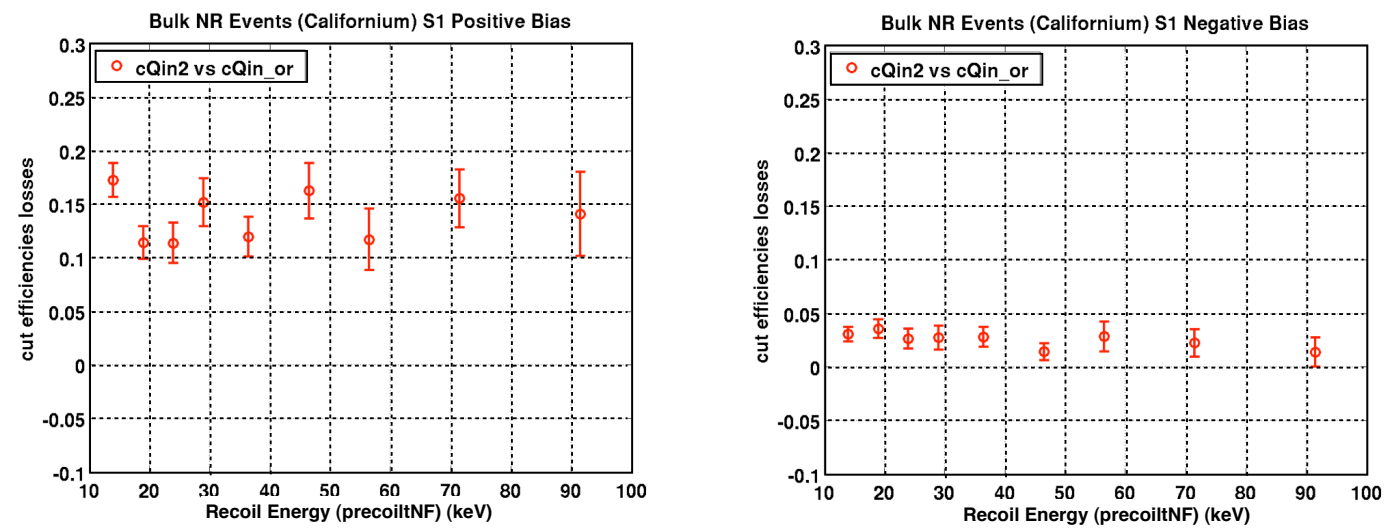

Figure 6.15: Efficiency losses for cQin2 compared to cQin_or in different recoil energy bins in the nuclear recoil band for side 1 positive (left plot) and side 1 negative (right plot).

We observe an energy averaged loss of fiducial volume of 3-4 \% for a HiZIP configuration compared to the iZIP for the case where the side with $Q_{\text {inner }} / \mathrm{Q}_{\text {outer }}$ separation has positive polarity bias and a loss of 10-15\% for the opposite polarity. This is contrary to our expectation about the fiducial volume loss discussed above based on purely geometrical considerations. This is likely to come from the sidewall charge build-up causing the electron signal to drift inward with time while the hole signal drifts outward. This translates to an increasing efficiency with time for electron collection and a decreasing efficiency with time for hole collection. It was noted that the trend was the same whether the recoil energy was constructed from phonon information from both sides or from any one side, implying it does not matter much whether the phonon sensor in on the side with $Q_{\text {inner }}$ and $Q_{\text {outer }}$ separation or not. This implies the data sample selected by applying data quality cuts based on side 1 and side 2 phonon estimators had most events in common and hence the estimation of fiducial 
volume is not affected very much by which side the phonon sensor is placed 88.

\section{Efficiency of radial cut in electron recoil (ER) band}

A similar trend for efficiencies was observed for events in the electron recoil band (see figure 6.16 and 6.17). Electron collection on the side with $\mathrm{Q}_{\text {inner }}$ and $\mathrm{Q}_{\text {outer }}$ separation resulted in better efficiency than hole collection.
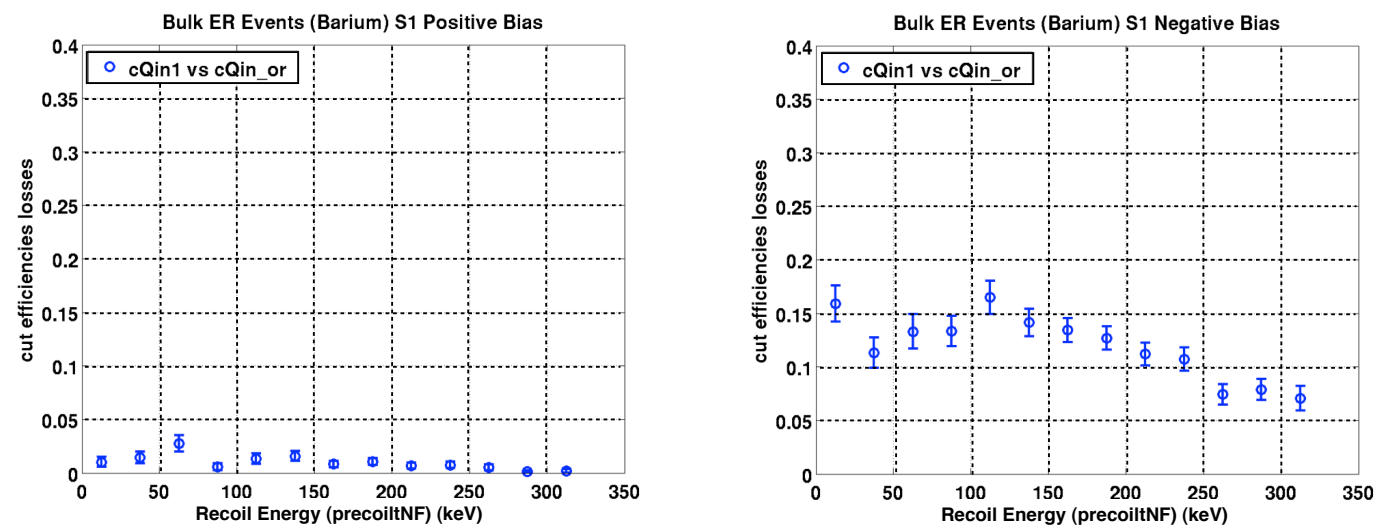

Figure 6.16: Efficiency losses for cQin1 compared to cQin_or in different recoil energy bins in the electron recoil band for side 1 positive (left plot) and side 1 negative (right plot). 

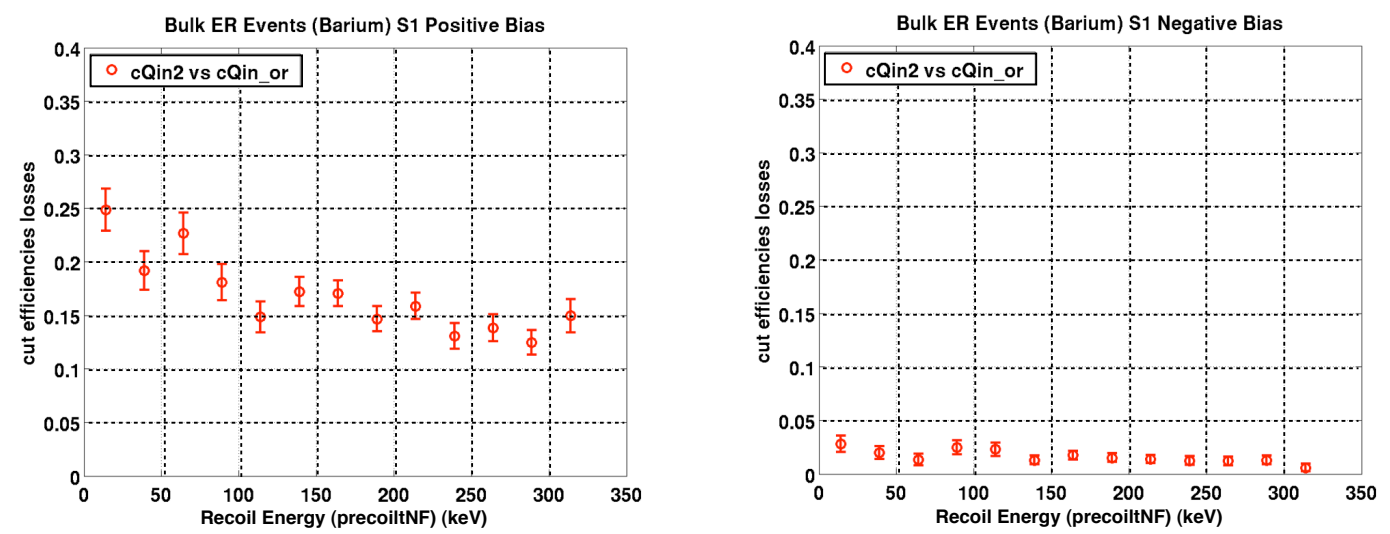

Figure 6.17: Efficiency losses for cQin2 compared to cQin_or in different recoil energy bins in the electron recoil band for side 1 positive (left plot) and side 1 negative (right plot).

\subsubsection{HiZIP surface event discrimination}

A surface event sample was selected by choosing the datasets which were taken with only the ${ }^{109} \mathrm{Cd}$ beta source (always on side 1 in this study). In order to estimate the background from surface event leakage a ratio of the number of good events that are in the fiducial volume and lie in the nuclear recoil band to the number of good events that pass the radial cut was calculated for recoil energies between between 5 and 100 $\mathrm{keV}$. Two additional restrictions were imposed requiring that the yield be in the range between 0.1 and 0.85 (rough selection for surface events) and the maximum ionization energy measured on either of the sides was below $400 \mathrm{keV}$. If there are no true nuclear recoils in the data, this ratio tells us what fraction of beta events that pass the radial cut leak into the signal region. However, this data was taken at a laboratory on the surface of the earth and not shielded against neutrons. Hence it is expected to have true nuclear recoil events from cosmogenic and ambient neutrons. To arrive 
at the true leakage rate we would have to subtract this background. Unfortunately, this background is not well known. Since we are using the same data to compare two detector designs, the number of true nuclear recoils is the same for each. So instead of comparing the absolute discrimination we compare the performance of the iZIP and HiZIP configurations under the given experimental circumstances without background subtraction.

Let us call the ratio of apparent leakage (without background subtraction) in HiZIP and iZIP as 'uncorrected surface event leakage' (USEL) for the purpose of this study. The USEL of 8 configurations of HiZIP (based on bias polarity, side the radial cut is applied and the side for the phonon sensor) were calculated along with the USEL for two bias polarities of the iZIP detector and are shown in table 6.1. The radial cut applied in the case of iZIP is a 'logical OR' of side 1 and side 2 radial cuts, i.e. the events which pass the radial cut on at least one side are retained. The most noticeable trend that can be seen here is that the USEL of HiZIP configurations with phonon sensors on side 1 are smaller than those with phonon sensors on side 2. This effect mainly comes from the fact that most surface events in this dataset are on side 1 and the under-estimation of phonon energy by phonon sensors on side 2 makes the yield for these events (black) higher and hence away from the NR band (see figure 6.18). True nuclear recoils in the bulk do not suffer very much from this effect and the numerator of the USEL ratio remains nearly the same whereas the denominator in the USEL ratio (the total number of 'betas' considered in this calculation) is considerably smaller due to the big portion of band moving up and out of the yield range 0.1 to 0.85. However a more thorough analysis would also account for events below the NR band which can potentially move into the NR band and affect the numbers we have 
calculated. The number of total events in the beta band is smaller in the cases when phonon sensor is on side 2 due to the underestimation of the phonon energy which results in an increased yield as discussed earlier (see figure 6.3). Due to the oblique propagation of electrons, this effect is much stronger when side with the phonon sensors collect electrons.

The time dependence of electron and hole signals for surface events was not studied and also the radial cut on side 2 selects fewer surface events than the radial cut on side 1 , hence it is hard to draw a conclusion from these numbers on which hardware configuration is better. However, it can be seen that for certain configurations the performance of the HiZIP is comparable to the iZIP.

A better way to compare the expected leakage of HiZIP and iZIP is to find how many events that lie in the iZIP's beta band and happen inside the fiducial volume leak into the NR band in the HiZIP analysis. It was found that none of these events leaked into the HiZIP NR band. This testifies that the discrimination performance of the HiZIP to be comparable to the iZIP detector. We lose about $5-15 \%$ of the fiducial volume in the signal region but manage to simplify the detector and achieve discrimination performance comparable to the iZIP. A real detector wired in this configuration would also offer the possibility to detect MISS events. It needs to be examined if this is a worthy trade off in the long run. 
Table 6.1: G48 HiZIP uncorrected surface event leakage estimates (USELs) for various HiZIP configurations. The HiZIP has phonon sensors on either side 1 or side 2 while the iZIP has phonon sensors on side 1 and 2 ( $1^{\text {st }}$ column). The side on which radial cut was applied is listed in $2^{\text {nd }}$ column. 'Pass events' are the events in the signal region while 'total events' represent the good events in the beta sample subject to radial cut. Refer to text for details.

\begin{tabular}{|c|c|c|c|c|c|}
\hline $\begin{array}{l}\text { Phonon } \\
\text { Sensor }\end{array}$ & $\begin{array}{l}\text { Radial } \\
\text { Cut }\end{array}$ & $\begin{array}{c}\text { Bias } \\
\text { Side } 1\end{array}$ & Pass Events & $\begin{array}{l}\text { Total Events } \\
\qquad\left(\mathrm{x} 10^{4}\right)\end{array}$ & $\begin{array}{c}\text { USEL }\left(\mathrm{x} 10^{-4}\right) \\
\text { (UL at } 90 \% \mathrm{CL})\end{array}$ \\
\hline \multirow{4}{*}{ Side 1} & \multirow{2}{*}{ Side 1} & + & 42 & 8.58 & 6.33 \\
\hline & & - & 53 & 8.48 & 7.86 \\
\hline & \multirow{2}{*}{ Side 2} & + & 39 & 9.25 & 5.51 \\
\hline & & - & 59 & 9.16 & 8.00 \\
\hline \multirow{4}{*}{ Side 2} & \multirow{2}{*}{ Side 1} & + & 45 & 4.02 & 14.4 \\
\hline & & - & 34 & 0.42 & 107 \\
\hline & \multirow{2}{*}{ Side 2} & + & 41 & 4.41 & 12.1 \\
\hline & & - & 38 & 0.56 & 88.6 \\
\hline \multirow{2}{*}{ Side $1 \& 2$} & \multirow{2}{*}{$1^{\prime} \mathrm{OR}{ }^{\prime} 2$} & + & 44 & 8.77 & 6.45 \\
\hline & & - & 40 & 7.84 & 6.64 \\
\hline
\end{tabular}



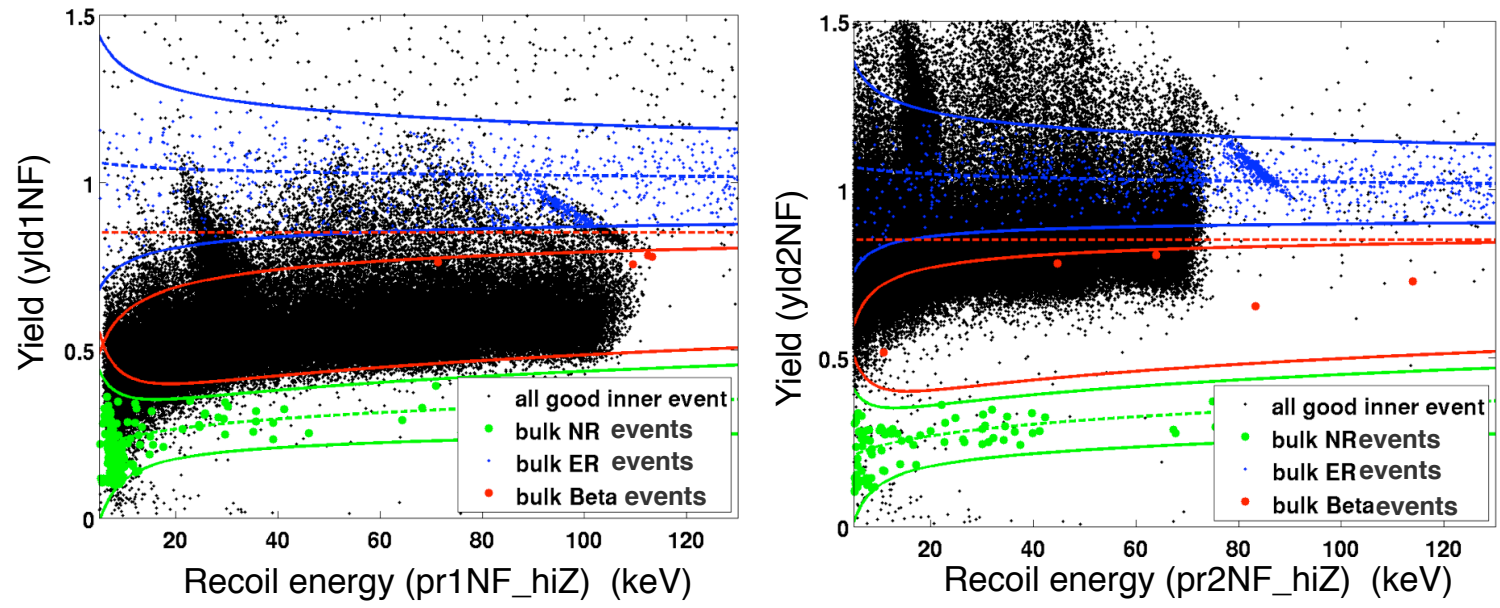

Figure 6.18: Leakage of beta events passing the charge symmetry cut into the signal region for phonon sensor on side 1 (left plot) and on side 2 (right plot). For both plots side 1 has a positive bias and the radial cut on side 1 is used. It can be seen that the population of green events is smaller at low energies when the phonon sensor is on side 2 . 


\section{Chapter 7}

\section{G16K HiZIP}

The G16K detector is an iZIP detector that has been rewired as a HiZIP device. We used this device to investigate in how far the results of the G48 studies described in chapter 6 can be achieved with a real HiZIP. This detector was the first iZIP-like detector operated at the Queen's detector test facility. The following section describes the detector, the experimental setup as well as data acquisition and processing. Section 7.2 will discuss the analysis and findings.

\subsection{Operating the G16K detector at SuperCDMS Queen's Test Facility}

The G16K is a rewired iZIP detector. The phonon sensors on side 1 have been connected together and converted into a charge channel. It has phonon sensors on side 2 alone. It has a single charge sensor on side 2 and three charge sensors on side 1 (see figure7.1). The single charge sensor on side 2 spans the entire side 2 surface 
and is called $\mathrm{Q}_{2}$. Side 1 not only has $\mathrm{Q}_{\text {inner }}$ and Qouter sensors just as in the iZIP detector, but also an additional charge sensor interleaved with $\mathrm{Q}_{\text {inner }}$ and $\mathrm{Q}_{\text {outer }}$ and spanning the entire surface called $Q_{p}$. For the reasons mentioned earlier in chapter 5. the new charge channel $\mathrm{Q}_{p}$ obtained by replacing phonon sensors on side 1 is not used in this study. This does not stop us from making a fair comparison with the G48 discrimination, though it must be noted that the detector is not used to its full potential here.

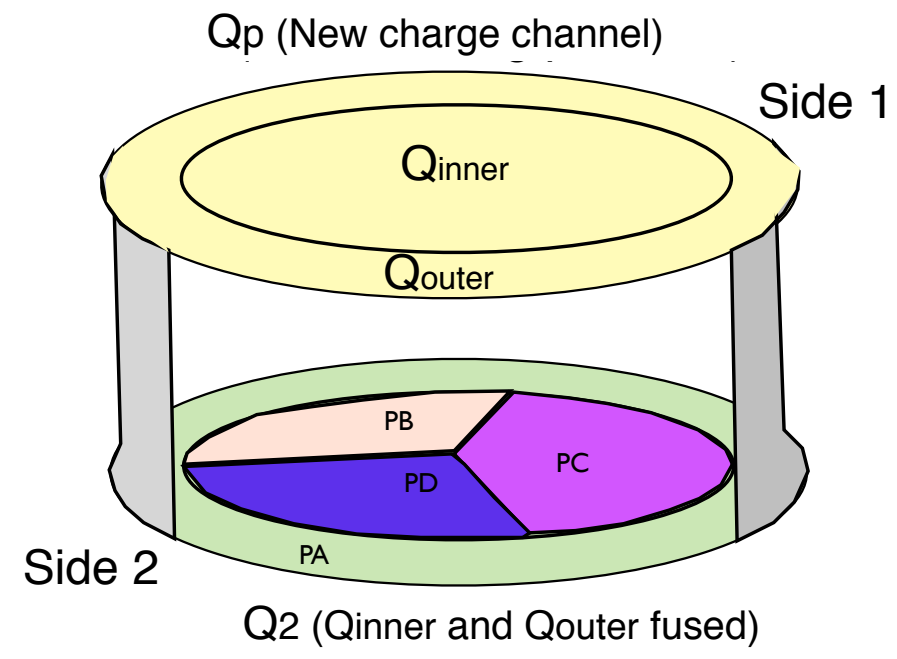

Figure 7.1: A schematic of the G16K detector. Side 1 has three charge electrodes $\mathrm{Q}_{\text {inner }}, \mathrm{Q}_{\text {outer }}$ and $\mathrm{Q}_{p}$, whereas side 2 has a single charge channel $\mathrm{Q}_{2}$ and four phonon channels PA, PB, PC and PD. $\mathrm{Q}_{p}$ is interleaved with $\mathrm{Q}_{\text {inner }}$ and $Q_{\text {outer }}$ sensors and spans the side 1 surface.

In order to read out all the signals from the G16K detector we use two DCRC readout boards and synchronize the clocks on the two boards. The DAQ system allows us to read all the channels every time there is a trigger on a channel on either 
board. A coincidence time of $200 \mu$ s between triggers is allowed for each event. The 'look-back time' setting on the DAQ graphical user interface allows us to record the 'pre-pulse baseline' for each event. The different settings for the readout electronics and recording data can be made through the DAQ software as described in chapter 3.

\subsubsection{Data acquisition and processing}

Due to the de-neutralization problem discussed in chapter 3, we can take data continuously for about 60 seconds after which the charge signal is unacceptably degraded. Hence we have to alternate every minute of data acquisition with flashing which frees up trapped charges and resets the detector to a charge-neutral state. The DAQ software is equipped to run in an automation mode where time intervals can be set for data acquisition, LED flashing and cooling time, repeating until stopped. This reduces a considerable amount of manual work that would be required otherwise. However, the amount of downtime required for cooling down after every flash is large and we end up with only an hour of good data for every 24 hours of data taken in the automation mode. This reduces the efficiency of data acquisition but it is necessary to gather sufficient statistics for a counting experiment of the kind pursued here.

The flashing and cool down periods were timed so that the detector was always at the same steady temperature while data was taken. Problems in the SQUID readout for the phonons could cause it to go out of operating range, hence we had to ensure proper operation throughout data acquisition. A schematic of the setup with the G16K detector and internal sources installed can be seen in figure 7.2 . The ${ }^{210} \mathrm{~Pb}$ source is used on the bottom side (side 1) while the ${ }^{241} \mathrm{Am}$ source is housed in the 
source holder on the top of the detector (side 2). These sources are referred to as internal sources and cannot be accessed once the detector is set inside the cryostat and cooled down. Radioactive sources which illuminate the detector from outside the cryostat are referred to as external sources.

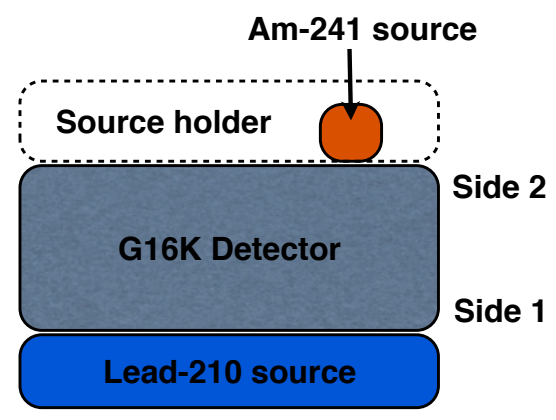

Figure 7.2: A schematic of the setup used to take data (shows internal sources only). Phonon sensors are on side 2 .

For the G16K HiZIP surface event discrimination studies three kinds of data sets were taken, they are:

- A surface event sample which has no external sources and a significant number of surface events from the ${ }^{210} \mathrm{~Pb}$ source.

- Bulk gamma events sample in order to define the electron recoil band. An external ${ }^{133} \mathrm{Ba}$ source was used for this.

- Bulk neutron events sample in order to define the nuclear recoil band. An external Americium-Beryllium neutron source was used for this.

For all the data used in this study, the ${ }^{210} \mathrm{~Pb}$ and ${ }^{241} \mathrm{Am}$ sources were always 
present. However, the charge symmetry constraint allows us to select events happening explicitly in the bulk for the gamma and neutron datasets. Dr. Kamaev and C. Crewdson adapted the CDMS data processing tools so they could be used efficiently to process the acquired data. The final data analysis is performed using the MATLAB based 'CDMS Analysis Package' (CAP).

\subsection{G16K Data analysis}

The surface event discrimination study follows the same basic steps as in the case of the G48 analysis discussed in chapter 6. Once the data are processed, some preliminary diagnostic checks are done to ensure nothing went wrong during data acquisition - All data sets passing these checks are complied into a list of good data sets available for the study. Vast amounts of data were taken. However, due to bad data quality, not all data are usable. Only side 1 positive polarity data are used in this study. The positive polarity data includes one dataset for each kind listed above, allowing us to do the discrimination study, albeit with smaller statistics than we had hoped for. The following section describes the development of data quality cuts used in this analysis.

\subsubsection{HiZIP data quality cuts}

New data quality cuts were developed considering the different problems specific to Queen's test facility in addition to some cuts that are commonly used in CDMS data analysis. 


\section{Phonon $\chi^{2}$ cut}

The phonon $\chi^{2}$ cut is defined on the optimal filter phonon quantities in a similar manner as discussed in chapter 6. It was not used as a quality cut in the final analysis due to considerable overlap in selection with other quality cuts. However, the phonon $\chi^{2}$ cut proved to be a very useful tool in the initial stages of the analysis to check data quality, to clean the data sample crudely but quickly during the definition of other phonon based quality cuts and for calibration.

\section{Charge $\chi^{2}$ cut}

The charge $\chi^{2}$ cut is defined in a similar way as the phonon $\chi^{2}$ cut. A quadratic polynomial is used to set the upper bound on the $\chi^{2}$ vs. ionization energy plane. Figure 7.3 shows an example of the implementation of the cut on the charge channel $\mathrm{Q}_{2}$. 


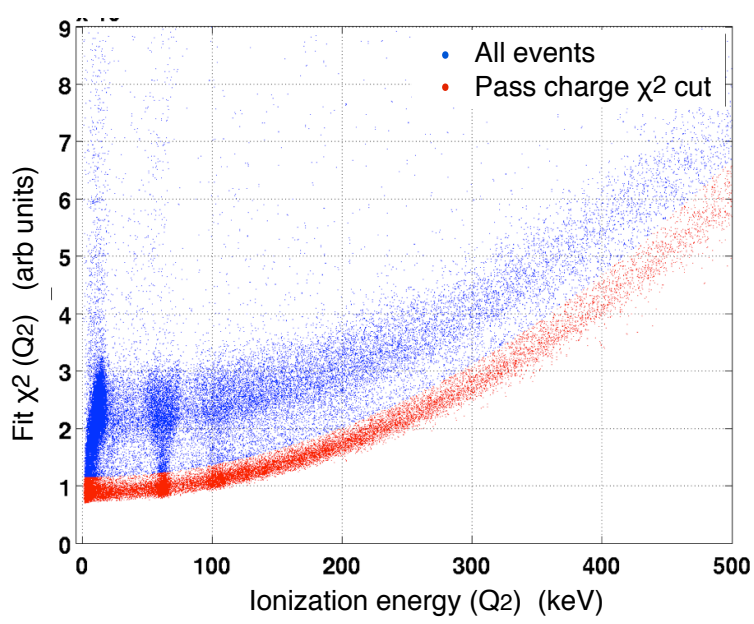

Figure 7.3: Charge $\chi^{2}$ cut on the $\mathrm{Q}_{2}$ charge channel. Among the discarded events is also a band of events with elevated $\chi^{2}$ which closely follows the shape of events passing the cut, these events mostly come from periods of elevated noise. Figure created by Chase Crewdson 93.

\section{Phonon pileup cut}

The pileup cut is based on the number of fast rising edges in a phonon trace. The number of fast rising edges are calculated for each phonon channel trace and for all events. They indicate the number of fast rising edges in the trace window for each phonon trace. The algorithm to identify pileup events works as follows: all the phonon channel traces for all channels are smoothed and their derivatives are calculated. A threshold on the derivative is determined empirically to ensure only pulses that have the derivative of their rising edge above the threshold are selected. Each of the four phonon traces for each event is scanned to find the number of times this threshold is crossed. If any of the traces' derivatives have more than one threshold crossings 
the algorithm checks if the first threshold crossing is between 400-800 time bins since the main pulses' rising edge is set well within this interval. It also checks if any of the consecutive crossings are separated by more than 250 time bins. This condition makes the algorithm insensitive to small variations in the pulse shape close to the peak of the pulse. This is necessary since the pulse shapes have some inherent variation corresponding to type and location of the event in the detector and often resemble a combination of a fast pulse and a slow pulse. The flowchart for the algorithm is shown in figure 7.4. The number of bins used in the moving average and the threshold for detecting the rising edges are set empirically.

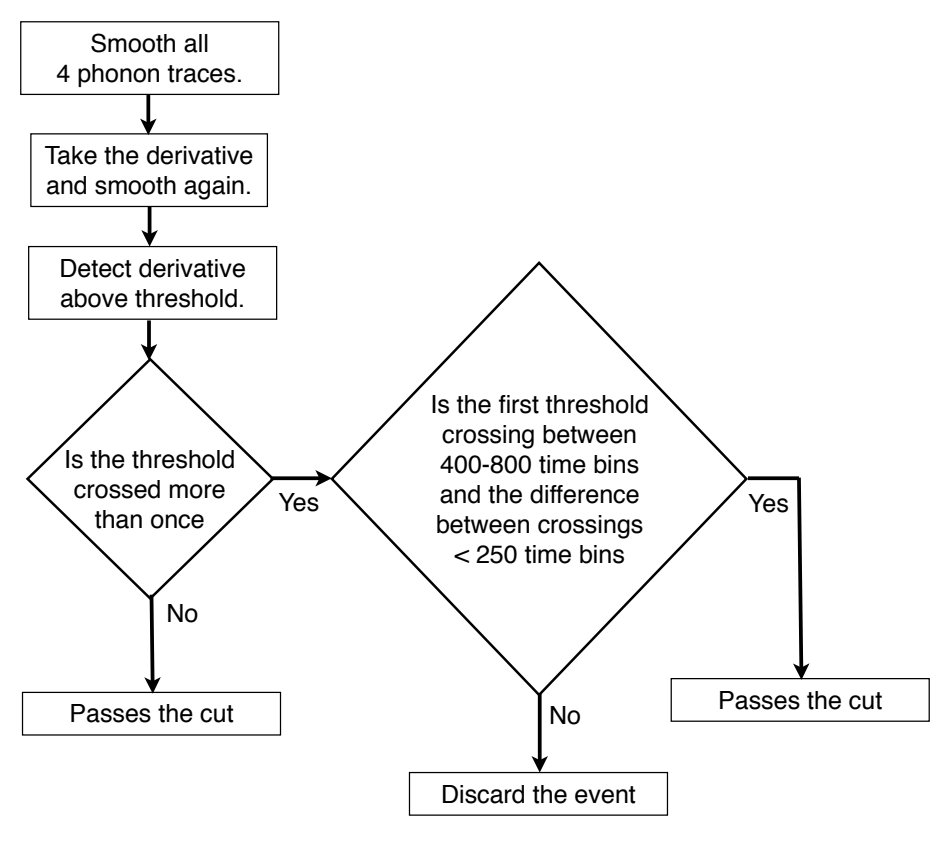

Figure 7.4: The algorithm used to identify and discard pileup events where more than a single pulse is recorded in the event window.

This algorithm is very efficient at picking out pileup events where there is more 
than a single pulse in the trace window $(\sim 3.3 \mathrm{~ms})$. However, the parameters need to be tuned every time the calibration for a channel is changed during different data acquisition runs, making the process time consuming. The algorithm is not sensitive to 'sloped pulses' (pulses which sit on the tail of a previous pulse) and we use a cut on the phonon traces' pre-pulse baseline similar to the one described in chapter 6 to remove this class of events.

The passage fraction for the pileup cut in different energy bins is plotted below in figure 7.5. It was ensured that the pileup cut neither removes too many events at lower energies nor does it remove certain kinds of events preferentially over others except real pileup events of course.

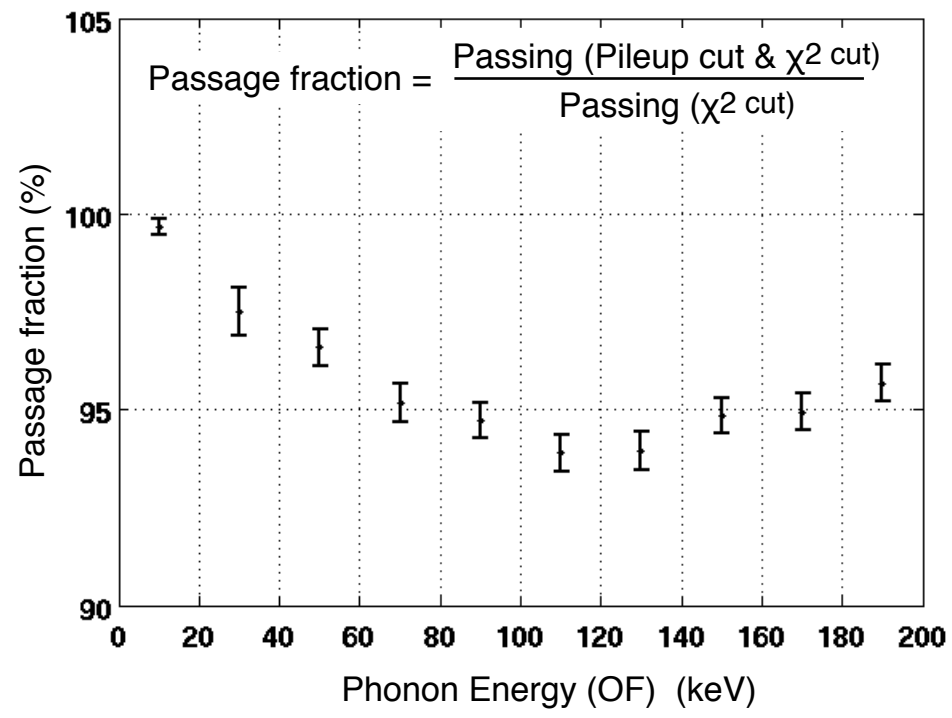

Figure 7.5: Passage fraction of the pileup cut with respect to the $\chi^{2}$ cut as function of phonon energy $(\mathrm{OF})$. The $\chi^{2}$ cut is very efficient at removing pileup events at low energies which leads to an apparent increase in the pileup passage efficiency. 


\section{A cut to remove replica events}

The data acquisition system is designed to be dead-time free, hence if multiple trigger conditions are satisfied the event is recorded multiple times. This results in replica events recorded as distinct events in the raw data (see figure 7.6). The replica events need to be removed explicitly during the analysis if we are to perform a counting experiment. They are mostly found to be separated by one to eight events from the original event in terms of the 'Event Number' (an index assigned to in the order they are recorded on disk).
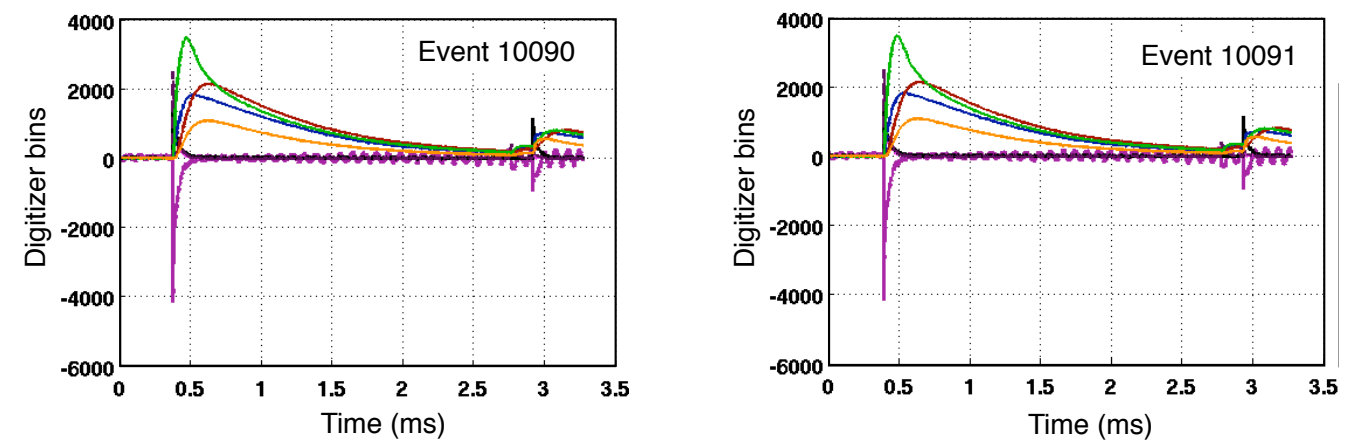

Figure 7.6: An example of two consecutive events being replicas. The different traces shown correspond to different channels, both charge and phonon channels are included. Coincidentally, this event is also a pileup event.

The algorithm used to remove these events compares the pulse maximums of each phonon trace (all channels) of a given event to those of the next eight events and removes an event as a replica of the original if the pulse maximums on all channels match with the original to within a small degree of uncertainty. The small uncertainty window on the pulse maximums comes from the specifics of the DAQ. An upper limit of this uncertainty was determined empirically and input to the algorithm. The 
algorithm removes the replica events with a very high accuracy.

\section{A cut on phonon pre-pulse baseline standard deviation.}

A cut on the pre-pulse phonon baseline standard deviation is very efficient at removing pulses with sloped baselines. Gaussian fits were made to the distribution of pre-pulse baseline standard deviations. A cut was placed with a lower bound of $4 \sigma$ below the gaussian mean and an upper bound of $2.5 \sigma$ above the gaussian mean. Where $\sigma$ is the standard deviation of the gaussian fit to the distribution (see figure 7.7). The distribution should ideally be Gaussian for white noise (i.e.no sloped pre-pulse

baselines). However, due to an increased number of events with sloped baselines coming from pileup events, the distribution is not exactly Gaussian. A cut at $2.5 \sigma$ upper bound is found to remove most of these pileup events, while a lower bound of $3 \sigma$ ensures that we do not include events in which any of the phonon channels are not operational (i.e.the pre-pulse standard deviation is 0 ). An event failing the cut on at least one of the four phonon channels is removed. 


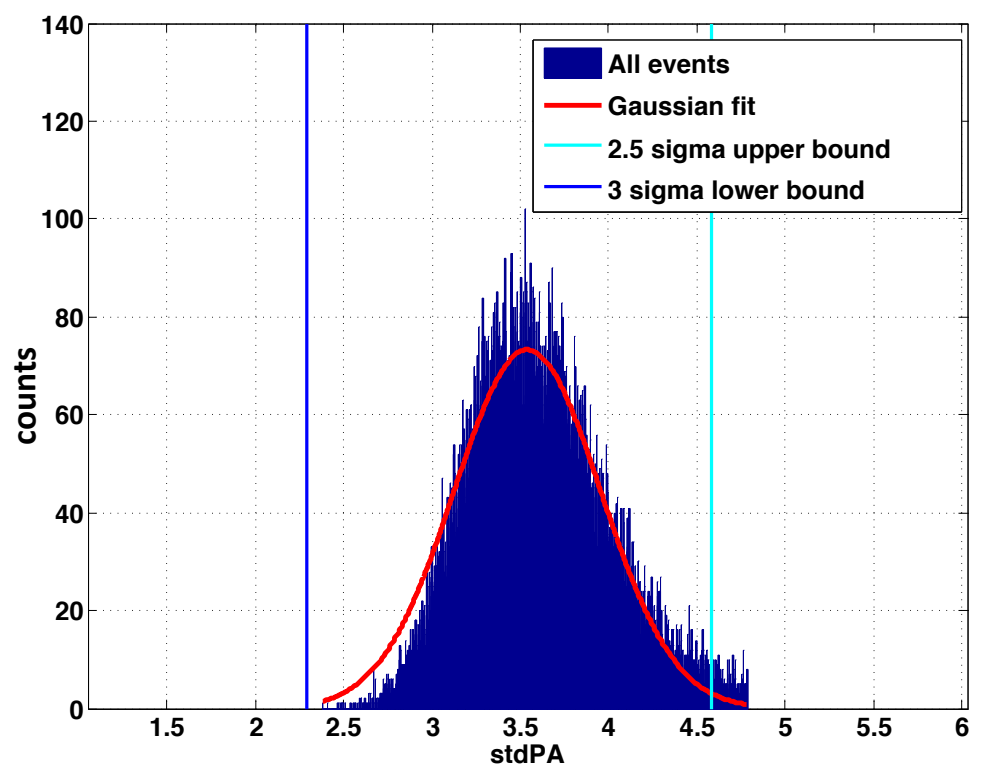

Figure 7.7: The histogram of phonon pre-pulse baseline standard deviation for channel PA. An upper limit is placed at $2.5 \sigma$ of the gaussian fit.

\section{Charge signal stability cut (neutralization cut)}

Due to the de-neutralization problem discussed in chapter 3 we have to remove events which happen when the detector is in a bad neutralization state. In order to do this we plot the charge signal vs time on each channel and look for degradation of the charge signal, and then manually set a time limit up to which we should accept events (see figure7.8). The smallest time limit among all the channels defines the time limit for the cut. 


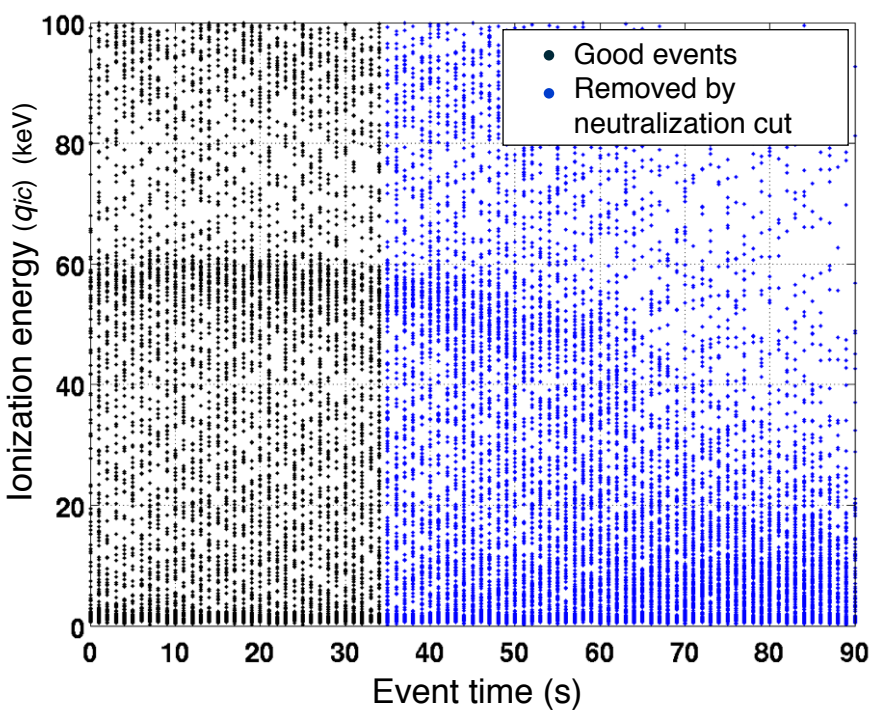

Figure 7.8: The neutralization cut on $Q_{i n n e r}$ charge channel. The calibrated energy on $\mathrm{Q}_{\text {inner }}$ is called qic. The event time is binned in seconds from the start of data acquisition . Figure created by Chase Crewdson [93].

\section{Bad periods cut}

In our data taken at the Queen's SuperCDMS test facility we find periods of high noise which affect our data quality to an unacceptable degree and need to be removed. These periods are noticeable in the charge $\chi^{2}$ (see figure 7.3 ) and are for the most part removed by the charge $\chi^{2}$ cut. However, to ensure we have removed all the events happening in these periods of high noise, we discard all data taken during these times. To do this we plot a quantity 'Badness factor' (charge $\chi^{2}$ normalized to charge energy) vs time and manually place a cut on time to remove the bad periods. These periods are also quite evident in the OF reconstructed charge energies (see figure 7.9 . 


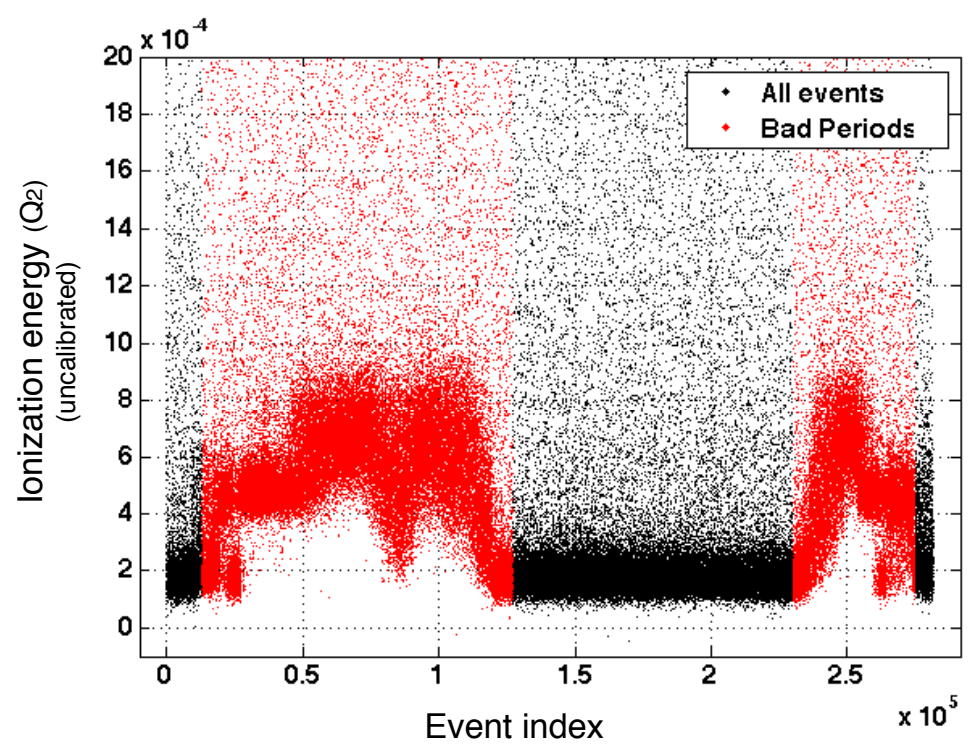

Figure 7.9: An example of bad periods seen in the uncalibrated charge OF quantity of charge channel $\mathrm{Q}_{2}$. Bad periods marked in red are removed by this cut. Event index is the count of the event recorded chronologically from the start of data acquisition .

\subsubsection{Charge Calibration}

The charge calibration had to be performed on the three charge channels $\mathrm{Q}_{2}, \mathrm{Q}_{\text {inner }}$ and $Q_{\text {outer }}$ for the three positive polarity data sets (gamma, beta-only and neutrons). We use the $356 \mathrm{keV}$ spectral line from ${ }^{133} \mathrm{Ba}$ gammas for calibration. Firstly the 356 keV gamma peak from bulk events is identified in the $\mathrm{Q}_{2}$ spectrum and a Gaussian fit is made to the peak. The mean of the gaussian is scaled accordingly to calibrate $\mathrm{Q}_{2}$. The bulk $356 \mathrm{keV}$ events are selected on $\mathrm{Q}_{2}$ and used to calibrate $\mathrm{Q}_{\text {inner }}$ and $\mathrm{Q}_{\text {outer }}$ relative to each other. These events form an off-diagonal band in the $\mathrm{Q}_{\text {inner }}-\mathrm{Q}_{\text {outer }}$ space due to different fraction of charge sharing between $Q_{\text {inner }}$ and $Q_{\text {outer }}$ (see figure 
7.10). After this relative calibration $\mathrm{Q}_{\text {inner }}$ and $\mathrm{Q}_{\text {outer }}$ are added and a Gaussian with a linear background is fit to the peak corresponding to the $356 \mathrm{keV}$ line in the spectrum of this new quantity $\mathrm{Q}_{1}$. The mean of the gaussian fit is set to $356 \mathrm{keV}$ to calibrate $\mathrm{Q}_{1}$. The calibrated $\mathrm{Q}_{1}$ and $\mathrm{Q}_{2}$ quantities are called $\mathrm{q}_{1 c}$ and $\mathrm{q}_{2 c}$ respectively in this analysis.

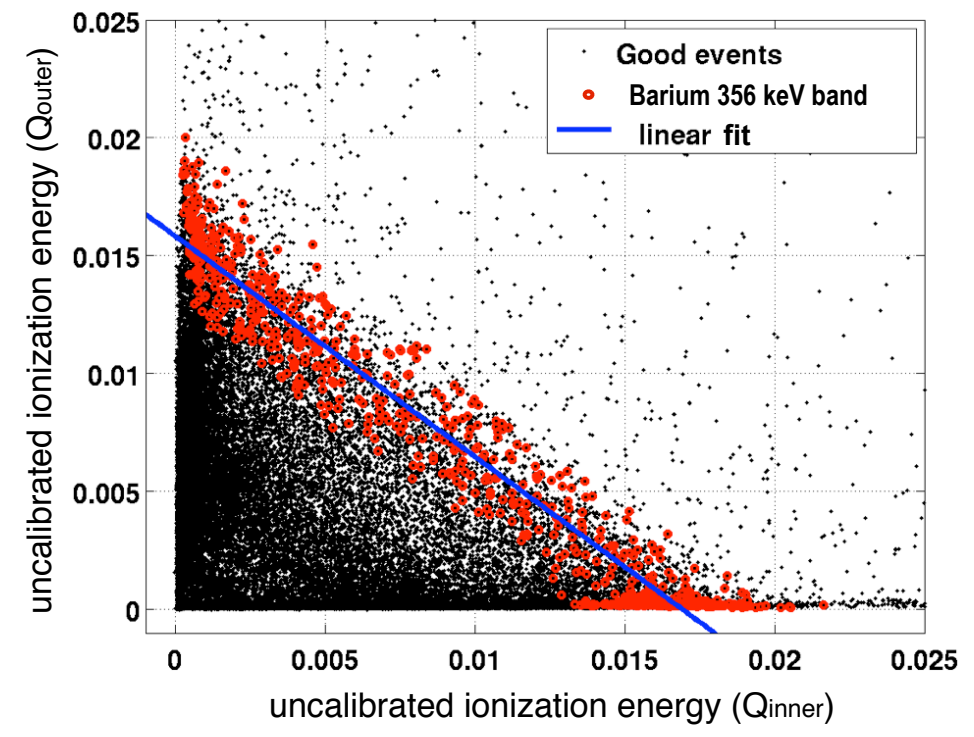

Figure 7.10: $Q_{\text {inner }}$ and $Q_{\text {outer }}$ calibration by fitting a line to the off-diagonal band composed of the $356 \mathrm{keV}$ gamma peak which is identified and selected on the $\mathrm{Q}_{2}$ channel. The selection is confirmed with the phonon energy spectrum.

The gamma, beta-only and neutron datasets were taken on consecutive days under similar conditions and there was no evidence of a shift in the calibration of charge channels. Hence, the calibration done using $356 \mathrm{keV}$ bulk barium gammas was applied to all three datasets. As an example, the calibrated and uncalibrated charge spectra 
of channel $\mathrm{Q}_{2}$ are shown in figure 7.11 . The $60 \mathrm{keV}$ and gamma peak and a few other gamma peaks close to $100 \mathrm{keV}$ (unresolved) from Am-241 internal source are common to all datasets. The $356 \mathrm{keV}$ gamma line from ${ }^{133} \mathrm{Ba}$ internal source is clearly visible on the 'Barium' dataset.
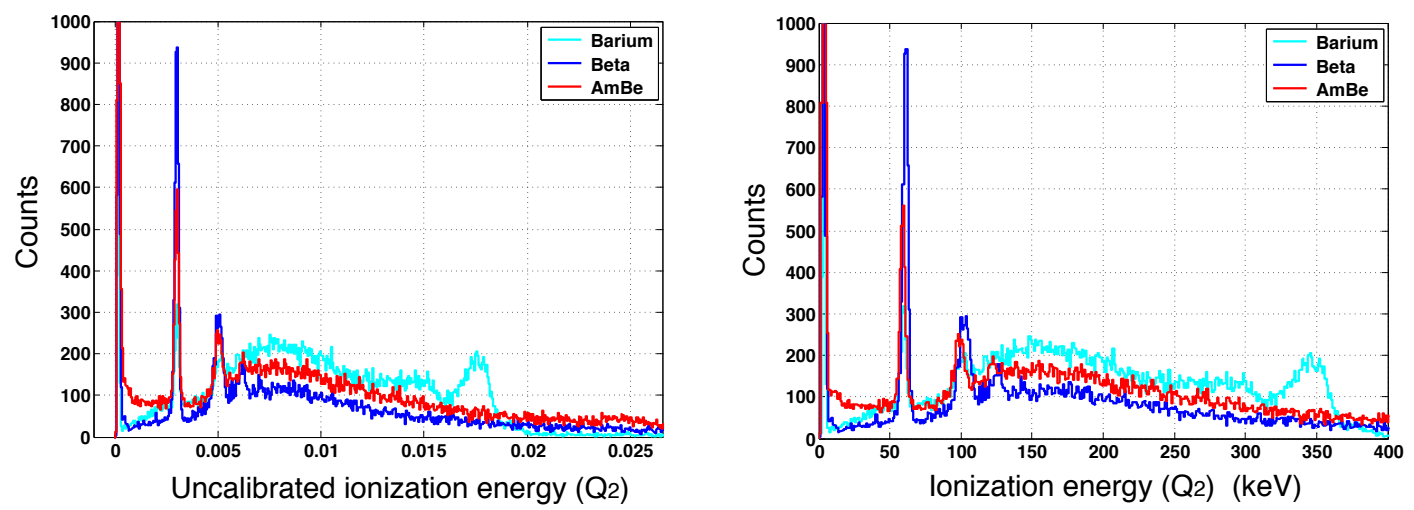

Figure 7.11: Uncalibrated (left) and calibrated (right) charge spectra of channel $Q_{2}$ for all the three datasets used. 'Barium' is the dataset with taken with an external ${ }^{133} \mathrm{Ba}$ source, the 'Beta' dataset is taken with no external sources and the 'AmBe' dataset is taken with an external Am-Be neutron source.

\subsubsection{Phonon Calibration}

The phonon calibration also involves two stages. Since our phonon energy estimators are constructed out of the phonon information from all four phonon sensors (on side 2 ), we have to first calibrate them relative to each other. The absolute calibration is then performed and the luke correction is applied to find the true recoil energy. 


\section{Relative calibration of phonon sensors}

As discussed in chapter 6, the tail of the phonon pulse has position independent energy information and the phonon energy is absorbed uniformly across all sensors. This allows us to match the tail of the phonon pulse on each of the channels in order to calibrate the sensors relative to each other. In order to do this we select a sample of good events and then plot the 'late integral' distributions (integral of the phonon trace from bins 1280 to 1980) of $\mathrm{PB}, \mathrm{PC}$ and $\mathrm{PD}$ versus PA for these events (see figure 7.12 for an example). Linear fits are made to the distributions and the relative calibration factors are calculated with respect to PA by setting the slope of the fit line to 1 . Figure 7.13 shows an example event before and after the relative calibration.

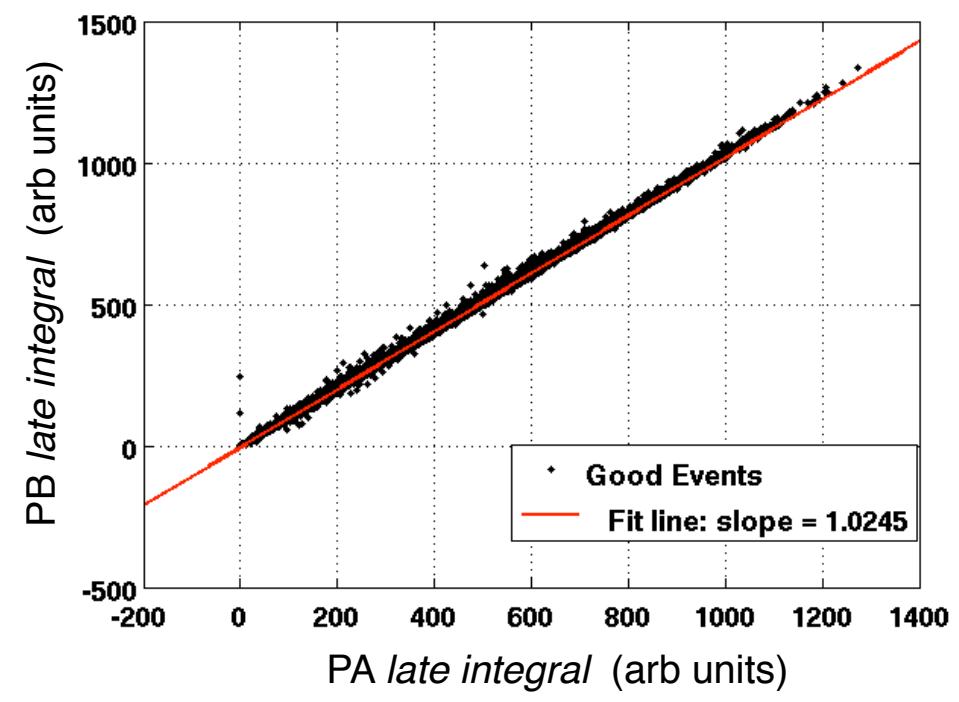

Figure 7.12: A plot of late integral distributions of channel PA vs PB for a sample of good events. 

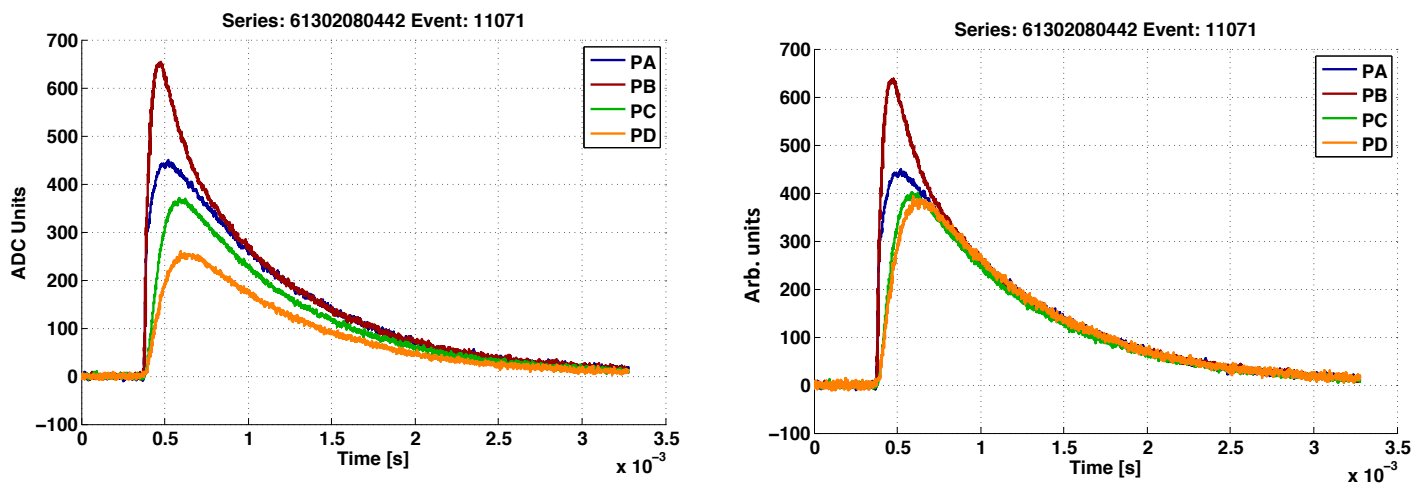

Figure 7.13: Phonon traces of an event before (left) and after (right) relative calibration.

\section{Absolute calibration of phonon energy and Luke correction}

The NSOF algorithm takes the relative calibration factors and the raw data and produces a phonon energy estimate for each event. These reconstructed energies are calibrated using the $356 \mathrm{keV}$ peak from bulk ${ }^{133} \mathrm{Ba}$ gammas. First a raw yield is calculated as a ratio of $\mathrm{q}_{\text {summax }}$ (maximum of $\mathrm{q}_{1 c}$ and $\mathrm{q}_{2 c}$ ) to the uncalibrated energy estimator from the NSOF algorithm. The raw yield is calibrated to 1 for bulk gamma events from the $356 \mathrm{keV}$ peak and called ytc. The phonon energy is calibrated using the calibration factor from this last step and called ptc. However, from chapter 2 we know that the total measured phonon energy is the sum of recoil energy $\left(E_{\text {recoil }}\right)$ and the Luke-phonon contribution $\left(\mathrm{E}_{\text {Luke }}\right)$. The 'Luke-correction' (Luke-phonon contribution) is obtained by using equation 2.5 and substituting $\mathrm{V}_{\text {bias }}$ $=3 \mathrm{~V}$ and $\epsilon=3 \mathrm{eV}\left(2 \mathrm{~V}_{\text {bias }}\right)$. We find that the 'Luke correction' in this case is just the sum of $\mathrm{q}_{1 c}$ and $\mathrm{q}_{2 c}$.

$$
\mathrm{E}_{\text {Luke }}=\left(\mathrm{q}_{1 \mathrm{c}}+\mathrm{q}_{2 \mathrm{c}}\right)
$$


For a perfectly bulk event symmetric bulk event $q_{1 c}=q_{2 c}$. Since the recoil energy for bulk electron recoils is the same as the measured ionization energy, the total phonon energy is thus $3 \times \mathrm{E}_{\text {recoil }}$. Since earlier we set ptc equal to $\mathrm{E}_{\text {recoil }}$ for bulk gammas, we can calculate $\mathrm{E}_{\text {recoil }}$ for all events as:

$$
\mathrm{E}_{\text {recoil }}=3 \times \mathrm{ptc}-\left(\mathrm{q}_{1 \mathrm{c}}+\mathrm{q}_{2 \mathrm{c}}\right)
$$

However, the yield $y t c$ is not 'Luke-corrected'. The 'Luke-corrected' yield (or simply 'yield') is calculated as:

$$
\operatorname{yrc}=\frac{q_{\text {summax }}}{\mathrm{E}_{\text {recoil }}}
$$

\subsubsection{Fiducial volume and radial cut efficiency}

The fiducial volume is again defined using the charge information as in the case of G48 analysis discussed in chapter 6. The charge symmetry cut and the radial cut together define the fiducial volume.

\section{Charge symmetry cut}

The charge symmetry cut $\left(\mathrm{cQ}_{\text {Sym }}\right)$ as introduced in chapter 6, selects bulk events and is defined in the same manner as discussed in chapter 6. It is made sure that the $c Q_{\text {Sym }}$ cut selects events which either produce a symmetric charge signal which is above the threshold on both sides $\left(\mathrm{Q}_{1}\right.$ and $\left.\mathrm{Q}_{2}\right)$ or events which produce a charge signal that is below the threshold on both sides (see figure 7.14). 


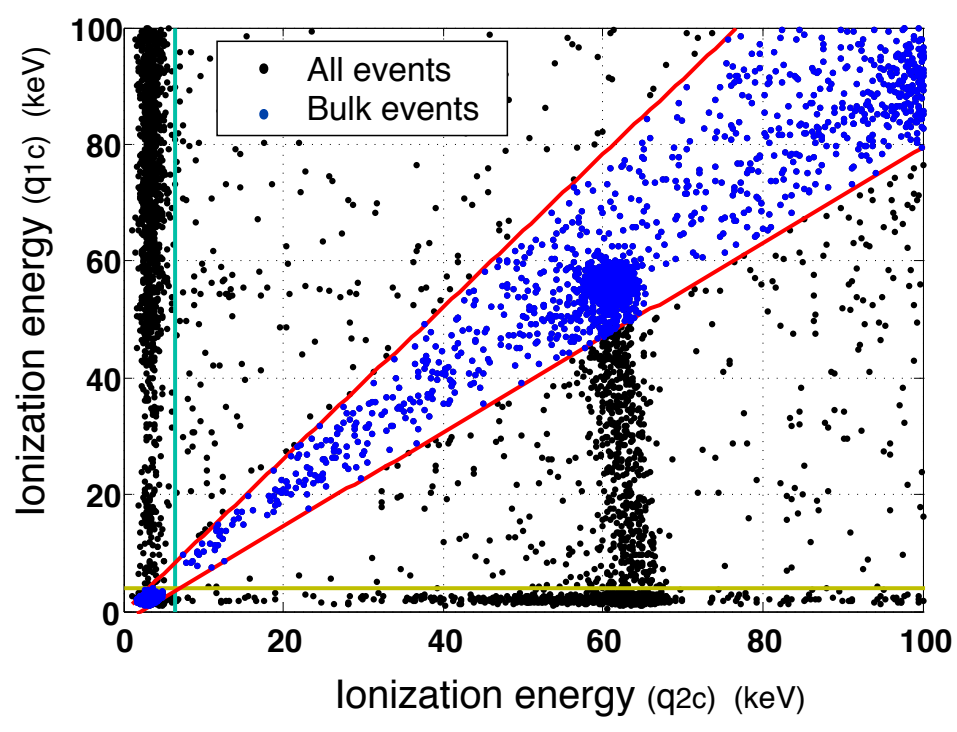

Figure 7.14: The G16K charge symmetry cut. The red lines mark the region of inclusion and the vertical and horizontal lines indicate the $6 \sigma$ noise threshold on $\mathrm{Q}_{2}$ and $\mathrm{Q}_{1}$ respectively.

\section{Radial cut to select inner events}

The radial cut is also defined in a similar manner as discussed in chapter 6. A more conservative cut was defined which includes only 'inner events' (i.e.events producing a signal only on $\mathrm{Q}_{\text {inner }}$ on side 1). This is due to the fact that the events happening close to the side-wall of this detector were not studied sufficiently at the Queen's test facility and we do not seem to gain very many events by allowing charge sharing between $Q_{\text {inner }}$ and $Q_{\text {outer }}$. The radial cut (cQin1) is shown in figure 7.15 on a plot of $\mathrm{q}_{i c}$ vs $\mathrm{q}_{o c}$ where $\mathrm{q}_{i c}$ and $\mathrm{q}_{o c}$ are the calibrated $\mathrm{Q}_{\text {inner }}$ and $\mathrm{Q}_{\text {outer }}$ energies. 


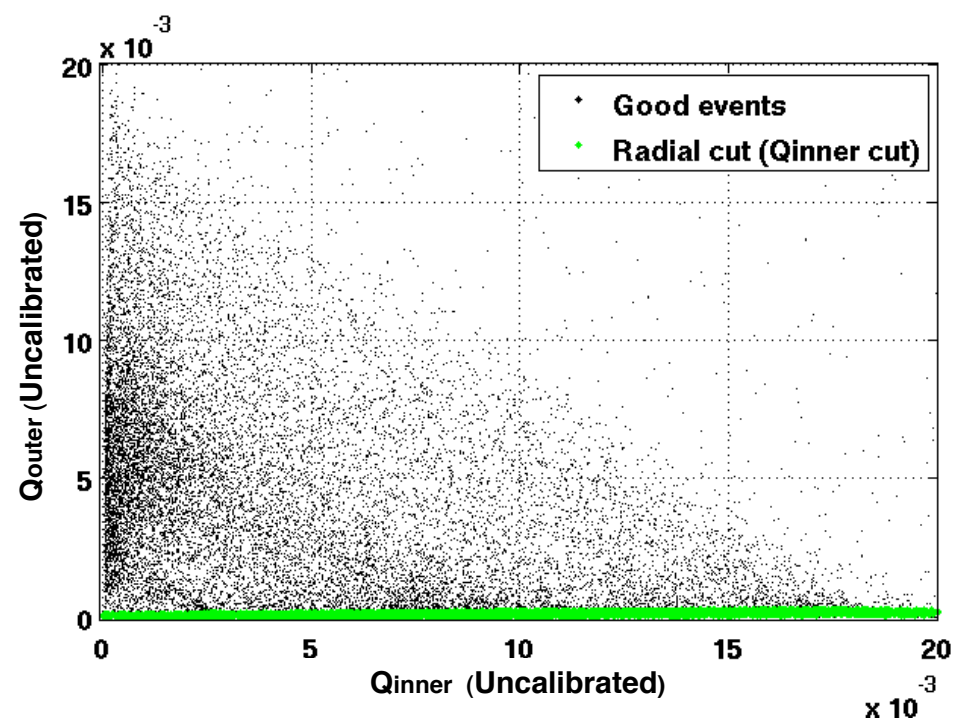

Figure 7.15: The G16K radial cut. It selects events producing no signal on the Qouter channel (marked in green).

The radial cut efficiencies were calculated for both ER and NR bands just as in chapter 6. The efficiency is defined as the fraction (in \%) of all good bulk events passing the radial cut. The efficiency, and in turn the fiducial volume itself, seems to decrease with increasing recoil energies in both NR and ER bands. The drop in efficiency is relatively less in the case of the ER band. For an event happening at a certain location in the detector the signal partitioning between $\mathrm{Q}_{\text {inner }}$ and $\mathrm{Q}_{\text {outer }}$ is fixed. At very low energies, the signal in $Q_{\text {outer }}$ is below its threshold despite the partitioning between $Q_{i n n e r}$ and $Q_{\text {outer }}$ and hence the event passes the radial cut. For an event at the same location, if the fraction of the signal seen by Qouter $_{\text {is }}$ higher than its threshold it gets rejected. Hence the fiducial volume is dependent on the energy of interaction and shrinks with increasing energy. Since the ionization signal for a NR event is about a third of the ionization signal for a ER event, the acceptance 
of the radial cut for a given recoil energy is naturally better for NR events.
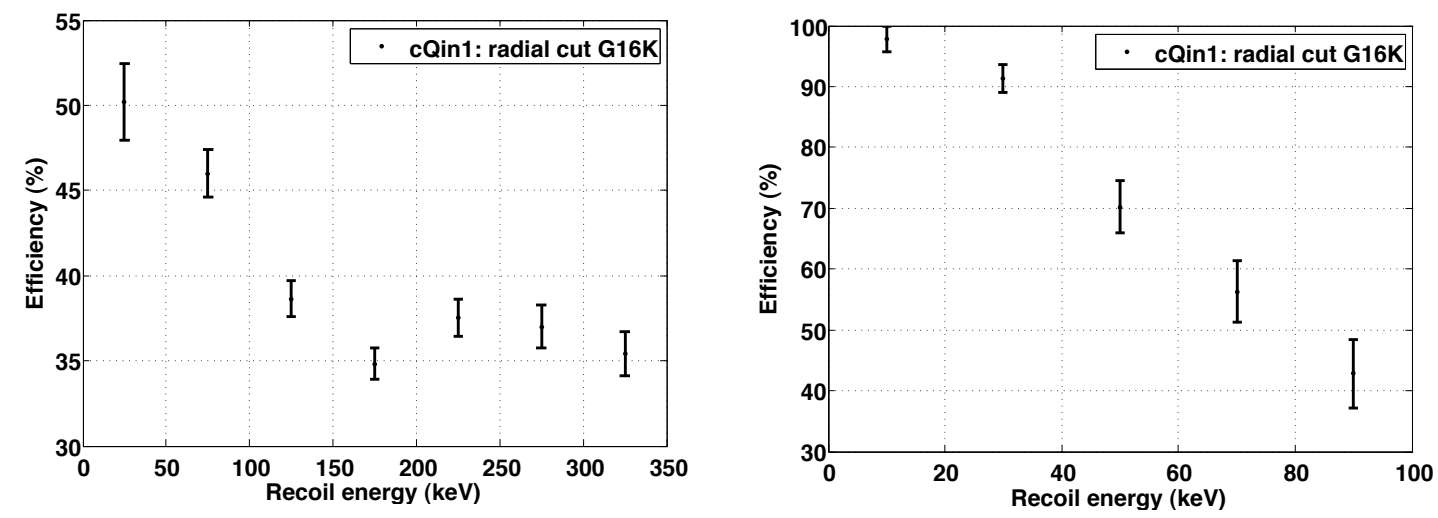

Figure 7.16: Radial cut efficiencies in different recoil energy bins for bulk ER events (left) from 'Barium' dataset and bulk NR events (right) from the 'AmBe' dataset. The bin width used is $50 \mathrm{keV}$ for ER events and $20 \mathrm{keV}$ for NR events.

\subsubsection{Defining yield bands}

The electron recoil (ER) band is defined using bulk events from the ${ }^{133} \mathrm{Ba}$ dataset. The nuclear recoil (NR) band is defined using bulk neutron events from the AmericiumBeryllium dataset. The 'beta band' is defined as the region $3 \sigma$ below the ER band mean and $3 \sigma$ above the NR band mean. These bands are shown in figure 7.17 . 


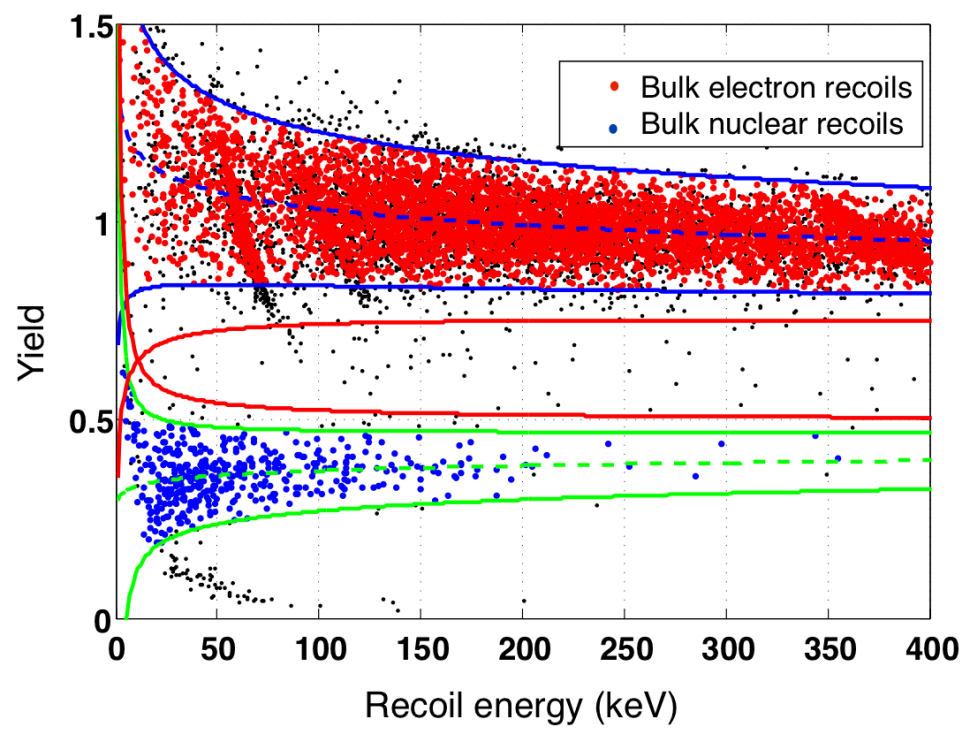

Figure 7.17: G16K yield bands. The blue lines demarcate the ER band, the green lines demarcate the NR band and the red lines demarcate the beta band.

\subsubsection{The charge threshold cut}

The charge threshold cut serves to remove events for which the charge signal on both $\mathrm{Q}_{1}$ and $\mathrm{Q}_{2}$ is consistent with noise to within $6 \sigma$ (standard deviations). A stringent limit of six standard deviations was chosen to be sure that very short bursts of noise which could affect the energy estimation, especially at lower energies, be avoided. Gaussian fits were made to the histograms of noise events (events with no pulses) for $\mathrm{q}_{1 c}$ and $\mathrm{q}_{2 c}$. The cut was then defined to exclude events which have both $\mathrm{q}_{1 c}$ and $\mathrm{q}_{2 c}$ below $6 \sigma$. The threshold cut was not used as a data quality cut and the noise events are retained throughout the analysis. The cut is used in the surface event leakage study. Figure 7.18 shows the events excluded by the threshold cut on the $\mathrm{q}_{1 c} \mathrm{vs}_{2 c}$ plane. 


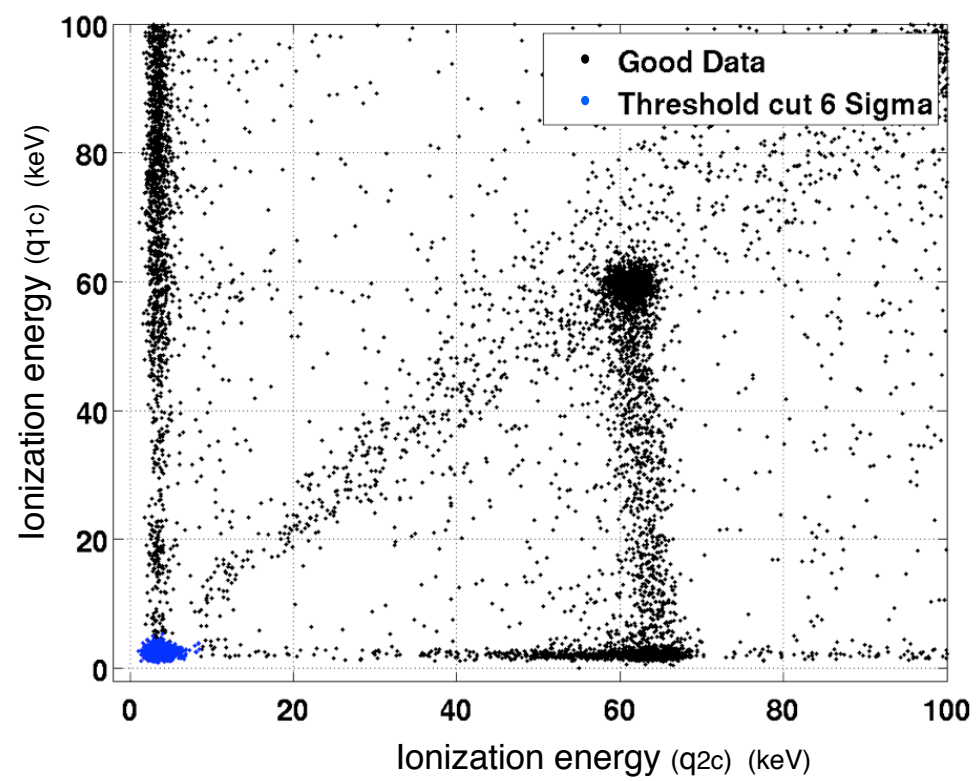

Figure 7.18: The G16K charge threshold cut removes events in blue. These events are within $6 \sigma$ above the mean on both $\mathrm{Q}_{1}$ and $\mathrm{Q}_{2}$ channels.

\subsubsection{Surface event leakage estimation}

The surface event leakage is estimated in the same way as for the G48 analysis. A surface event sample from the ${ }^{210} \mathrm{~Pb}$ beta source on side 1 was used for this study. The majority of events in this dataset come from ${ }^{210} \mathrm{~Pb}$ betas (low energy), ${ }^{210} \mathrm{Bi}$ betas (high energy), electrons from excited ${ }^{210} \mathrm{Bi}$ internal conversion, gammas from ${ }^{210} \mathrm{~Pb}$, gammas from the ${ }^{241} \mathrm{Am}$ internal source, alphas from ${ }^{210} \mathrm{Po}$ and some recoiling ${ }^{206} \mathrm{~Pb}$ nuclei. These ${ }^{206} \mathrm{~Pb}$ events occur when the ${ }^{210} \mathrm{Po}$ decays by emitting a high energy alpha away from the detector, sending the daughter nucleus $\left({ }^{206} \mathrm{~Pb}\right)$ recoiling toward the detector.

The analysis threshold is set by the lower bound of the beta band; this was found 
to be $11 \mathrm{keV}$. The energy regime for the analysis is 11 to $100 \mathrm{keV}$ in recoil energy. The HiZIP hardware configuration under investigation has phonon sensors on side 2. Side 1 has the $Q_{\text {inner }}-Q_{\text {outer }}$ separation (radial cut is applied on side 1 ) and is positively biased. The USEL is as defined in chapter 6. The number of events passing (numerator of USEL) and the total number of events (denominator of USEL) for this hardware configuration are shown in table 7.1 for both the G16K (at Queen's ) and G48 detectors (at UC Berkley). Since the passing events also have true nuclear recoil events from neutrons and we do not have a good estimate for the neutron rate, we do not attempt to account or correct for the neutron background, instead we state a 90 \% confidence level (CL) upper limit (UL) on the leakage treating the passing events as surface event leakage. It can be seen that the USEL is better in the case of G48 analysis.

Table 7.1: Uncorrected surface event leakage (USEL) estimates for the G16K detector and the G48 detector in the same hardware and bias configuration as G16K data used here. The column 'USEL' lists the 90\% confidence level (CL) upper limits (UL).

\begin{tabular}{|c|c|c|c|}
\hline Detector & Total events & Passing events & $\begin{array}{c}\text { USEL } \\
(\mathrm{UL} \text { at } \mathbf{9 0 \%} \mathrm{CL})\end{array}$ \\
\hline $\begin{array}{c}\text { G16K } \\
\text { (Queen's) }\end{array}$ & 908 & 7 & $\begin{array}{c}1.44 \mathrm{e}-02 \\
\text { (13 events) }\end{array}$ \\
\hline $\begin{array}{c}\text { G48 } \\
\text { (UC Berkley) }\end{array}$ & 40200 & 45 & $\begin{array}{c}1.42 \mathrm{e}-03 \\
(57 \text { events })\end{array}$ \\
\hline
\end{tabular}

Looking at the total number of events involved, we have considerably smaller statistics 
in the case of G16K. Also, the 'live-time' and beta rates are different in the two cases and hence we cannot directly compare their USELs. However, it is worth checking if the results of the two analyses are compatible by making some approximations. If we select the candidate neutron events from both the G16K and G48 data with charge energies that are larger than the larger of the two $6 \sigma$ charge thresholds of G16K (7.5 $\mathrm{keV}$ ), we find 2 and 21 events passing the selection for G16 and G48 respectively (see figures 7.19 and 7.20 .

A crude estimate of the live-times for the G16K and G48 analysis gives 0.5 and 5 hours respectively. If all the events in the numerator of USEL are true neutron events, the corresponding neutron rates are $4.2 \pm 0.9$ per hour for G48 and $4 \pm 2$ per hour for G16K. The number of neutron candidate events is almost identical for both the G48 iZIP and HiZIP analyses. Given the surface event discrimination ability of the iZIP detector established from the data taken underground at the site of the SuperCDMS experiment in Soudan [69], all of the candidate neutron events (bulk NR band events) observed in G48 are very likely true neutron events. If they are indeed true neutron events and we assume the same neutron flux at Queen's and UC Berkley's test facilities as well as a similar detection efficiency then the results from the two analyses are compatible with an observed rate of 2 neutron events for every half hour of live-time. Although the rate calculation is not the most rigorous, it nonetheless tells us that the discrimination of the true HiZIP detector is certainly consistent with the claims made from the G48 based HiZIP analysis. 


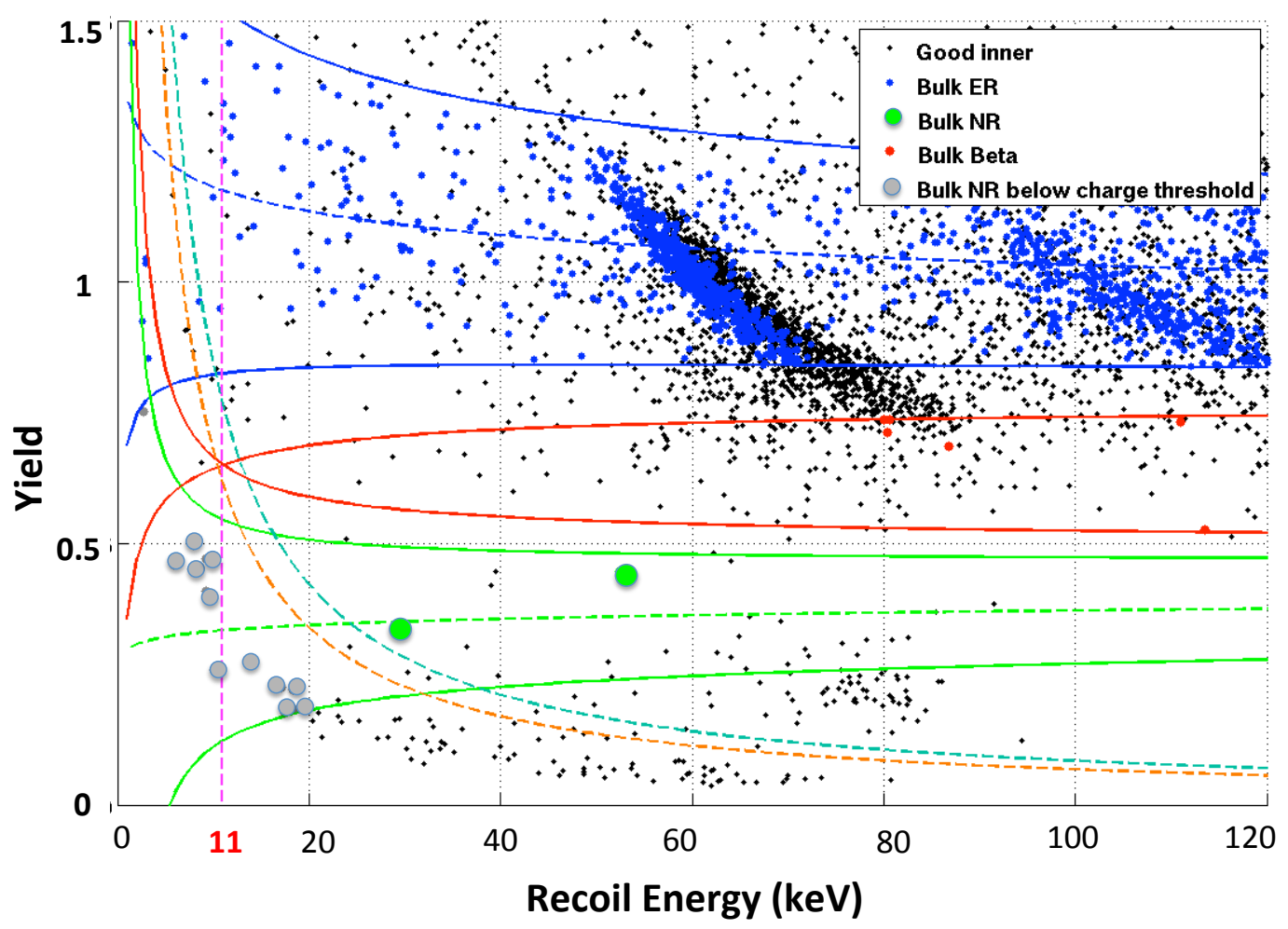

Figure 7.19: G16K surface event discrimination. The dashed vertical line (magenta) indicates the analysis threshold of $11 \mathrm{keV}$ and the dashed turquoise and dashed orange lines indicate the highest $6 \sigma$ and $4 \sigma$ charge threshold. The two green events are above the highest $6 \sigma$ charge threshold and lie in the NR band and are considered in the rate calculation. 


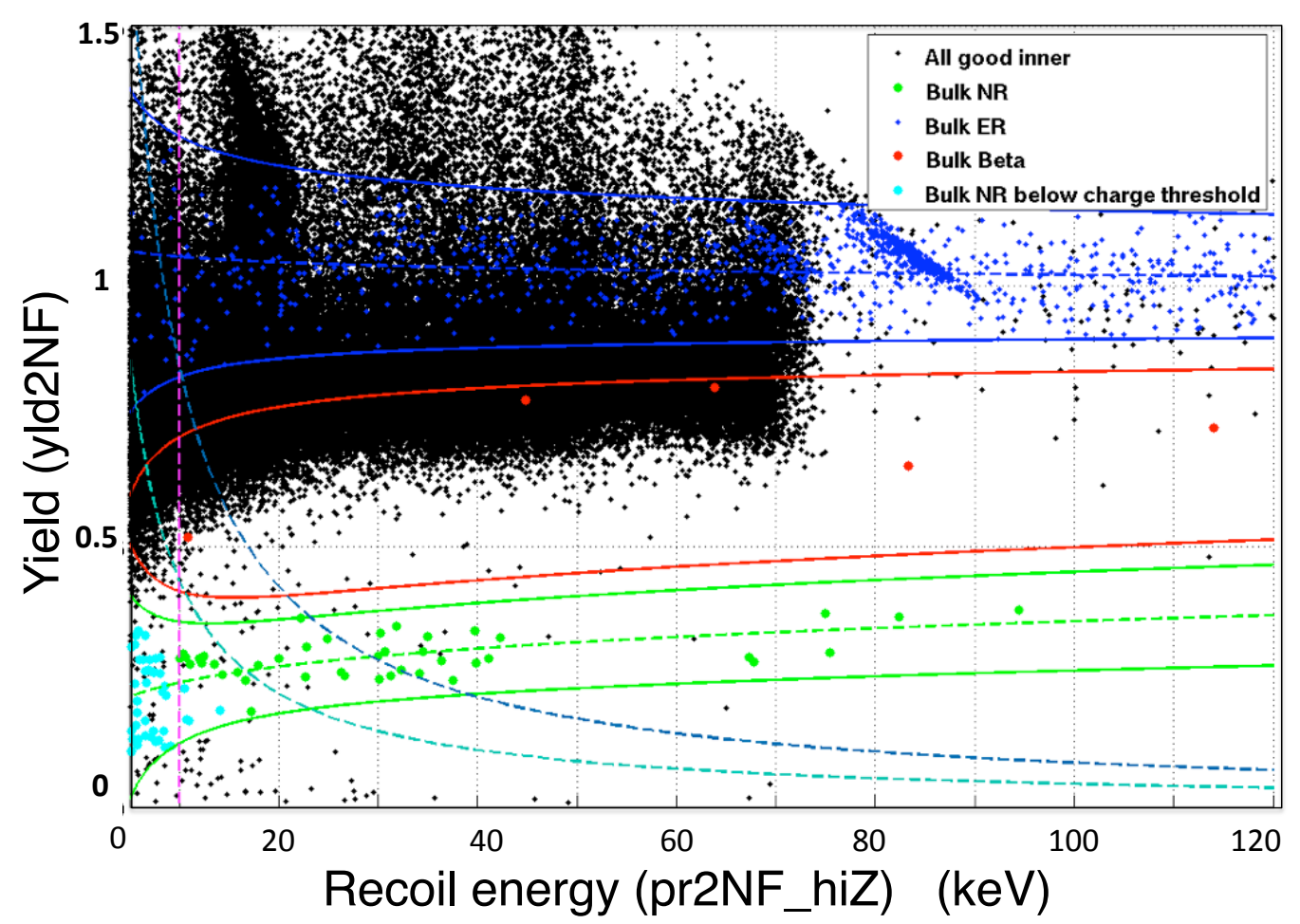

Figure 7.20: G48 surface event discrimination where the G48 detector has the same hardware and bias configuration as G16K used in this analysis. The dashed magenta line indicates the analysis threshold of $10 \mathrm{keV}$ that was used. The dashed dark green and dashed royal blue lines indicate the highest $6 \sigma$ charge thresholds of the G48 and G16K respectively. There are 21 events which are above the dashed royal blue line that lie within the NR band and are considered in the rate calculation. 


\section{Chapter 8}

\section{Conclusion}

There is a significant amount of evidence for the existence of dark matter. WIMPs are the most favoured dark matter candidate with motivations from both particle physics and cosmology. SuperCDMS aims at detecting WIMPs by measuring ionization and phonon signals from interactions in cryogenic detectors. The sensitivity of the present phase of SuperCDMS at Soudan will be limited by cosmogenic background, and hence SuperCDMS will move to SNOLAB and set up a bigger experiment. The SuperCDMS detector test facility at Queen's serves for detector testing and research and development in view of the next phase of the experiment.

The HiZIP is a modified detector concept which is explored in this work. Due to the reduced complexity in design of the HiZIP compared to an iZIP, we can expect a reduction in the complexity and quantity of data it would generate and consequently a reduction in the data analysis effort. The other major advantage of the HiZIP detector is the ability to detect MISS events (see chapter 5 for details) which may become a significant source of background in the SNOLAB phase of the experiment.

In the studies carried out in chapter 6 we estimate a $90 \%$ confidence level upper 
limit surface event leakage for a HIZIP detector of about 6 in 10,000 events, which is comparable to the value for an iZIP calculated in the same way using the same data. New phonon energy estimators based on non stationary optimal filter algorithm with a significantly reduced position dependence, made it possible to do a basic analysis without having to correct for the the position dependence explicitly.

Further, a real HiZIP device obtained by rewiring an iZIP detector (G16K) in one of the HiZIP configurations was used to take data for surface event discrimination studies at the Queen's Test Facility (QTF). This study yielded a 90\% confidence level upper limit of about 1 in 100 events which is limited by statistics. The inferences drawn from this study corroborate the findings from the G48 analysis in chapter 6 .

Studies were carried out (chapter 5) to estimate the effect of capacitive crosstalk between charge sensors in the HiZIP detector (without housing). The results from these studies indicate that the expected total noise is comparable to an iZIP detector. The new charge channel obtained from instrumenting a charge readout circuit to the interleaved phonon sensors is shown to carry useful information and behaves as expected. Due to differences in the pulse shape and pulse polarity on this channel compared to conventional charge channels, the energy reconstruction algorithms need to be modified and tuned to make the energy reconstruction precise.

All in all, these studies indicate that the HiZIP is a promising design for dark matter detection in view of a bigger experiment. Further improvements could be achieved with use of more robust phonon energy estimators which are under development. Recent results from Queen's Test Facility data with the installation of an IR shield around the detectors at the base temperature stage have improved the detector neutralization significantly, giving about 10 minutes of stable signal. This will make 
acquiring large data samples much easier in the future. It is would also be interesting to do an analysis of the kind described in chapter 6 to estimate the true surface event leakage using data from an iZIP detector underground at Soudan. This would give us a direct estimate of the surface event leakage. 


\section{Bibliography}

[1] E. Komatsu et al. Five-Year Wilkinson Microwave Anisotropy Probe Observations: Cosmological Interpretation. The Astrophysical Journal Supplement Series, 180(2):330, 2009.

[2] D. Larson et al. Seven-year Wilkinson Microwave Anisotropy Probe (WMAP) Observations: Power Spectra and WMAP-derived Parameters. The Astrophysical Journal Supplement Series, 192(2):16, 2011.

[3] C.L. Bennett et al. Nine-Year Wilkinson Microwave Anisotropy Probe (WMAP) Observations: Final Maps and Results. The Astrophysical Journal Supplement Series, 208 20, 2013.

[4] F. Zwicky. Die Rotverschiebung von extragalaktischen Nebeln. Helvetica Physica Acta: 6, 110, 1933.

[5] F. Zwicky. On the Masses of Nebulae and of Clusters of Nebulae. Astrophysical Journal: 86, 217, 1937.

[6] S. Smith. The Mass of the Virgo Cluster. Astrophysical Journal: 83, 23, 1936.

[7] Y. T Lin et al. Baryon content of massive galaxy clusters at $\mathrm{z}=0-0.6$. Astrophysical Journal Letters: 745 L3, 2012. 
[8] A. Vikhlinin et al. Chandra cluster cosmology project. II. samples and x-ray data reduction. The Astrophysical Journal 692: 1033, 2009.

[9] V. Rubin et al. On the Masses of Nebulae and of Clusters of Nebulae. Astrophysical Journal: 225, 107, 1978.

[10] V. Rubin. Seeing dark matter in the Andromeda galaxy. Physics Today, 59 (12), 8-9, 2013.

[11] E. Sheldon et al. Cross-correlation weak lensing of SDSS galaxy clusters. I. measurements. The Astrophysical Journal: 703, 2217, 2009.

[12] J. L. Tinker et al. On the Mass-to-Light Ratio of Large Scale Structure. The Astrophysical Journal: 631, 41, 2013.

[13] R. Bunker. A low-threshold analysis of data from the Cryogenic Dark Matter Search experiment. 2011. Doctoral Thesis. University of California, Santa Barbara. Available at: http://cdms. berkeley.edu/Dissertations/bunker.pdf.

[14] D. Tytler et al. Cosmological Parameters $\sigma_{8}$, the Baryon Density $\Omega_{b}$, the Vacuum Energy Density $\Omega_{\Lambda}$, the Hubble Constant and the UV Background Intensity from a Calibrated Measurement of $\mathrm{H}$ I Ly $\alpha$ Absorption at $\mathrm{z}=1.9$. The Astrophysical Journal: 617, 1, 2004.

[15] M. Markevitch et al. A textbook example of a bow shock in the merging galaxy cluster 1E 0657-56. The Astrophysical Journal: 567, L27, 2002.

[16] M. Milgrom. A modification of the Newtonian dynamics as a possible alternative to the hidden mass hypothesis. The Astrophysical Journal: 270, 365, 1983. 
[17] J.D. Bekenstein. Relativistic gravitation theory for the modified Newtonian dynamics paradigm. Physical Review D: 70, 083509, 2004.

[18] E. Pointecouteau and J. Silk. New constraints on MOND from galaxy clusters. Monthly Notices of the Royal Astronomical Society: 364 (2), 654, 2005.

[19] M. Arnaud et al. The Evolution of Galaxy Clusters Across Cosmic Time. arXiv:0902.4890, 2009.

[20] C. Afonso et al. Limits on Galactic dark matter with 5 years of EROS SMC data. Astronomy and Astrophysics: 400, 951, 2003.

[21] C. Alcock et al. MACHO Project Limits on Black Hole Dark Matter in the 130 M Range. The Astrophysical Journal: 550, L169, 2001.

[22] W. W. Ober et al. Evolution of Massive Pregalactic Stars. Astronomy and Astrophysics: 119, 61, 1983.

[23] S. D. M. White et al. The size of clusters in a neutrino-dominated universe. Astrophysical Journal: 209, 27, 1984.

[24] R. D. Peccei. and H. R. Quinn. Constraints imposed by CP conservation in the presence of pseudoparticles. Physical Review D: 16, 1791, 1977.

[25] S. Weinberg. A New Light Boson? Physical Review Letters: 40, 223, 1978.

[26] P. Sikivie . Experimental Tests of the "Invisible" Axion. Physical Review Letters: $51,1415,1983$.

[27] S.J. Asztalos et al. High resolution search for dark-matter axions. Physical Review D. 74, 012006, 2006. 
[28] L. Bergstrom. Non-Baryonic Dark Matter: Observational Evidence and Detection Methods. Reports on Progress in Physics: 63, 793, 2000.

[29] R. Gaitskell. Direct detection of dark matter. Annual Review of Nuclear and Particle Science: 54, 315, 2004.

[30] H. Goldberg. Constraint on the Photino Mass from Cosmology. Physical Review Letters: 50, 1419, 1983.

[31] J. Ellis and J. S. Hagelin. Supersymmetric relics from the Big Bang. Nuclear Physics B: 238, 453, 1983.

[32] K. Rajagopal et al. Cosmological implications of axinos. Nuclear Physics B: 358, 447, 1991.

[33] D. J. H. Chung et al. Superheavy dark matter. Physical Review D: 59, 023501, 1998.

[34] P. J. Fox et al. Missing energy signatures of dark matter at the LHC. Physical Review D: 85, 056011, 2012.

[35] O. Adriani et al. An anomalous positron abundance in cosmic rays with energies 1.5-100 GeV. Nature: 458, 607, 2008.

[36] J. Chang et al. An excess of cosmic ray electrons at energies of $300-800 \mathrm{GeV}$. Nature: 456, 362, 2009.

[37] P. J. Fox et al. Search for Photon-Linelike Signatures from Dark Matter Annihilations with H.E.S.S. Physical Review Letters: 110, 041301, 2013. 
[38] G. Zaharijas et al. Constraints on the Galactic Dark Matter signal from the Fermi-LAT measurement of the diffuse gamma-ray emission. arXiv:1304.2547, 2013.

[39] T. Tanaka et al. An Indirect Search for WIMPs in the Sun using 3109.6 days of upward-going muons in Super-Kamiokande, The Super-Kamiokande Collaboration. Astrophysical Journal: 742, 78, 2011.

[40] M. G. Aartsen et al. Search for Dark Matter Annihilations in the Sun with the 79-String IceCube Detector. Physical Review Letters: 110, 131302, 2013.

[41] A. J. Grube et al. VERITAS limits on dark matter annihilation from dwarf galaxies. AIP Conference Proceedings: 1505, 689, 2012.

[42] W. Rau. Dark matter search experiments. Physics of Particles and Nuclei, 42(4):650-660, 2011.

[43] M. W. Goodman and E. Witten. Detectability of certain dark-matter candidates. Physical Review D: 31, 3059, 1985.

[44] R. C. Cotta et al. Dark matter in the MSSM. New Journal of Physics: 11, 105026, 2009.

[45] R. Bernabei et al. DAMA/LIBRA results and perspectives. Journal of Physics: Conference Series: 375, 012002, 2012.

[46] Z. Ahmed et al. Results from a Low-Energy Analysis of the CDMS II Germanium Data. Physical Review Letters: 106, 131302, 2011. 
[47] E. Armengaud et al. Final results of the EDELWEISS-II WIMP search using a 4-kg array of cryogenic germanium detectors with interleaved electrodes. Physics Letters: B702, 329, 2011.

[48] M.G. Boulay et al. DEAP-3600 Dark Matter Search at SNOLAB. Journal of Physics: Conf. Ser. 375 012027, 2012.

[49] D.S. Akerib et al. The Large Underground Xenon (LUX) experiment. Nuclear Instruments and Methods A: 704, 111, 2013.

[50] E. Aprile et al. The XENON100 dark matter experiment. Astroparticle Physics: 35, 573, 2012.

[51] A. Davour et al. The PICASSO Dark Matter Search Project. Proc. of Identification of Dark Matter: IDM2008, 010, 2008.

[52] E. Behnke et al. Improved Limits on Spin-Dependent WIMP-Proton Interactions from a Two Liter $\mathrm{CF}_{3} \mathrm{I}$ Bubble Chamber. Physical Review Letters: 106, 021303, 2011.

[53] N. Mirabolfathi. Dark Matter Direct Detection With Low Temperature Detectors. arXiv:1308.0044, 2013.

[54] Z. Ahmed et al. Dark Matter Search Results from the CDMS II Experiment. Science: 327, 1619, 2010.

[55] E. Aprile et al. Dark matter results from 225 live days of XENON100 data. Physical Review Letters: 109, 181301, 2012. 
[56] G. Angloher et al. Results from $730 \mathrm{~kg}$ days of the CRESST-II Dark Matter Search. European Physical Journal: C72, 1971, 2012.

[57] R. Agnese et al. Dark Matter Search Results Using the Silicon Detectors of CDMS II. arXiv:1304.4279, 2013.

[58] R. Agnese et al. CDMSlite: A Search for Low-Mass WIMPs using VoltageAssisted Calorimetric Ionization Detection in the SuperCDMS Experiment. arXiv:1309.3259, 2013.

[59] D. Abrams et al. Exclusion limits on the WIMP-nucleon cross section from the Cryogenic Dark Matter Search. Physical Review D: 66, 122003, 2002.

[60] D. S. Akerib et al. Exclusion limits on the WIMP-nucleon cross section from the first run of the Cryogenic Dark Matter Search in the Soudan underground laboratory. Physical Review D: 72, 052009, 2005.

[61] Z. Ahmed et al. Search for Weakly Interacting Massive Particles with the First Five-Tower Data from the Cryogenic Dark Matter Search at the Soudan Underground Laboratory. Physical Review Letters: 102, 011301, 2009.

[62] T. Saab. Search for Weakly Interacting Massive Particles with the Cryogenic Dark Matter Search experiment. PhD thesis. Stanford University. 2002. Available at: http://cosmology.berkeley.edu/preprints/cdms/ Dissertations2/Saab_PhD_Thesis.pdf.

[63] J. Filippini. A Search for WIMP Dark Matter Using the First Five-Tower Run of the Cryogenic Dark Matter Search. PhD thesis. U C Berkeley. 2008. Available at: http://cdms.berkeley.edu/Dissertations/filippini.pdf. 
[64] J. Angle et al. First Results from the XENON10 Dark Matter Experiment at the Gran Sasso National Laboratory. Physical Review Letters: 100, 021303, 2008.

[65] P. L. Brink et al. The Cryogenic Dark Matter Search (CDMS) experiment: Results and prospects. Journal of Physics: Conference Series: 150, 012006, 2009.

[66] P. L. Brink et al. Beyond the CDMS-II dark matter search: SuperCDMS. arXiv: astro-ph/0503583, 2005.

[67] R. M. Clarke et al. Enhanced ballistic phonon production for surface events in cryogenic silicon detector. Applied Physics Letters: 76, 2958, 2000.

[68] P. L. Brink et al. First test runs of a dark-matter detector with interleaved ionization electrodes and phonon sensors for surface-event rejection. Nuclear Instruments and Methods: A559, 414, 2006.

[69] R. Agnese et al. Demonstration of Surface Electron Rejection with Interleaved Germanium Detectors for Dark Matter Search. arXiv: 1305.2405, 2013.

[70] D. S. Akerib et al. Design and performance of a modular low-radioactivity readout system for cryogenic detectors in the CDMS experiment. Nuclear Instruments and Methods: A591, 476, 2008.

[71] J. Fox. Detector Development and Test Facility Commissioning for SuperCDMS. MSc thesis. Queen's University. 2011. Available at: http://hdl.handle.net/ 1974/6537.

[72] F. Pobell. Matter and methods at low temperatures. Springer $3^{\text {rd }}$ Rev. and Extended ed., NewYork, 2007. 
[73] G. Ventura and L. Risegari. The art of cryogenics. Elsevier. Oxford, UK., 2008.

[74] C. N. Bailey. The Cryogenic Dark Matter Search: First 5-tower data and improved understanding of ionization collection. $\mathrm{PhD}$ thesis. Case Western Reserve University. 2010. Available at: http://cdms.berkeley.edu/Dissertations/ cnbailey_thesis.pdf.

[75] W. Rau. SuperCDMS internal notes: http://cdms.phy.queensu.ca/cdms _ restricted/Cryostat/Runs/Run_041/Notes/Neutralization.html, June 2013

[76] M. Pyle and B. Cabrera. SuperCDMS internal notes: http://titus. stanford.edu/cdms_restricted/detector_physics/iZIP/ebook/101022/ MISSdiscrim.html, October 2010.

[77] K. McCarthy. SuperCDMS internal notes: http://titus.stanford.edu/ cdms_restricted/kevmc/20120718_MISSevents/20120717_poscorr.html, July 2012.

[78] K. McCarthy. SuperCDMS internal notes: http://titus.stanford.edu/cdms_ restricted/detector_physics/iZIP/ebook/120523/20120523_MISSevents. html, May 2012.

[79] S. Hertel. SuperCDMS internal notes: http://titus.stanford.edu/cdms _ restricted/detector_physics/iZIP/ebook/120517/capacitances.html, May 2012. 
[80] L. Hsu. SuperCDMS internal notes: http://cdms.berkeley. edu/wiki/doku.php?id=software:cdmsbats_batroot_user_guide: optimalfilterchargex\&s []=crosstalk, August 2011.

[81] Z. Ahmed and L. Hsu. SuperCDMS internal notes: http://cdms.berkeley. edu/wiki/doku.php?id=analysis:r125:optimal_filter\&s[]=optimal\&s [] = filter, July 2010.

[82] M. Pyle and B. Serfass. SuperCDMS internal notes: http://titus. stanford.edu/cdms_restricted/mpyle1/ebooks/IZIP2/YPt_poscor_free/ Ypt_pcfree.html, October 2011.

[83] M. Pyle and B. Serfass. SuperCDMS internal notes: http://titus.stanford. edu/cdms_restricted/detector_physics/iZIP/ebook/101205/PhononCal. html, November 2010.

[84] Y. Ricci, C. Crewdson, and O. Kamaev. SuperCDMS internal notes: http://titus.stanford.edu/cdms_restricted/detector_physics/iZIP/ ebook/111130_hiZIP_reproc/note.html, November 2011.

[85] C. Crewdson. SuperCDMS internal notes: http://titus.stanford.edu/ cdms_restricted/detector_physics/iZIP/ebook/120523_Efficiencies_ and_leakage/cuts/cPChiSqNote_120523/cPChiSqNote_120523.html, May 2012.

[86] C. Martinez. SuperCDMS internal notes: http://cdms.phy.queensu.ca/cdms_ restricted/Analysis_MC/1SideiZip/notes/Efficiencies_and_leakage/ cuts/cGlitch/cGlitch.html, May 2012. 
[87] M. Pyle. Optimizing the design and analysis of cryogenic semiconductor dark matter detectors for maximum sensitivity. PhD thesis. Stanford University. 2012. Available at: http://cdms.berkeley.edu/Dissertations/mpyle1_v2.pdf.

[88] Y. Ricci, C. Crewdson, K. Page, and C. Martinez. SuperCDMS internal notes: http://titus.stanford.edu/cdms_restricted/detector_physics/iZIP/ ebook/120523_Efficiencies_and_leakage/efficiency_leakage.html, June 2012.

[89] B. Cabrera, M. Pyle, R. Moffatt, K. Sundqvist, and B. Sadoulet. Oblique propagation of electrons in crystals of germanium and silicon at sub-Kelvin temperature in low electric fields. arXiv:1004.1233, 2010.

[90] M. Pyle and B. Serfass. SuperCDMS internal notes: http://titus.stanford. edu/cdms_restricted/mpyle1/ebooks/IZIP2/qiqo_issues/IZIP2_qiqo. html, March 2012.

[91] Y. Ricci, O. Kamaev, and C. Martinez. SuperCDMS internal notes: http://titus.stanford.edu/cdms_restricted/detector_physics/iZIP/ ebook/110713_hizip_fid_cut_eff/hiZIP_fid_cut_eff_note.html, July 2011.

[92] T. Doughty. SuperCDMS internal notes: http://titus.stanford.edu/cdms_ restricted/tdoughty1/eBooks/R400/R400-Time.html, September 2010.

[93] C. Chase and K. Page. SuperCDMS internal notes: http://cdms.phy. queensu.ca/cdms_restricted/Analysis_MC/G16K_hiZIP_Analysis/G16K_ HiZIP_Notes/DTC_200513/Queess_hiZIP_DTC_200513.html, May 2013. 


\section{Appendix A}

\section{Noise Calculations}

\section{A.1 Function Definitions}

Two impedance in parallel:

$$
Z \operatorname{para}\left(Z_{1}, Z_{2}\right)=\frac{Z_{1} Z_{2}}{Z_{1}+Z_{2}}
$$

Three impedances in parallel:

$$
Z \operatorname{para} 3\left(Z_{1}, Z_{2}, Z_{3}\right)=\frac{1}{1 / Z_{1}+1 / Z_{2}+1 / Z_{3}}
$$

Two impedances in series:

$$
Z \operatorname{ser}\left(Z_{1}, Z_{2}\right)=Z_{1}+Z_{2}
$$


Magnitude of impedance squared:

$$
\operatorname{Zsquare}\left(Z_{1}\right)=Z_{1} * \bar{Z}_{2}
$$

\section{A.2 Derivation of $\mathrm{Q}_{i} \mathrm{CTF}$ and intrinsic channel noise}

In this derivation, we will first derive the voltage transfer function $\left(H_{v}(s)\right)$ of the $\mathrm{Q}_{i}$ circuit. Then we will find the voltage drop caused by various noise current sources at the gate of the $\mathrm{Q}_{i}$ amplifier and multiply them with $H_{v}(s)$ which will tell us the voltage noise spectral density at the output of the $Q_{i}$ amplifier. To factor the currents between the two circuits the impedances looking into the gate node of the amplifiers have been approximated to their respective steady state impedances.

Impedance of gate capacitance (applies to both figure 5.6 and 5.7):

$$
Z_{g}(s)=1 /\left(s C_{g}\right)
$$

Impedance of coupling capacitance (applies to both figure 5.6 and 5.7):

$$
Z_{c}(s)=1 /\left(s C_{c}\right)
$$

Impedance of feedback network (applies to both figure 5.6 and 5.7):

$$
Z_{f}(s)=\operatorname{Zpara}\left(R_{f}, 1 /\left(s C_{f}\right)\right)
$$


Net impedance of feedback network and gate capacitance in parallel (applies to both figure 5.6 and 5.7):

$$
Z_{t 1}(s)=Z \operatorname{para}\left(Z_{f}(s), 1 /\left(s C_{g}\right)\right)
$$

Net impedance of $Z_{t 1}$ and coupling capacitance in series (applies to both figure 5.6 and 5.7):

$$
Z_{t 2}(s)=Z \operatorname{ser}\left(Z_{t 1}(s), Z_{c}(s)\right)
$$

Net impedance of $Z_{t 2}$ and bias resistance in parallel (applies to both figure 5.6 and $5.7)$ :

$$
Z_{t 3}(s)=Z \operatorname{para}\left(Z_{t 2}, R_{b}\right)
$$

Net impedance of $Z_{t 3}$ and detector capacitance of $\mathrm{Q}_{o}$ in parallel (in figure 5.7):

$$
Z_{Q o C K T}(s)=Z \operatorname{para}\left(Z_{t 3}, 1 /\left(s C_{d Q o}\right)\right)
$$

Net impedance of $Z_{t 3}$ and detector capacitance of $Q_{i}$ in parallel (in figure 5.6):

$$
Z_{Q i C K T}(s)=Z \operatorname{para}\left(Z_{t 3}, 1 /\left(s C_{d Q i}\right)\right)
$$

Total impedance of $\mathrm{Q}_{o}$ seen by $\mathrm{Q}_{i}$ including $C_{Q i Q_{o}}$ (see figure 5.6):

$$
Z_{Q o}(s)=Z \operatorname{ser}\left(Z_{Q o C K T}(s), 1 /\left(s C_{Q i Q o}\right)\right)
$$


Similarly total impedance of $\mathrm{Q}_{i}$ seen by $\mathrm{Q}_{o}$ including $C_{Q^{i Q_{o}}}$ :

$$
Z_{Q i}(s)=Z \operatorname{ser}\left(Z_{Q i C K T}(s), 1 /\left(s C_{Q i Q o}\right)\right)
$$

Net impedance of $\mathrm{R}_{b}$ and $\mathrm{C}_{d Q_{o}}$ in parallel (in $\mathrm{Q}_{o}$ circuit):

$$
Z_{\text {inQo }}(s)=\operatorname{Zpara}\left(R_{b}, 1 /\left(s C_{d Q_{o}}\right)\right)
$$

Net impedance of $\mathrm{R}_{b}$ and $\mathrm{C}_{d Q i}$ in parallel (in $\mathrm{Q}_{i}$ circuit):

$$
Z_{\text {inQi }}(s)=\operatorname{Zpara}\left(R_{b}, 1 /\left(s C_{d Q i}\right)\right)
$$

Net impedance on detector side of coupling capacitor at input of $\mathrm{Q}_{o}$ seen by $\mathrm{I}_{Q_{o}}$ (see figure ):

$$
Z_{i n T o t Q_{o}}(s)=\operatorname{Zpara3}\left(Z_{Q_{i}}(s), R_{b}, 1 /\left(s C_{d Q_{o}}\right)\right)
$$

Similarly net impedance on detector side of coupling capacitor at input of $\mathrm{Q}_{i}$ seen by $\mathrm{I}_{Q i}$ :

$$
Z_{\text {inTotQi }}(s)=Z \operatorname{para3}\left(Z_{Q o}(s), R_{b}, 1 /\left(s C_{d Q i}\right)\right)
$$

Factor of current entering coupling capacitor from detector side at node 'A'. (see figure 5.6):

$$
D(s)=\frac{Z_{\text {inTotQi }}(s)}{Z \operatorname{para}\left(Z_{f}(s), Z_{g}(s)\right)+Z_{c}(s)+Z_{\text {inTotQi }}(s)}
$$


Voltage drop from $D(s)$ at the gate of the Op-Amp:

$$
G(s)=D(s) Z \operatorname{para}\left(Z_{f}(s), Z_{g}(s)\right)
$$

Net impedance across which a current entering at node 'B' would drop (see figure $5.6)$ :

$$
T(s)=Z \operatorname{para}\left(Z \operatorname{para} 3\left(R_{f}, 1 /\left(s C_{f}\right), 1 /\left(s C_{g}\right)\right),+Z_{i n T o t Q i}(s)+Z_{c}(s)\right)
$$

Reciprocal of the impedance across which a series voltage noise at the FET gate of $\mathrm{Q}_{i}$ amplifier would drop:

$$
N(s)=1 / Z \operatorname{para}\left(Z_{g}(s), Z_{\text {inTotQi }}(s)+Z_{c}(s)\right)
$$

Factor to multiply a series voltage at node ' $\mathrm{B}$ ' to obtain the voltage at output node 'O' (see figure 5.6):

$$
M(s)=N(s)\left(1 / N(s)+Z_{f}(s)\right)
$$

Amplifier open loop gain:

$$
\alpha(s)=\frac{\text { Gain }}{s G_{p}+1}
$$

Feedback factor (fraction of output voltage subtracted from gate voltage):

$$
\beta(s)=\frac{Z \operatorname{para}\left(Z_{g}(s), Z_{c}(s)+Z_{i n T o t Q i}(s)\right)}{Z \operatorname{para}\left(Z_{g}(s), Z_{c}(s)+Z_{i n T o t Q i}(s)\right)+Z_{f}(s)}
$$


Voltage transfer function for $\mathrm{Q}_{i}$ circuit:

$$
H_{v}(s)=\frac{\alpha(s)}{1+\alpha(s) \beta(s)}
$$

Current transfer function for $\mathrm{Q}_{i}$ circuit:

$$
H_{c}(s)=H_{v}(s) G(s)
$$

Johnson noise from $\mathrm{R}_{f}$ at the output of the amplifier in $\mathrm{Q}_{i}$ circuit (see figure 5.6):

$$
R_{f \text { Noise }}(s)=H_{v}(s) T(s) \sqrt{\frac{4 k T}{R_{f}}}
$$

Johnson noise from $\mathrm{R}_{b}$ at the output of the amplifier in $\mathrm{Q}_{i}$ circuit (see figure 5.6):

$$
R_{b N o i s e}(s)=H_{v}(s) G(s) \sqrt{\frac{4 k T}{R_{b}}}
$$

Voltage noise of the FET at the output of the amplifier in $\mathrm{Q}_{i}$ circuit (see figure 5.6):

$$
F \operatorname{ET}_{\text {Noise }}(s)=e_{F E T} M(s)
$$

Total intrinsic noise at the output of the amplifier in $Q_{i}$ circuit (see figure 5.6):

$$
Q_{\text {intr }}(s)=\sqrt{\left|F \operatorname{ET}_{\text {Noise }}(s)\right|^{2}+\left|R_{b N o i s e}(s)\right|^{2}+\left|R_{f \text { Noise }}(s)\right|^{2}}
$$




\section{A.3 Crosstalk from $\mathbf{Q}_{o}$ noise sources in $\mathbf{Q}_{i}$}

Factor of current entering node ' $\mathrm{P}$ ' the $\mathrm{Q}_{o}$ circuit that goes into $\mathrm{Q}_{i}$ circuit through $C_{Q i Q o}$ :

$$
M_{C T \text { factor }}(s)=\frac{Z_{\text {inQo }}(s)}{Z_{\text {inQo }}(s)+Z_{Q i}(s)}
$$

Factor of current entering node ' $\mathrm{P}$ ' the $\mathrm{Q}_{o}$ circuit that goes into $\mathrm{Q}_{i}$ circuit through $C_{Q i Q o}:$

$$
P_{C T \text { factor }}(s)=\frac{Z \operatorname{para}\left(Z_{f}(s), Z_{g}(s)\right)}{\left(Z \operatorname{para}\left(Z_{f}(s), Z_{g}(s)\right)+Z \operatorname{ser}\left(Z_{c}(s)+Z_{\text {inTotQo }}\right)\right)}
$$

Factor of current entering coupling capacitor from amplifier side of the $\mathrm{Q}_{o}$ :

$$
V_{C T \text { factor }}(s)=\frac{Z \operatorname{para}\left(Z_{f}(s), Z_{g}(s)\right)}{\left(Z \operatorname{para}\left(Z_{f}(s), Z_{g}(s)\right)+Z \operatorname{ser}\left(Z_{c}(s)+Z_{\text {inTotQo }}\right)\right)}
$$

Factor of current entering the $\mathrm{Q}_{i}$ circuit's coupling capacitor from $\mathrm{Q}_{o}$ circuit through $C_{Q i Q o}:$

$$
D_{C T \text { factor }}(s)=\frac{Z_{\text {inQi }}(s)}{Z_{p a r a}\left(Z_{f}(s), Z_{g}(s)\right)+Z_{c}(s)+Z_{\text {inQi }}(s)}
$$

Voltage drop from $D_{C T \text { factor }}(s)$ at the gate of the Op-Amp:

$$
G_{C T \text { factor }}(s)=D_{C T \text { factor }}(s) Z \operatorname{para}\left(Z_{f}(s), Z_{g}(s)\right)
$$

Reciprocal of the impedance across which a series voltage noise at the FET gate of Qo would drop: 


$$
N_{Q o C K T}(s)=1 / Z_{p a r a}\left(Z_{g}(s), Z_{\text {inTotQo }}(s)+Z_{c}(s)\right)
$$

Current transfer function for a current in 'PA' brach entering node $\mathrm{A}$ at the output of $\mathrm{Q}_{i}$ circuit:

$$
H_{i C T \text { factor }}(s)=G_{C T \text { factor }}(s) H_{v}(s)
$$

Fraction of Johnson noise current from $\mathrm{R}_{f}$ of $\mathrm{Q}_{o}$ entering node $\mathrm{A}$ into $\mathrm{Q}_{i}$ circuit:

$$
I_{\text {fbCTNoise }}(s)=P_{C T \text { factor }}(s) M_{C T \text { factor }}(s) \sqrt{\frac{4 k T}{R_{f}}}
$$

Fraction of Johnson noise current from $\mathrm{R}_{b}$ of $\mathrm{Q}_{o}$ entering node $\mathrm{A}$ into $\mathrm{Q}_{i}$ circuit:

$$
I_{r b C T N o i s e}(s)=\frac{Z_{Q o C K T}(s)}{Z_{Q o C K T}(s)+Z_{Q i}} \sqrt{\frac{4 k T}{R_{b}}}
$$

Current created by the voltage noise of FET of $\mathrm{Q}_{o}$ amplifier entering the coupling capacitor of $\mathrm{Q}_{o}$ circuit:

$$
I_{c c Q o F E T}(s)=N_{Q o C K T}(s)\left(\frac{Z_{g}(s)}{Z_{g}(s)+Z_{i n T o t Q o}(s)}\right) e_{F E T}
$$

Fraction of current created by the voltage noise of FET of $\mathrm{Q}_{o}$ amplifier entering node A into $Q_{i}$ circuit:

$$
I_{\text {fetCTNoise }}(s)=M_{C T \text { factor }}(s) I_{c c Q o F E T}(s)
$$

Voltage noise from Johnson noise of $\mathrm{R}_{f}$ of $\mathrm{Q}_{o}$ circuit measured at output of the $\mathrm{Q}_{i}$ amplifier: 


$$
V_{\text {rfCTout }}(s)=H_{i C T \text { factor }}(s) I_{f b C T \text { Noise }}(s)
$$

Voltage noise from Johnson noise of $\mathrm{R}_{b}$ of $\mathrm{Q}_{o}$ circuit measured at output of the $\mathrm{Q}_{i}$ amplifier:

$$
V_{r b C T o u t}(s)=H_{i C T \text { factor }}(s) I_{r b C T \text { Noise }}(s)
$$

Voltage noise from FET of $\mathrm{Q}_{o}$ measured at amplifier entering node $\mathrm{A}$ into $\mathrm{Q}_{i}$ circuit:

$$
V_{\text {fetCTout }}(s)=H_{\text {iCT factor }}(s) I_{c c Q o F E T}(s)
$$

Total cross coupled noise from $\mathrm{Q}_{o}$ circuit measured at the amplifier output of $\mathrm{Q}_{i}$ circuit:

$$
Q_{i C T}(s)=\sqrt{\left|V_{\text {rfCTout }}(s)\right|^{2}+\left|V_{\text {rbCTout }}(s)\right|^{2}+\left|V_{\text {fetCTout }}(s)\right|^{2}}
$$

Total at the amplifier output of $\mathrm{Q}_{i}$ circuit is the sum of intrinsic noise and the crosstalk noise:

$$
Q_{i T o t \text { Noise }}(s)=\sqrt{\left|Q_{i I n t r}(s)\right|^{2}+\left|Q_{i C T}(s)\right|^{2}}
$$




\section{A.4 Component values and constants}

The following are the values assumed in the results mentioned in chapter 5 for the above calculations.

$$
\begin{aligned}
& \mathrm{R}_{f}=40 M \Omega \\
& \mathrm{C}_{f}=1 p F \\
& R_{b}=40 M \Omega \\
& \mathrm{C}_{d} Q_{i}=57.10 p F \\
& C_{g}=75 p F \\
& C Q_{i} Q_{o}=2.811 p F \\
& C_{d} Q_{o}=24.20 p F \\
& C_{c}=300 p F \\
& G a i n=17916.475834532823 \\
& G_{p}=0.0000629 \\
& k=1.38 \times 10^{-23} \mathrm{~m}^{2} \mathrm{~kg}^{-2} \mathrm{~K}^{-1} \\
& \mathrm{~T}=0.04 \mathrm{~K} \\
& \mathrm{eFet}=0.5 \mathrm{nV} / \sqrt{\mathrm{Hz}^{-1}} \\
& \mathrm{~s}=2 \pi i f
\end{aligned}
$$

